\title{
SCATTERING IN THE ENERGY SPACE FOR THE NLS WITH VARIABLE COEFFICIENTS
}

\author{
BIAGIO CASSANO AND PIERO D'ANCONA
}

\begin{abstract}
We consider the NLS with variable coefficients in dimension $n \geq 3$ $i \partial_{t} u-L u+f(u)=0, \quad L v=\nabla^{b} \cdot\left(a(x) \nabla^{b} v\right)-c(x) v, \quad \nabla^{b}=\nabla+i b(x)$, on $\mathbb{R}^{n}$ or more generally on an exterior domain with Dirichlet boundary conditions, for a gauge invariant, defocusing nonlinearity of power type $f(u) \simeq$ $|u|^{\gamma-1} u$. We assume that $L$ is a small, long range perturbation of $\Delta$, plus a potential with a large positive part. The first main result of the paper is a bilinear smoothing (interaction Morawetz) estimate for the solution.

As an application, under the conditional assumption that Strichartz estimates are valid for the linear flow $e^{i t L}$, we prove global well posedness in the energy space for subcritical powers $\gamma<1+\frac{4}{n-2}$, and scattering provided $\gamma>1+\frac{4}{n}$. When the domain is $\mathbb{R}^{n}$, by extending the Strichartz estimates due to Tataru [32], we prove that the conditional assumption is satisfied and deduce well posedness and scattering in the energy space.
\end{abstract}

\section{INTRODUCTION}

We study the Cauchy problem in the energy space for the semilinear Schrödinger equation

$$
i \partial_{t} u-L u+f(u)=0, \quad u(0, x)=u_{0}(x)
$$

on an exterior domain $\Omega=\mathbb{R}^{n} \backslash \omega$ with $C^{1}$ boundary, in dimension $n \geq 3$, where $\omega$ is compact and possibly empty. Here $L$ is a second order elliptic operator defined on $\Omega$ with Dirichlet boundary conditions, of the form

$$
L v=\nabla^{b} \cdot\left(a(x) \nabla^{b} v\right)-c(x) v, \quad \nabla^{b}=\nabla+i b(x),
$$

where $a(x)=\left[a_{j k}(x)\right]_{j, k=1}^{n}, b(x)=\left(b_{1}(x), \ldots, b_{n}(x)\right)$ and $c(x)$ satisfy

$a, b, c$ are real valued, $a_{j k}=a_{k j}$ and $N I \geq a(x) \geq \nu I$ for some $N \geq \nu>0$.

The low dimensional cases $n \leq 2$ require substantial modifications of our techniques and will be the object of future work.

Our main results can be summarized as follows. Assume that

(i) the principal part of $L$ is a small, long range perturbation of $\Delta$;

(ii) $b, c$ have an almost critical decay, with $b$ and $c_{-}:=\max \{0,-c\}$ small;

(iii) the boundary $\partial \Omega$ is starshaped with respect to the metric induced by $a(x)$;

(iv) the nonlinearity $f(u) \simeq|u|^{\gamma-1} u$ is of power type, gauge invariant, defocusing, with $\gamma$ in the subcritical range $1 \leq \gamma<1+\frac{4}{n-2}$.

Then we prove:

(1) a virial identity for (1.1), from which we deduce a smoothing and a bilinear smoothing (interaction Morawetz) estimate for solutions of (1.1).

(2) global well posedness and scattering in the energy space for the Cauchy problem (1.1), under the black box assumption that Strichartz estimates are valid for the linear flow $e^{i t L}$; scattering requires $\gamma>1+\frac{4}{n}$.

Date: July 30, 2021.

2000 Mathematics Subject Classification. 35L70, 58J45. 
(3) in the case $\Omega=\mathbb{R}^{n}$, we extend the Strichartz estimates proved by Tataru [32] to the case of large electric potentials; hence we can drop the black box assumption and we obtain well posedness and scattering in the energy space for (1.1).

Note that for exterior domains, Strichartz estimates are known but only locally in time, see e.g. [2], [1] and the references therein. However, research on this topic is advancing rapidly, thus in the general case $\Omega \neq \mathbb{R}^{n}$ we decided to assume $a$ priori the validity of Strichartz estimates. In the case $\Omega=\mathbb{R}^{n}$ sufficiently strong results are already available and we use them to close the proof of scattering. On a related note we mention the global smoothing estimates on the exterior of polygonal domains proved in [1].

The theory of Strichartz estimates on $\mathbb{R}^{n}$ is extensive and many results are known. We mention in particular [35], [36], [36], [29] [10] for the case of electric potentials, [11] and [15] for magnetic potentials, and, for operators with fully variable coefficients, [30], [28] and [32] (see also the refences therein). Note that large perturbations in the second order terms require suitable nontrapping assumptions, which are implicit here in the assumption that $|a(x)-I|$ is sufficiently small.

Scattering theory is a important subject and the number of references is huge. For a comprehensive review of the classical theory and an extensive bibliography we refer to [7] (see also [17]). Smoothing estimates are also a classical subject, originated in [20] and [23], [24]. The bilinear version of smoothing estimates, also called interaction Morawetz estimates, was introduced as a tool in scattering theory in [8], [31] and recently adapted to Schrödinger equations with an electromagnetic potential in [9]. We mention that here we follow the simpler approach developed in [33], [6].

We conclude the introduction with a detailed exposition of our results. Here and in the rest of the paper we make frequent use of the basic properties of Lorentz spaces $L^{p, q}$, in particular precised Hölder, Young and Sobolev inequalities, for which we refer to [25].

In the following we denote by $|a(x)|$ the operator norm of the matrix $a(x)$, and we use the notations

$$
\begin{gathered}
\left|a^{\prime}\right|=\sum_{|\alpha|=1}\left|\partial^{\alpha} a(x)\right|, \quad\left|a^{\prime \prime}\right|=\sum_{|\alpha|=2}\left|\partial^{\alpha} a(x)\right|, \quad\left|a^{\prime \prime \prime}\right|=\sum_{|\alpha|=3}\left|\partial^{\alpha} a(x)\right|, \\
\left|b^{\prime}\right|=\sum_{j, k}\left|\partial_{x_{j}} b_{k}\right|, \quad\left|c^{\prime}\right|=\sum_{j}\left|\partial_{x_{j}} c\right| .
\end{gathered}
$$

1.1. The operator $L$ and its heat kernel $e^{t L}$. The results of this section are valid for all dimensions $n \geq 3$. Very mild conditions on the coefficients of $L$ are sufficient for selfadjointness: in Proposition 6.1 we prove by standard arguments that if

$$
b \in L^{n, \infty}, \quad c \in L^{\frac{n}{2}, \infty}, \quad\left\|c_{-}\right\|_{L^{\frac{n}{2}, \infty}}<\epsilon,
$$

with $\epsilon$ small enough (and $a(x) \in L^{\infty}$ ), then the operator $L$ defined on $C_{c}^{\infty}(\Omega)$ extends in the sense of forms to a selfadjoint, nonpositive operator with domain $H_{0}^{1}(\Omega) \cap H^{2}(\Omega)$. Throughout the paper, this operator will be referred to as the operator $L$ with Dirichlet boundary conditions; note that in all our results the assumptions are stronger than (1.4).

Under the additional assumption

$$
b^{2}+|\nabla \cdot b| \in L_{l o c}^{2}, \quad c \in L^{\frac{n}{2}, 1}, \quad\left\|c_{-}\right\|_{L^{\frac{n}{2}, 1}}<\epsilon
$$

with $\epsilon$ small enough, we prove in Proposition 6.2 that the heat kernel of $L$ satisfies a gaussian upper estimate of the form

$$
\left|e^{t L}(x, y)\right| \leq C^{\prime} t^{-\frac{n}{2}} e^{-\frac{|x-y|^{2}}{C t}}, \quad t>0 .
$$


In Proposition 6.3, assuming further that

$$
\|a-I\|_{L^{\infty}}+\left\||b|+\left|a^{\prime}\right|\right\|_{L^{n, \infty}}+\left\|b^{\prime}\right\|_{L^{\frac{n}{2}, \infty}}<\epsilon
$$

for $\epsilon$ small enough, using the previous bound we deduce the equivalence

$$
\left\|(-L)^{\sigma} v\right\|_{L^{p}} \simeq\left\|(-\Delta)^{\sigma} v\right\|_{L^{p}}, \quad 1<p<\frac{n}{2 \sigma}, \quad 0 \leq \sigma \leq 1 .
$$

1.2. Morawetz and interaction Morawetz estimates. From now on we restrict to the case when the operator $L$ is a suitable long range perturbation of $\Delta$ on $\Omega$; the precise conditions are the following.

Let $n \geq 3$ and assume that for some $0<\delta \leq 1$

$$
\left|a^{\prime}(x)\right|+|x|\left|a^{\prime \prime}(x)\right|+|x|^{2}\left|a^{\prime \prime \prime}(x)\right| \leq C_{a}\langle x\rangle^{-1-\delta},
$$

where $\langle x\rangle:=\left(1+|x|^{2}\right)^{1 / 2}$. Moreover, $b$ and the matrix $d b(x):=\left[\partial_{j} b_{\ell}-\partial_{\ell} b_{j}\right]_{j, \ell=1}^{n}$ satisfy

$$
b \in L^{n, \infty}, \quad|d b(x)| \leq \frac{C_{b}}{|x|^{2+\delta}+|x|^{2-\delta}} .
$$

The potential $c(x)$ satisfies

$$
-\frac{C_{-}^{2}}{|x|^{2}} \leq c(x) \leq \frac{C_{+}^{2}}{|x|^{2}}
$$

(which implies $c \in L^{\frac{n}{2}, \infty}$ ) and is repulsive with respect to the metric $a(x)$, meaning that

$$
a(x) x \cdot \nabla c(x) \leq \frac{C_{c}}{|x|\langle x\rangle^{1+\delta}} .
$$

The nonlinearity $f: \mathbb{C} \rightarrow \mathbb{C}$ is such that $f(0)=0$ and, for some $1 \leq \gamma<1+\frac{4}{n-2}$,

$$
|f(z)-f(w)| \leq(|z|+|w|)^{\gamma-1}|z-w|, \quad \text { for all } z, w \in \mathbb{C} .
$$

Note that it is easy to adapt our proofs to handle nonlinearities satisfying the more general assumption

$$
|f(z)-f(w)| \leq\left(1+|z|^{\gamma-1}+|w|^{\gamma-1}\right)|z-w| .
$$

We also assume that $f$ is gauge invariant, that is to say

$$
f(\mathbb{R}) \subseteq \mathbb{R} \text { and } f\left(e^{i \theta} z\right)=e^{i \theta} f(z) \quad \text { for all } \theta \in \mathbb{R}, z \in \mathbb{C} .
$$

Moreover, writing

we assume that $f$ is repulsive, i.e.,

$$
F(z):=\int_{0}^{|z|} f(s) d s
$$

$$
f(z) \bar{z}-2 F(z) \geq 0 \text { for all } z \in \mathbb{C} .
$$

Finally, concerning the domain $\Omega$, we assume that $\partial \Omega$ is $C^{1}$ and $a(x)$-starshaped, meaning that at all points $x \in \partial \Omega$ the exterior normal $\vec{\nu}$ to $\partial \Omega$ satisfies

$$
a(x) x \cdot \vec{\nu}(x) \leq 0 .
$$

In the following statement we use the Morrey-Campanato type norms defined by

$$
\|v\|_{\dot{X}}^{2}:=\sup _{R>0} \frac{1}{R^{2}} \int_{\Omega \cap\{|x|=R\}}|v|^{2} d S, \quad\|v\|_{\dot{Y}}^{2}:=\sup _{R>0} \frac{1}{R} \int_{\Omega \cap\{|x| \leq R\}}|v|^{2} d x .
$$

Moreover we use the notation $L_{T}^{2}=L^{2}(0, T)$ to denote integration in $t$ on the interval $[0, T]$, while $L_{T}^{p} L^{q}=L^{p}\left(0, T ; L^{q}(\Omega)\right)$ and $L^{p} L^{q}=L^{p}\left(\mathbb{R} ; L^{q}(\Omega)\right)$.

Theorem 1.1 (Smoothing). Let $n \geq 4, L$ the operator in (1.2), (1.3) with Dirichlet b.c. on the exterior domain $\Omega$, and assume (1.6), (1.7), (1.9) and (1.14). Let $u \in C\left(\mathbb{R}, H_{0}^{1}(\Omega)\right)$ be a solution of Problem (1.1). Then, if $N / \nu-1$ and the constants $C_{a}, C_{b}, C_{-}, C_{c}$ are sufficiently small, $u$ satisfies for all $T>0$ the estimate

$$
\|u\|_{\dot{X}_{x} L_{T}^{2}}^{2}+\left\|\nabla^{b} u\right\|_{\dot{Y}_{x} L_{T}^{2}}^{2}+\int_{0}^{T} \int_{\Omega} \frac{f(u) \bar{u}-2 F(u)}{|x|} d x d t \lesssim\|u(0)\|_{\dot{H}^{\frac{1}{2}}}^{2}+\|u(T)\|_{\dot{H}^{\frac{1}{2}}}^{2}
$$

with an implicit constant independent of $T$. 
Theorem 1.1 actually holds even in the case $n=3$, but we need a condition on $a(x)$ which essentially forces it to be diagonal, and this is of course too restrictive for our purposes (see (4.2) below). Thus in the 3D case we modify our approach and prove an estimate in terms of nonhomogeneous Morrey-Campanato norms

$$
\|v\|_{X}^{2}:=\sup _{R>0} \frac{1}{\langle R\rangle^{2}} \int_{\Omega \cap\{|x|=R\}}|v|^{2} d S, \quad\|v\|_{Y}^{2}:=\sup _{R>1} \frac{1}{R} \int_{\Omega \cap\{|x| \leq R\}}|v|^{2} d x .
$$

We also need some slightly stronger assumptions on the coefficients: we require

$$
|a(x)-I| \leq C_{I}\langle x\rangle^{-\delta}, \quad C_{I}<1
$$

moreover we assume

$$
b \in L^{3, \infty}, \quad|d b(x)| \leq \frac{C_{b}}{|x|^{2+\delta}+|x|}
$$

Then we have:

Theorem 1.2 (Smoothing, $n=3$ ). Let L the operator in (1.2), (1.3) with Dirichlet b.c. on the exterior domain $\Omega$, and assume (1.6), (1.16) (1.17), (1.8), (1.9), (1.11), (1.13), and (1.14). Let $u \in C\left(\mathbb{R}, H_{0}^{1}(\Omega)\right)$ be a solution of Problem (1.1). Then, if $N / \nu-1$ and the constants $C_{a}, C_{I}, C_{b}, C_{-}, C_{c}$ are sufficiently small, the solution $u$ satisfies for all $T>0$ the estimate

$$
\|u\|_{X_{x} L_{T}^{2}}^{2}+\left\|\nabla^{b} u\right\|_{Y_{x} L_{T}^{2}}^{2}+\int_{0}^{T} \int_{\Omega} \frac{f(u) \bar{u}-2 F(u)}{\langle x\rangle} d x d t \lesssim\|u(0)\|_{\dot{H}^{\frac{1}{2}}}^{2}+\|u(T)\|_{\dot{H}^{\frac{1}{2}}}^{2}
$$

with an implicit constant independent of $T$.

The previous results are a priori estimates on a global solution $u$, for which conservation of energy might not hold; this is why we state estimates (1.15),(1.18) on a finite time interval $[0, T]$ and we need the norm of $u$ both at $t=0$ and at $t=T$ at the right hand side. Note that it is possible to give explicit bounds on the smallness assumption on the coefficients, see Remark 4.1.

Remark 1.1. The proofs of Theorems 1.1 and 1.2 have a substantial overlap with the proof in [5] of resolvent estimates for the Helmholtz equation

$$
L u+z u=f, \quad z \in \mathbb{C} \backslash \mathbb{R} .
$$

One can indeed deduce estimates for the linear Schrödinger equation from the corresponding estimates for Helmholtz, via Kato's theory of smoothing [19], but with a loss in the sharpness of the estimates (see Corollary 1.3 in [5] for details; see also [3] for earlier results in a simpler setting).

Remark 1.2. Note that in (1.15) and (1.18) the space-time norms are reversed in $(x, t)$, due to the method of proof. In the hypoteses of Theorem 1.1, thanks to (1.15) and (2.7), (2.9), and in the hypoteses of Theorem 1.2, thanks to (1.18) and (2.9), (2.12), we deduce the standard weighted $L^{2}$ estimate

$\left\|\langle x\rangle^{-3 / 2-} u\right\|_{L_{T}^{2} L_{x}^{2}}^{2}+\left\|\langle x\rangle^{-1 / 2-} \nabla^{b} u\right\|_{L_{T}^{2} L_{x}^{2}}^{2}+\int_{0}^{T} \int_{\Omega} \frac{f(u) \bar{u}-2 F(u)}{|x|} d x d t \lesssim\|u(0)\|_{\dot{H}^{\frac{1}{2}}}^{2}+\|u(T)\|_{\dot{H}^{\frac{1}{2}}}^{2}$.

By (2.16) we can replace $\nabla^{b}$ with $\nabla$ at the left hand side, obtaining

$$
\left\|\langle x\rangle^{-3 / 2-} u\right\|_{L_{T}^{2} L_{x}^{2}}^{2}+\left\|\langle x\rangle^{-1 / 2-} \nabla u\right\|_{L_{T}^{2} L_{x}^{2}}^{2} \lesssim\|u(0)\|_{\dot{H}^{\frac{1}{2}}}^{2}+\|u(T)\|_{\dot{H}^{\frac{1}{2}}}^{2} \cdot
$$

If the assumptions on $b, c$ are slightly stronger so that the heat kernel $e^{t L}$ satisfies an upper gaussian bound, we can apply the techniques in [4] to obtain a further estimate of weighted $L^{2}$ tipe. In the next Corollary we assume $\Omega=\mathbb{R}^{n}$ to keep the proof simple but this would not be necessary. 
Corollary 1.3. Let $n \geq 3, \Omega=\mathbb{R}^{n}$, let $L$ be as in Theorem 1.1 or as in Theorem 1.2, and assume that

$$
b^{2}+|\nabla \cdot b| \in L_{l o c}^{2}, \quad c \in L^{\frac{n}{2}, 1}, \quad\left\|c_{-}\right\|_{L^{\frac{n}{2}, 1}}<\epsilon .
$$

Then for $\epsilon$ small enough the flow $e^{i t L}$ satisfies the estimate

$$
\left\|\langle x\rangle^{-1-} e^{i t L} u_{0}\right\|_{L_{t}^{2} L_{x}^{2}} \lesssim\left\|u_{0}\right\|_{L^{2}} .
$$

The next results are bilinear smoothing (interaction Morawetz) estimates for equation (1.1), which are the crucial tool in the proof of scattering. Note that the assumptions are essentially the same as in Theorems 1.1, 1.2, and the constant $C_{c^{\prime}}$ may be large.

Theorem 1.4 (Bilinear smoothing, $n \geq 4$ ). Let $n \geq 4$ and let $\Omega, L$ be as in Theorem 1.1. In addition, assume that

$$
|x|^{2}|\nabla c| \leq C_{c^{\prime}}\langle x\rangle^{-1-\delta}
$$

Let $u \in C\left(\mathbb{R}, H_{0}^{1}(\Omega)\right)$ be a solution of (1.1). Then, if the constants $C_{a}, C_{b}, C_{-}, C_{c}$ and $N / \nu-1$ are small enough, $u$ satisfies the estimate

$$
\int_{0}^{T} \int_{\Omega \times \Omega} \frac{|u(t, x)|^{2}|u(t, y)|^{2}}{|x-y|^{3}} d x d y d t \lesssim\|u(0)\|_{L^{2}}^{2}\left[\|u(0)\|_{\dot{H}^{\frac{1}{2}}}+\|u(T)\|_{\dot{H}^{\frac{1}{2}}}\right]^{2} .
$$

Theorem 1.5 (Bilinear smoothing, $n=3$ ). Let $n=3$ and let $\Omega, L$ be as in Theorem 1.2. In addition, assume (1.22). Let $u \in C\left(\mathbb{R}, H_{0}^{1}(\Omega)\right)$ be a solution of (1.1). Then, if the constants $C_{a}, C_{I}, C_{b}, C_{-}, C_{c}$ and $N / \nu-1$ are small enough, $u$ satisfies the estimate

$$
\|u\|_{L^{4}\left(0, T ; L^{4}(\Omega)\right)}^{4} \lesssim\|u(0)\|_{L^{2}}^{2}\left[\|u(0)\|_{\dot{H}^{\frac{1}{2}}}+\|u(T)\|_{\dot{H}^{\frac{1}{2}}}\right]^{2}
$$

1.3. Global existence and scattering. The proof of well posedness and scattering for (1.1) in the energy space relies in an essential way on Strichartz estimates for the linear flow $e^{i t L}$. As mentioned above, these are known in the case $\Omega=\mathbb{R}^{n}$ under various assumptions on the coefficients, while the results for exterior domains are far from complete. For this reason we decided to state our main results by assuming the validity of Strichartz estimates in a black box form, and then specialize them to some situations where Strichartz estimates are already available. Recalling that an admissible (non endpoint) couple is a couple of indices $(p, q)$ with $2<p \leq \infty$ and $2 / p+n / q=n / 2$, our black box assumption has the following form:

Assumption (S). The Schrödinger flow $e^{i t L}$ satisfies the Strichartz estimates

$$
\left\|e^{i t L} u_{0}\right\|_{L^{p_{1} L^{q_{1}}}} \lesssim\left\|u_{0}\right\|_{L^{2}}, \quad\left\|\int_{0}^{t} e^{i(t-s) L} F d s\right\|_{L^{p_{1} L^{q_{1}}}} \lesssim\|F\|_{L^{p_{2}^{\prime} L^{q_{2}^{\prime}}}}
$$

for all admissible couples $\left(p_{j}, q_{j}\right)$, while the derivative of the flow $\nabla e^{i t L}$ satisfies

$$
\left\|\nabla e^{i t L} u_{0}\right\|_{L^{p_{1}} L^{q_{1}}} \lesssim\left\|\nabla u_{0}\right\|_{L^{2}}, \quad\left\|\nabla \int_{0}^{t} e^{i(t-s) L} F d s\right\|_{L^{p_{1}} L^{q_{1}}} \lesssim\|\nabla F\|_{L^{p_{2}^{\prime} L^{q_{2}^{\prime}}}}
$$

for admissible couples $\left(p_{j}, q_{j}\right)$ such that $q_{1}<n$.

Note that it is not trivial to deduce (1.26) from (1.25): indeed, for this step one needs the equivalence of norms

$$
\left\|(-L)^{\frac{1}{2}} v\right\|_{L^{q}} \simeq\|\nabla v\|_{L^{q}}
$$

with $q$ in the appropriate range. Under fairly general assumptions on $L$, we are able to prove this equivalence for all $1<q<n$ (see (1.5)), and this is the reason for the restriction on $q_{1}$ in $(\mathrm{S})$.

Using Assumption ( $\mathrm{S}$ ) we can prove local well posedness in the energy space, and global well posedness provided the nonlinearity is defocusing, i.e.,

$$
F(r)=\int_{0}^{r} f(s) d s \geq 0 \text { for } s \in \mathbb{R}
$$


(this is the content of Proposition 7.1 and Theorem 7.2):

Theorem 1.6. Let $n \geq 3$, let $\Omega=\mathbb{R}^{n} \backslash \omega$ be an exterior domain with compact and possibly empty $C^{1}$ boundary, let $L$ be the selfadjoint operator with Dirichlet b.c. defined by (1.2), (1.3), (1.4), and assume (S) holds.

(i) (Local existence in $\left.H^{1}\right)$. If $f \in C^{1}(\mathbb{C}, \mathbb{C})$ satisfies $f(0)=0$ and $\mid f(z)-$ $f(w)\left|\lesssim(|z|+|w|)^{\gamma-1}\right| z-w \mid$ for some $1 \leq \gamma<1+\frac{4}{n-2}$, then for all $u_{0} \in$ $H_{0}^{1}(\Omega)$ there exists $T=T\left(\left\|u_{0}\right\|_{H^{1}}\right)$ and a unique solution $u \in C\left([-T, T] ; H_{0}^{1}(\Omega)\right)$.

(ii) (Global existence in $\left.H^{1}\right)$. Assume in addition that $b^{2}+|\nabla \cdot b| \in L_{l o c}^{2}$, $c \in L^{\frac{n}{2}, 1}$,

$$
\|a-I\|_{L^{\infty}}+\left\||b|+\left|a^{\prime}\right|\right\|_{L^{n, \infty}}+\left\|b^{\prime}\right\|_{L^{\frac{n}{2}, \infty}}+\left\|c_{-}\right\|_{L^{\frac{n}{2}, 1}}<\epsilon
$$

for $\epsilon$ small enough, and that $f(u)$ is gauge invariant (1.11) and defocusing (1.27). Then for all initial data $u_{0} \in H_{0}^{1}(\Omega)$ problem (7.1) has a unique global solution $u \in C \cap L^{\infty}\left(\mathbb{R} ; H_{0}^{1}(\Omega)\right)$. The solution has constant energy for all $t \in \mathbb{R}$ :

$$
E(t)=\frac{1}{2} \int_{\Omega} a(x) \nabla^{b} u \cdot \overline{\nabla^{b} u} d x+\frac{1}{2} \int_{\Omega} c(x)|u|^{2} d x+\int_{\Omega} F(u) d x \equiv E(0) .
$$

Combining the global existence result with the bilinear smoothing estimate in Theorems 1.4 and 1.5, we obtain the main results of this paper. Note that a power nonlinearity $f(u)=|u|^{\gamma-1} u$ with $1+\frac{4}{n}<\gamma<1+\frac{4}{n-2}$ satisfies all conditions of the following Theorems:

Theorem 1.7 (Scattering on $\Omega$, under $(\mathrm{S})$ ). Let $n \geq 3, \Omega=\mathbb{R}^{n} \backslash \omega$ an exterior domain with $C^{1}$ compact and possibly empty boundary satisfying (1.14), L the operator (1.2) with Dirichlet b.c. on $\Omega$. Assume a,b,c satisfy, for some $\epsilon, C>0$, $\delta \in(0,1]$

$$
|x| a(x) x \cdot \nabla c<\epsilon\langle x\rangle^{-1-\delta}, \quad|x||c|+|x|^{2}\left|c^{\prime}\right|<C\langle x\rangle^{-1-\delta},
$$

and in addition

$$
\begin{array}{ll}
\|a-I\|_{L^{\infty}}+|x|^{2} c_{-}+\left\|c_{-}\right\|_{L^{\frac{n}{2}, 1}}<\epsilon, \quad|x||b|+|x|^{2}\left|b^{\prime}\right|<\epsilon|x|^{\delta}\langle x\rangle^{-2 \delta}, & \text { if } n \geq 4 \\
\langle x\rangle^{\delta}\|a-I\|_{L^{\infty}}+\langle x\rangle^{2} c_{-}+\left\|c_{-}\right\|_{L^{\frac{n}{2}, 1}}<\epsilon, \quad|x||b|+|x|\langle x\rangle^{1+\delta}\left|b^{\prime}\right|<\epsilon, & \text { if } n=3 .
\end{array}
$$

Finally $\left|a^{\prime}\right|+|x|\left|a^{\prime \prime}\right|+|x|^{2}\left|a^{\prime \prime \prime}\right|<\epsilon\langle x\rangle^{-1-\delta}$, and $f: \mathbb{C} \rightarrow \mathbb{C}$ is gauge invariant (1.11), repulsive (1.13), defocusing (1.27) and satisfies $f(0)=0,|f(z)-f(w)| \lesssim$ $(|z|+|w|)^{\gamma-1}|z-w|$ for some $1+\frac{4}{n}<\gamma<1+\frac{4}{n-2}$. Then if $(S)$ holds and $\epsilon$ is small enough we have:

(i) (Existence of wave operators) For every $u_{+} \in H_{0}^{1}(\Omega)$ there exists a unique $u_{0} \in H_{0}^{1}(\Omega)$ such that the global solution $u(t)$ to (1.1) satisfies $\| e^{-i t L} u_{+}-$ $u(t) \|_{H^{1}} \rightarrow 0$ as $t \rightarrow+\infty$. An analogous result holds for $t \rightarrow-\infty$.

(ii) (Asymptotic completeness) For every $u_{0} \in H_{0}^{1}(\Omega)$ there exists a unique $u_{+} \in H_{0}^{1}(\Omega)$ such that the global solution $u(t)$ to $(1.1)$ satisfies $\| e^{-i t L} u_{+}-$ $u(t) \|_{H^{1}} \rightarrow 0$ as $t \rightarrow+\infty$. An analogous result holds for $t \rightarrow-\infty$.

When $\Omega=\mathbb{R}^{n}$, Strichartz estimates for $e^{i t L}$ were proved by Tataru [32] in the case $L$ is a small, long range perturbations of $\Delta$. In Theorems $8.1-8.2$ we adapt the result in [32] to our situation, and in particular, combining it with the smoothing estimate (1.15), we extend Strichartz estimates to potentials $c(x)$ with a large positive part. In addition we deduce the necessary estimates also for the derivative of the flow $\nabla e^{i t L}$ (Corollary 8.3). As a consequence, Assumption (S) is satisfied and we obtain the final result of the paper: 
Theorem 1.8 (Scattering on $\mathbb{R}^{n}$ ). Let $n \geq 3$, assume $a, b, c$ satisfy $c \in L_{l o c}^{n}$ and

$$
\begin{gathered}
|a-I|+\langle x\rangle\left(\left|a^{\prime}\right|+|b|\right)+\langle x\rangle^{2}\left(\left|a^{\prime \prime}\right|+\left|b^{\prime}\right|\right)+\langle x\rangle^{3}\left|a^{\prime \prime \prime}\right|<\epsilon\langle x\rangle^{-\delta}, \\
|x|\langle x\rangle a(x) x \cdot \nabla c<\epsilon\langle x\rangle^{-\delta}, \quad\left\|c_{-}\right\|_{L^{\frac{n}{2}, 1}}<\epsilon, \quad|x||c|+|x|^{2}\left|c^{\prime}\right|<C\langle x\rangle^{-1-\delta} . \\
|x|^{2} c_{-}<\epsilon, \quad \text { if } n \geq 4, \quad\langle x\rangle^{2} c_{-}<\epsilon, \quad \text { if } n=3,
\end{gathered}
$$

for some $C>0, \delta \in(0,1]$ and some $\epsilon$ small enough, and let $L$ be the selfadjoint operator defined by (1.2)-(1.3) on $\mathbb{R}^{n}$. Finally, assume $f: \mathbb{C} \rightarrow \mathbb{C}$ is gauge invariant (1.11), repulsive (1.13), defocusing (1.27) and satisfies $f(0)=0,|f(z)-f(w)| \lesssim$ $(|z|+|w|)^{\gamma-1}|z-w|$ for some $1+\frac{4}{n}<\gamma<1+\frac{4}{n-2}$.

Then the conclusions (i), (ii) of Theorem 1.7 are valid.

\section{Notations And ElEmentaRy iDEntities}

Using the convention of implicit summation over repeated indices, we define the operators

$$
A^{b} v:=\nabla^{b} \cdot\left(a(x) \nabla^{b} v\right)=\partial_{j}^{b}\left(a_{j k}(x) \partial_{k}^{b} v\right), \quad A v:=\nabla \cdot(a(x) \nabla v)=\partial_{j}\left(a_{j k}(x) \partial_{k} v\right)
$$

so that $L=A^{b}-c$. The quadratic form associated with $A$ is given by

$$
a(w, z):=a_{j k}(x) w_{k} \bar{z}_{j}
$$

We shall use the notations

$$
\begin{gathered}
\widehat{x}=\frac{x}{|x|}=\left(\widehat{x}_{1}, \ldots, \widehat{x}_{n}\right), \quad \widehat{x}_{j}=\frac{x_{j}}{|x|}, \\
\widehat{a}(x)=a_{\ell m}(x) \widehat{x}_{\ell} \widehat{x}_{m}, \quad \bar{a}(x)=\operatorname{trace} a(x)=a_{m m}(x) .
\end{gathered}
$$

Since $a(x)$ is positive definite, we have

$$
0 \leq \widehat{a}=a \widehat{x} \cdot \widehat{x} \leq|a \widehat{x}| \leq \bar{a} .
$$

Indices after a semicolon refer to partial derivatives:

$$
a_{j k ; \ell}:=\partial_{\ell} a_{j k}, \quad a_{j k ; \ell m}:=\partial_{\ell} \partial_{m} a_{j k}, \quad a_{j k ; \ell m p}:=\partial_{\ell} \partial_{m} \partial_{p} a_{j k} .
$$

Notice the formulas

$$
\begin{gathered}
\partial_{k}\left(\widehat{x}_{\ell}\right)=|x|^{-1}\left[\delta_{k \ell}-\widehat{x}_{k} \widehat{x}_{\ell}\right], \\
\partial_{k}\left(\widehat{x}_{\ell} \widehat{x}_{m}\right)=|x|^{-1}\left[\delta_{k \ell} \widehat{x}_{m}+\delta_{k m} \widehat{x}_{\ell}-2 \widehat{x}_{k} \widehat{x}_{\ell} \widehat{x}_{m}\right], \\
\partial_{j} \partial_{k}\left(\widehat{x}_{\ell} \widehat{x}_{m}\right)=\frac{1}{|x|^{2}}\left[\delta_{k \ell} \delta_{j m}+\delta_{k m} \delta_{j \ell}+8 \widehat{x}_{j} \widehat{x}_{k} \widehat{x}_{\ell} \widehat{x}_{m}\right. \\
\left.-2 \delta_{k \ell} \widehat{x}_{j} \widehat{x}_{m}-2 \delta_{k m} \widehat{x}_{j} \widehat{x}_{\ell}-2 \delta_{j k} \widehat{x}_{\ell} \widehat{x}_{m}-2 \delta_{j \ell} \widehat{x}_{k} \widehat{x}_{m}-2 \delta_{j m} \widehat{x}_{k} \widehat{x}_{\ell}\right]
\end{gathered}
$$

which imply

and

$$
a_{j k} a_{\ell m} \widehat{x}_{j} \partial_{k}\left(\widehat{x}_{\ell} \widehat{x}_{m}\right)=2|x|^{-1}\left[|a \widehat{x}|^{2}-\widehat{a}^{2}\right],
$$

$$
a_{j k} a_{\ell m} \partial_{j} \partial_{k}\left(\widehat{x}_{\ell} \widehat{x}_{m}\right)=\frac{2}{|x|^{2}}\left[a_{\ell m} a_{\ell m}-4\left(|a \widehat{x}|^{2}-\widehat{a}^{2}\right)-\bar{a} \widehat{a}\right] .
$$

Using the previous identities, we see that for any radial function $\psi(x)=\psi(|x|)$ we can write

$$
A \psi(x)=\partial_{\ell}\left(a_{\ell m} \widehat{x}_{m} \psi^{\prime}\right)=\widehat{a} \psi^{\prime \prime}+\frac{\bar{a}-\widehat{a}}{|x|} \psi^{\prime}+a_{\ell m ; \ell} \widehat{x}_{m} \psi^{\prime}
$$

where $\psi^{\prime}$ denotes the derivative of $\psi(r)$ with respect to the radial variable.

We now give the definitions of the Morrey-Campanato type norms $\dot{X}, \dot{Y}, X, Y$ and recall some relations between them and usual weighted $L^{2}$ norms.

For an open subset $\Omega \subseteq \mathbb{R}^{n}, n \geq 2$, we use the notations

$$
\begin{gathered}
\Omega_{=R}=\Omega \cap\{x:|x|=R\}, \quad \Omega_{\leq R}=\Omega \cap\{x:|x| \leq R\}, \quad \Omega_{\geq R}=\Omega \cap\{x:|x| \geq R\}, \\
\Omega_{R_{1} \leq|x| \leq R_{2}}=\Omega \cap\left\{x: R_{1} \leq|x| \leq R_{2}\right\} .
\end{gathered}
$$


The homogeneous and inhomogeneous norms $\dot{X}$ and $X$ of a function $v: \Omega \rightarrow \mathbb{C}$ are defined as

$$
\|v\|_{\dot{X}}^{2}:=\sup _{R>0} \frac{1}{R^{2}} \int_{\Omega_{=R}}|v|^{2} d S, \quad\|v\|_{X}^{2}:=\sup _{R>0} \frac{1}{\langle R\rangle^{2}} \int_{\Omega_{=R}}|v|^{2} d S
$$

where $d S$ is the surface measure on $\Omega_{=R}$ and $\langle R\rangle=\sqrt{1+R^{2}}$. We shall also need proper Morrey-Campanato spaces, both in the homogeneous version $\dot{Y}$ and in the non homogenous version $Y$; their norms are defined as

$$
\|v\|_{\dot{Y}}^{2}:=\sup _{R>0} \frac{1}{R} \int_{\Omega_{\leq R}}|v|^{2} d x, \quad\|v\|_{Y}^{2}:=\sup _{R>0} \frac{1}{\langle R\rangle} \int_{\Omega_{\leq R}}|v|^{2} d x .
$$

The following equivalence is easy to prove:

$$
\|v\|_{Y}^{2} \leq \sup _{R \geq 1} \frac{1}{R} \int_{\Omega_{\leq R}}|v|^{2} \leq \sqrt{2}\|v\|_{Y}^{2} .
$$

The following Lemmas collect a few estimates to be used in the rest of the paper, which follow immediately from the definitions (proofs are straightforward, and full details can be found in [5]).

Lemma 2.1. For any $v \in C^{\infty}\left(\mathbb{R}^{n}\right)$,

$$
\begin{aligned}
& \left\||x|^{-1} v\right\|_{\dot{Y}} \leq\|v\|_{\dot{X}}, \quad\left\|\langle x\rangle^{-1} v\right\|_{Y} \leq\|v\|_{X}, \\
& \sup _{R>0} \int_{\Omega_{\geq R}} \frac{R^{n-1}}{|x|^{n+2}}|v|^{2} d x \leq \frac{1}{n-1}\|v\|_{\dot{X}}^{2}, \quad \sup _{R>1} \int_{\Omega_{\geq R}} \frac{R^{n-1}}{|x|^{n+2}}|v|^{2} d x \leq \frac{2}{n-1}\|v\|_{X}^{2} .
\end{aligned}
$$

Lemma 2.2. For any $0<\delta<1$ and $v \in C^{\infty}\left(\mathbb{R}^{n}\right)$,

$$
\begin{gathered}
\int_{\Omega} \frac{|v|^{2}}{|x|^{2}\langle x\rangle^{1+\delta}} \leq 2 \delta^{-1}\|v\|_{\dot{X}}^{2}, \\
\int_{\Omega_{\geq 1}} \frac{|v|^{2}}{|x|^{3}\langle x\rangle^{\delta}} \leq \int_{\Omega_{\geq 1}} \frac{|v|^{2}}{|x|^{3+\delta}} \leq 2 \delta^{-1}\|v\|_{X}^{2}, \\
\int_{\Omega} \frac{|v|^{2}}{\langle x\rangle^{1+\delta}} \leq 8 \delta^{-1}\|v\|_{Y}^{2} \leq 8 \delta^{-1}\|v\|_{\dot{Y}}^{2} .
\end{gathered}
$$

Lemma 2.3. For any $R>0,0<\delta<1$ and $v, w \in C^{\infty}\left(\mathbb{R}^{n}\right)$,

$$
\int_{\Omega_{\leq 1}} \frac{|v w|}{|x|^{2-\delta}}+\int_{\Omega_{\geq 1}} \frac{|v w|}{|x|^{2+\delta}} \leq 9 \delta^{-1}\|v\|_{\dot{X}}\|w\|_{\dot{Y}}
$$

In the following Lemma we prove some magnetic Hardy type inequalities, which require $n \geq 3$, expressed in terms of the nonhomogeneous $X, Y$ norms (compare (2.11) with Theorem A.1 in [16]):

Lemma 2.4. Let $n \geq 3$ and assume $b(x)=\left(b_{1}(x), \ldots, b_{n}(x)\right)$ is continuous up to the boundary of $\Omega$ with values in $\mathbb{R}^{n}$. For any $0<\delta<1, y \in \Omega$ and $v \in C_{c}^{\infty}(\Omega)$, we have:

$$
\begin{gathered}
\left\||x-y|^{-1} v\right\|_{L^{2}(\Omega)} \leq \frac{2}{n-2}\left\|\nabla^{b} v\right\|_{L^{2}(\Omega)}, \\
\left\||x|^{-1} v\right\|_{Y}^{2} \leq 6\left\|\nabla^{b} v\right\|_{Y}^{2}+3\|v\|_{X}^{2}, \\
\int_{\Omega_{\leq 1}} \frac{\left|\nabla^{b} v \|\right| v \mid}{|x|} d x+\int_{\Omega_{\geq 1}} \frac{\left|\nabla^{b} v \|\right| v \mid}{|x|^{2+\delta}} d x \leq 9 \delta^{-1}\left(\left\|\nabla^{b} v\right\|_{Y}^{2}+\|v\|_{X}^{2}\right), \\
\|v\|_{X} \leq 4 \sup _{R>1} R^{-2} \int_{\Omega_{=R}}|v|^{2} d S+13\left\|\nabla^{b} v\right\|_{Y}^{2} .
\end{gathered}
$$

Proof. We give the complete proof of (2.11); the remaining inequalities are proved in [5]. Integrating on $\Omega$ the identity

$$
\nabla \cdot\left\{\frac{x-y}{|x-y|^{2}}|v|^{2}\right\}=\Re\left[2 c(x) \overline{\nabla^{b} f(x)} \frac{x-y}{|x-y|^{2}}\right]+(n-2) \frac{|c(x)|^{2}}{|x-y|^{2}}
$$

and noticing that boundary term vanishes, we get

$$
\frac{n-2}{2} \int_{\Omega} \frac{|f(x)|^{2}}{|x-y|^{2}} d x \leq \Re \int_{\Omega} \frac{(x-y) f(x)}{|x-y|^{2}} \overline{\nabla^{b} f(x)} d x \leq\left(\int_{\Omega} \frac{|f(x)|^{2}}{|x-y|^{2}}\right)^{\frac{1}{2}}\left(\int_{\Omega}\left|\nabla^{b} f(x)\right|^{2} d x\right)^{\frac{1}{2}} .
$$


By a density argument, it is clear that the previous estimates are valid not only for smooth functions but also for functions in $D(L)=H_{0}^{1}(\Omega) \cap H^{2}(\Omega)$.

We conclude this section with some additional properties of the magnetic norms.

Lemma 2.5. Let $n \geq 3$. If $b \in L^{n, \infty}(\Omega)$, the following equivalence holds:

$$
\left\|\nabla^{b} v\right\|_{L^{2}(\Omega)} \simeq\|\nabla v\|_{L^{2}(\Omega)}
$$

Moreover, for $s>0$ we have

$$
\left\|\langle x\rangle^{-s} \nabla^{b} v\right\|_{L^{2}(\Omega)}+\left\|\langle x\rangle^{-s-1} v\right\|_{L^{2}(\Omega)} \simeq\left\|\langle x\rangle^{-s} \nabla v\right\|_{L^{2}(\Omega)}+\left\|\langle x\rangle^{-s-1} v\right\|_{L^{2}(\Omega)} .
$$

Proof. By Hölder inequality and Sobolev embedding in Lorentz spaces, we can write $\left\|\nabla^{b} v\right\|_{L^{2}} \leq\|\nabla v\|_{L^{2}}+\|b v\|_{L^{2}} \leq\|\nabla v\|_{L^{2}}+\|b\|_{L^{n, \infty}}\|v\|_{L^{\frac{2 n}{n-2}, 2}} \lesssim\left(1+\|b\|_{L^{n, \infty}}\right)\|\nabla v\|_{L^{2}}$.

Conversely, writing $\nabla=\nabla^{b}-i b$, we have

$$
\|\nabla v\|_{L^{2}} \leq\left\|\nabla^{b} v\right\|_{L^{2}}+\|b v\|_{L^{2}} \lesssim\left\|\nabla^{b} v\right\|_{L^{2}}+\|b\|_{L^{n, \infty}}\|v\|_{L^{\frac{2 n}{n-2}, 2}} .
$$

Recall now the pointwise diamagnetic inequality

$$
|\nabla| v|| \leq\left|\nabla^{b} v\right|
$$

which is true for $b \in L_{l o c}^{2}$. Thus, again by Sobolev-Lorentz embedding,

$$
\|v\|_{L^{\frac{2 n}{n-2}, 2}} \lesssim\|\nabla|v|\|_{L^{2}} \leq\left\|\nabla^{b} v\right\|_{L^{2}}
$$

and we obtain (2.15). Next we can write

$$
\left\|\langle x\rangle^{-s} \nabla v\right\|_{L^{2}}+\left\|\langle x\rangle^{-s-1} v\right\|_{L^{2}} \simeq\left\|\nabla\left(\langle x\rangle^{-s} v\right)\right\|_{L^{2}}+\left\|\langle x\rangle^{-s-1} v\right\|_{L^{2}}
$$

and

$$
\left\|\langle x\rangle^{-s} \nabla^{b} v\right\|_{L^{2}}+\left\|\langle x\rangle^{-s-1} v\right\|_{L^{2}} \simeq\left\|\nabla^{b}\left(\langle x\rangle^{-s} v\right)\right\|_{L^{2}}+\left\|\langle x\rangle^{-s-1} v\right\|_{L^{2}}
$$

which, together with (2.15), imply (2.16).

Lemma 2.6. Let $n \geq 3$ and consider the operator $L=A^{b}-c$ with Dirichlet b.c. on $\Omega$, under assumptions (1.3), (1.6), (1.7), (1.9) and (1.14). If the constant $C_{-}$is sufficiently small, the operator $L$ is selfadjoint and nonpositive. If in addition $b \in L^{n, \infty}(\Omega)$ then for all $0 \leq s \leq 1$ we have the equivalence

$$
\left\|(-L)^{\frac{s}{2}} v\right\|_{L^{2}(\Omega)} \simeq\|v\|_{\dot{H}^{s}(\Omega)} .
$$

Proof. Selfadjointness and positivity are standard, and actually hold under less restrictive assumptions on the coefficients (see Proposition 6.3 below for a more general result). Next, (2.18) is trivial for $s=0$, while for $s=1$ we have

$$
\left\|(-L)^{\frac{1}{2}} v\right\|_{L^{2}}^{2}=(-L v, v)_{L^{2}(\Omega)}=a\left(\nabla^{b} v, \nabla^{b} v\right)+\int_{\Omega} c|v|^{2} d x
$$

which implies, using (2.15),

$$
\left\|(-L)^{\frac{1}{2}} v\right\|_{L^{2}}^{2} \simeq\left\|\nabla^{b} v\right\|_{L^{2}}^{2}+\int_{\Omega} c|v|^{2} d x \simeq\|\nabla v\|_{L^{2}}^{2}+\int_{\Omega} c|v|^{2} d x .
$$

By Hardy's inequality we obtain the claim for $s=1$, provided $C_{-}$is sufficiently small, and by complex interpolation we conclude the proof (recalling the complex interpolation formula $\left[D\left(H^{\sigma_{0}}\right), D\left(H^{\sigma_{1}}\right)\right]_{\theta}=D\left(H^{\sigma_{\theta}}\right)$ with $\sigma_{\theta}=(1-\theta) \sigma_{0}+\theta \sigma_{1}$ which is valid for any selfadjoint operator $H$ ). 


\section{ViRIAL IDENTITY}

In [5] a virial identity for the Helmholtz equation with variable coefficients was obtained by adapting the Morawetz multiplier method. We show here how to modify the technique in order to prove the analogous virial identity for the nonlinear Schrödinger equation (1.1). To make sense of the formal manipulations, one needs some additional smoothness (e.g., $u \in H^{2}(\Omega)$ is enough), which can be obtained by an approximation procedure similar to the proof of the conservation of energy in Theorem 7.2 below; we omit the details. The identity is the following:

Proposition 3.1 (Virial Identity). Assume $a, b, c, f(z)$ are as in Theorem 1.1, let $u$ be a solution of (1.1) and $\psi: \mathbb{R}^{n} \rightarrow \mathbb{R}$ an arbitrary weight. Then the following identity holds:

$$
\begin{aligned}
\partial_{t}\left[\Im\left(a\left(\nabla \psi, \nabla^{b} u\right) u\right)\right]= & -\frac{1}{2} A^{2} \psi|u|^{2}+\Re\left(\alpha_{\ell m} \partial_{m}^{b} u \overline{\partial_{\ell}^{b} u}\right) \\
& -a(\nabla \psi, \nabla c)|u|^{2} \\
& +2 \Im\left(a_{j k} \partial_{k}^{b} u\left(\partial_{j} b_{\ell}-\partial_{\ell} b_{j}\right) a_{\ell m} \partial_{m} \psi \bar{u}\right) \\
& +A \psi[f(u) \bar{u}-2 F(u)] \\
& +\partial_{j}\left\{-\Re Q_{j}+2 F(u) a_{j k} \partial_{k} \psi+\Im\left[u_{t} \bar{u} a_{j k} \partial_{k} \psi\right]\right\},
\end{aligned}
$$

where

$$
\begin{gathered}
\alpha_{\ell m}=2 a_{j m} \partial_{j}\left(a_{\ell k} \partial_{k} \psi\right)-a_{j k} \partial_{k} \psi \partial_{j} a_{\ell m} \\
Q_{j}=a_{j k} \partial_{k}^{b} u \cdot\left[A^{b}, \psi\right] \bar{u}-\frac{1}{2} a_{j k}\left(\partial_{k} A \psi\right)|u|^{2}-a_{j k} \partial_{k} \psi\left[c|u|^{2}+a\left(\nabla^{b} u, \nabla^{b} u\right)\right] .
\end{gathered}
$$

Proof. We multiply both sides of (1.1) by the multiplier

$$
\left[A^{b}, \psi\right] \bar{u}=(A \psi) \bar{u}+2 a(\nabla \psi, \nabla u)
$$

and take real parts. We recall the following identity (which however can be checked directly with some lengthy but elementary computations) from Proposition 2.1 of $[5]$ :

$$
\begin{aligned}
\Re\left[\left(-A^{b} u+c u\right)\left[A^{b}, \psi\right] \bar{u}\right]= & -\frac{1}{2} A^{2} \psi|u|^{2}+\Re\left(\alpha_{\ell m} \partial_{m}^{b} u \overline{\partial_{\ell}^{b} u}\right) \\
& -a(\nabla \psi, \nabla c)|u|^{2} \\
& +2 \Im\left(a_{j k} \partial_{k}^{b} u\left(\partial_{j} b_{\ell}-\partial_{\ell} b_{j}\right) a_{\ell m} \partial_{m} \psi \bar{u}\right) \\
& -\Re \partial_{j} Q_{j},
\end{aligned}
$$

where $\alpha_{l m}$ are defined by (3.2) and $Q_{j}$ by (3.3). For the terms containing $f(u)$ we can write

$$
\Re\left(f(u)\left[A^{b}, \psi\right] \bar{u}\right)=A \psi[f(u) \bar{u}-2 F(u)]+\nabla \cdot\{2 F(u) a \nabla \psi\} .
$$

Indeed, by the assumptions on $f$, there exists a function $g:[0,+\infty) \rightarrow \mathbb{R}$ such that $f(z)=g\left(|z|^{2}\right) z$. As a consequence,

$$
\begin{aligned}
\nabla F(u) & =\nabla \int_{0}^{|u|} f(s) d s=\nabla \int_{0}^{|u|} g\left(s^{2}\right) s d s= \\
& =\frac{1}{2} \nabla \int_{0}^{|u|^{2}} g(t) d t=\Re\left(g\left(|u|^{2}\right) u \nabla \bar{u}\right)=\Re(f(u) \nabla \bar{u})= \\
& =\Re\left(f(u) \overline{\nabla^{b} u}\right),
\end{aligned}
$$


since $\Re(f(u) i b \bar{u})=0$. We conclude that

$$
\begin{aligned}
\Re\left[f(u)\left[A \psi \bar{u}+2 a\left(\nabla \psi, \nabla^{b} u\right)\right]\right] & =A \psi f(u) \bar{u}+2 \nabla \psi^{t} a \Re\left(f(u) \overline{\nabla^{b} u}\right)= \\
& =A \psi f(u) \bar{u}+2 \nabla \psi^{t} a \nabla F(u)= \\
& =A \psi f(u) \bar{u}+2[a \nabla \psi] \cdot \nabla F(u)= \\
& =A \psi[f(u) \bar{u}-2 F(u)]+\nabla \cdot\{2 F(u) a \nabla \psi\},
\end{aligned}
$$

and (3.5) is proved. Finally, for the terms containing $i u_{t}$ we have the identity

$$
\Re\left(i \partial_{t} u\left[A^{b}, \psi\right] \bar{u}\right)=\partial_{t}\left[-\Im a\left(\nabla \psi, \nabla^{b} u\right) u\right]+\nabla \cdot\left\{\Im\left(u_{t} \bar{u} a \nabla \psi\right)\right\} .
$$

This can be proved directly as follows:

$$
\begin{aligned}
\Re[ & \left.i u_{t}\left[A \psi \bar{u}+2 a\left(\nabla \psi, \nabla^{b} u\right)\right]\right]=-\Im\left[u_{t} \nabla \cdot(a \nabla \psi) \bar{u}+2 \nabla \psi^{t} a \overline{\nabla u} u_{t}-2 i \nabla \psi^{t} a b \bar{u} u_{t}\right]= \\
& =-\Im\left[-\nabla u_{t}^{t} a \nabla \psi \bar{u}-u_{t} \overline{\nabla u}^{t} a \nabla \psi+2 \nabla \psi^{t} a \overline{\nabla u} u_{t}-2 i \nabla \psi^{t} a b \bar{u} u_{t}+\nabla \cdot\left\{u_{t} \bar{u} a \nabla \psi\right\}\right]= \\
& =-\Im\left[\overline{\nabla u}_{t}^{t} a \nabla \psi u+\overline{\nabla u}^{t} a \nabla \psi u_{t}\right]+2 \Im\left[i \nabla \psi^{t} a(b \bar{u}) u_{t}\right]+\nabla \cdot\left\{\Im\left[u_{t} \bar{u} a \nabla \psi\right]\right\}= \\
& =-\Im\left[\partial_{t}\left(\overline{\nabla u}^{t} a \nabla \psi u\right)\right]+\Im\left[i \partial_{t}\left(\nabla \psi^{t} a \overline{b u} u\right)\right]+\nabla \cdot\left\{\Im\left[u_{t} \bar{u} a \nabla \psi\right]\right\}= \\
& =-\Im\left[\partial_{t}\left(\overline{\nabla u}^{t} a \nabla \psi u\right)\right]+\Im\left[i \partial_{t}\left(\nabla \psi^{t} a \overline{b u} u\right)\right]+\nabla \cdot\left\{\Im\left[u_{t} \bar{u} a \nabla \psi\right]\right\}= \\
& =\partial_{t}\left[-\Im a\left(\nabla \psi, \nabla^{b} u\right) u\right]+\nabla \cdot\left\{\Im\left[u_{t} \bar{u} a \nabla \psi\right]\right\} .
\end{aligned}
$$

Gathering (3.4), (3.5) and (3.6) we obtain (3.1).

\section{Proof of Theorems 1.1, 1.2: the Smoothing estimate}

Since the arguments for Theorems 1.1 and 1.2 largely overlap, we shall proceed with both proofs in parallel. The proof consists in integrating the virial identity (3.1) on $\Omega$ and estimating carefully all the terms. Note that some of the following computations are similar to those of Section 4 in [5].

Remark 4.1. At several steps, we shall need to assume that the constants $N / \nu-1$, $C_{a}, C_{I}, C_{c}, C_{b}, C_{-}$are small enough. We can give explicit conditions on the smallness required in Theorem 1.1 and in Theorem 1.2. In both the Theorems the smallness of $C_{-}$is only required in order to make $L$ a selfadjoint, nonpositive operator. In view of the magnetic Hardy inequality (2.11), it is sufficient to assume

$$
C_{-} \leq \frac{2 \sqrt{\nu}}{n-2}
$$

In Theorem 1.1 it is sufficient that

$$
\frac{N}{\nu} \leq \sqrt{\frac{n^{2}+2 n+15}{6(n+2)}} \quad \text { for } 3 \leq n \leq 25, \quad \frac{N}{\nu}<\frac{7 n-1}{3(n+3)} \quad \text { for } n \geq 26
$$

and that the following quantities are positive:

$$
\begin{gathered}
\frac{K_{0}}{2} \nu^{2}-\frac{5 N^{2} C_{b}+12 n C_{a}\left(N+C_{a}\right)+C_{c}}{\delta}>0, \\
\frac{n-1}{3 n} \nu^{2}-\frac{5 N^{2} C_{b}+24 N C_{a}}{\delta}>0, \quad\left(n-\frac{N}{\nu}\right)-\frac{n}{n-1} \nu C_{a}>0,
\end{gathered}
$$

where

$$
K_{0}:=\frac{n-1}{6}-\frac{n+3}{2} \frac{N}{\nu}+n>0 .
$$

We remark that $n-N / \nu>0$ thanks to (4.2). On the other hand, the condition $K_{0}>0$ is equivalent to the second equation in (4.2) and is implied by the first equation in (4.2) in the case $n \leq 26$. 
In Theorem 1.2 it is sufficient that the following quantities are positive:

$$
\begin{gathered}
\frac{\left(1-C_{I}\right)^{2}}{78}-8 \delta^{-1}\left[C_{c}+9 C_{I}+41 C_{a}\left(N+C_{a}\right)\right]-9 \delta^{-1} N^{2} C_{b}>0 \\
\frac{\left(1-C_{I}\right)^{2}}{6}-13 \delta^{-1}\left[C_{c}+38 C_{a}\left(N+C_{a}\right)\right]-9 \delta^{-1} N^{2} C_{b}>0 \\
\left(n-\frac{N}{\nu}\right)-\frac{n}{n-1} \nu C_{a}>0 .
\end{gathered}
$$

The proof is divided into several steps.

\subsection{Choice of the weight $\psi$. Define}

$$
\psi_{1}(r)=\int_{0}^{r} \psi_{1}^{\prime}(s) d s
$$

where

$$
\psi_{1}^{\prime}(r)= \begin{cases}\frac{n-1}{2 n} r, & r \leq 1 \\ \frac{1}{2}-\frac{1}{2 n r^{n-1}}, & r>1 .\end{cases}
$$

Then $\psi$ is the radial function, depending on a scaling parameter $R>0$,

$$
\psi(|x|) \equiv \psi_{R}(|x|):=R \psi_{1}\left(\frac{|x|}{R}\right) .
$$

Here and in the following, with a slight abuse, we shall use the same letter $\psi$ to denote a function $\psi(r)$ defined for $r \in \mathbb{R}^{+}$and the radial function $\psi(x)=\psi(|x|)$ defined on $\mathbb{R}^{n}$. We compute the first radial derivatives $\psi^{(j)}(r)=\left(\frac{x}{|x|} \cdot \nabla\right)^{j} \psi(x)$ for $|x|>0$ :

$$
\psi^{\prime}(x)= \begin{cases}\frac{n-1}{2 n} \cdot \frac{|x|}{R}, & |x| \leq R \\ \frac{1}{2}-\frac{R^{n-1}}{2 n|x|^{n-1}}, & |x|>R\end{cases}
$$

which can be equivalently written as

$$
\psi^{\prime}(x)=\frac{|x|}{2 n R}\left[n \frac{R}{R \vee|x|}-\left(\frac{R}{R \vee|x|}\right)^{n}\right]
$$

and implies in particular

$$
0 \leq \psi^{\prime} \leq \frac{1}{2}
$$

Then we have

$$
\begin{gathered}
\psi^{\prime \prime}(x)=\frac{n-1}{2 n} \cdot \frac{R^{n-1}}{(R \vee|x|)^{n}}=\frac{n-1}{2 n} \cdot \begin{cases}\frac{1}{R} & |x| \leq R \\
\frac{R^{n-1}}{|x|^{n}} & |x|>R,\end{cases} \\
\psi^{\prime \prime \prime}(x)=-\frac{n-1}{2} \frac{R^{n-1}}{|x|^{n+1}} \mathbf{1}_{|x| \geq R} \\
\psi^{I V}(x)=\frac{n^{2}-1}{2} \cdot \frac{R^{n-1}}{|x|^{n+2}} \mathbf{1}_{|x| \geq R}-\frac{n-1}{2} \frac{1}{R^{2}} \delta_{|x|=R} . \\
\psi^{\prime \prime}-\frac{\psi^{\prime}}{|x|}= \begin{cases}0 & |x| \leq R \\
-\frac{1}{2|x|}\left(1-\frac{R^{n-1}}{|x|^{n-1}}\right) & |x|>R .\end{cases}
\end{gathered}
$$

Moreover the function (see (2.2))

$$
A \psi=\widehat{a} \psi^{\prime \prime}+\frac{\bar{a}-\widehat{a}}{|x|} \psi^{\prime}+a_{\ell m ; \ell} \widehat{x}_{m} \psi^{\prime} .
$$

is continuous and piecewise Lipschitz. 
4.2. Estimate of the terms in $|u|^{2}$. In this section we consider the terms

$$
I_{|u|^{2}}=-\frac{1}{2} A^{2} \psi|u|^{2}-a(\nabla \psi, \nabla c)|u|^{2} .
$$

We compute the quantity $A^{2} \psi$ : after some long but elementary computations (see [5]) we have

$$
A^{2} \psi(x)=S(x)+R(x)
$$

where

$$
\begin{aligned}
S(x)= & \widehat{a}^{2} \psi^{I V}+\left[2 \bar{a} \widehat{a}-6 \widehat{a}^{2}+4|a \widehat{x}|^{2}\right] \frac{\psi^{\prime \prime \prime}}{|x|}+ \\
& +\left[2 a_{\ell m} a_{\ell m}+\bar{a}^{2}-6 \bar{a} \widehat{a}+15 \widehat{a}^{2}-12|a \widehat{x}|^{2}\right]\left(\frac{\psi^{\prime \prime}}{|x|^{2}}-\frac{\psi^{\prime}}{|x|^{3}}\right)
\end{aligned}
$$

and

$$
\begin{aligned}
R(x)= & \widehat{a} a_{\ell m ; \ell} \widehat{x}_{m} \psi^{\prime \prime \prime}+(\bar{a}-\widehat{a}) a_{j k ; j} \widehat{x}_{k}\left(\frac{\psi^{\prime \prime}}{|x|}-\frac{\psi^{\prime}}{|x|^{2}}\right)+ \\
& +\left[\partial_{j}\left(a_{j k} a_{\ell m ; k} \widehat{x}_{\ell} \widehat{x}_{m}\right)+\partial_{j}\left(a_{j k} a_{\ell m}\right) \partial_{k}\left(\widehat{x}_{\ell} \widehat{x}_{m}\right)\right]\left(\psi^{\prime \prime}-\frac{\psi^{\prime}}{|x|}\right)+(A \bar{a}) \frac{\psi^{\prime}}{|x|}+ \\
& +2 a_{j k} a_{\ell m ; k} \widehat{x}_{\ell} \widehat{x}_{m} \widehat{x}_{j}\left(\psi^{\prime \prime \prime}-\frac{\psi^{\prime \prime}}{|x|}\right)+2 a\left(\nabla \bar{a}, \nabla \frac{\psi^{\prime}}{|x|}\right)+ \\
& +A\left(a_{\ell m ; \ell} \widehat{x}_{m} \psi^{\prime}\right) .
\end{aligned}
$$

The remainder $R(x)$ can be estimated as follows: recalling that, by definition of $\psi$, we have

$$
\left|\psi^{\prime}\right| \leq \frac{|x|}{2(R \vee|x|)}, \quad\left|\psi^{\prime \prime}\right| \leq \frac{n-1}{2 n(R \vee|x|)}, \quad\left|\psi^{\prime \prime \prime}\right| \leq \frac{n-1}{2|x|(R \vee|x|)}
$$

and using assumption (1.6), we find that

$$
|R(x)| \leq \frac{12 n C_{a}\left(N+C_{a}\right)}{|x|\langle x\rangle^{1+\delta}(R \vee|x|)}
$$

4.2.1. Proof of Theorem 1.1. We prove that, assuming (1.9), (1.6), (1.3), (4.2), we have

$$
\begin{aligned}
\int_{\Omega} \int_{0}^{T} I_{|u|^{2}} d t d x \geq & \frac{n-1}{2} \nu \frac{1}{R^{2}} \int_{\Omega_{=R}} \widehat{a}\|u\|_{L_{T}^{2}} d S \\
& -\left[\frac{n+3}{2} N-n \nu\right](n-1) \int_{\Omega_{\geq R}} \widehat{a} \frac{R^{n-1}}{|x|^{n+2}}\|u\|_{L_{T}^{2}} d x \\
& -\left(12 n C_{a}\left(N+C_{a}\right)+C_{c}\right) \delta^{-1}\|u\|_{\dot{X}_{x} L_{T}^{2}}^{2}
\end{aligned}
$$

We focus on the main term $S(x)$. With our choice of the weight $\psi$ we have in the region $|x| \leq R$

while in the region $|x|>R$

$$
S(x)=-\frac{n-1}{2} \widehat{a}^{2} \frac{1}{R^{2}} \delta_{|x|=R}
$$

$$
\begin{aligned}
S(x)= & (n-1)\left[\frac{n+3}{2} \widehat{a}-\bar{a}\right] \widehat{a} \frac{R^{n-1}}{|x|^{n+2}}-2(n-1)\left[|a \widehat{x}|^{2}-\widehat{a}^{2}\right] \frac{R^{n-1}}{|x|^{n+2}} \\
& -\left[2 a_{\ell m} a_{\ell m}+\bar{a}^{2}-6 \bar{a} \widehat{a}+15 \widehat{a}^{2}-12|a \widehat{x}|^{2}\right]\left(1-\left(\frac{R}{|x|^{n-1}}\right) \frac{1}{2|x|^{3}} .\right.
\end{aligned}
$$

Note that $a_{\ell m} a_{\ell m}$ is the square of the Hilbert-Schmidt norm of the matrix $a(x)$. We deduce from assumption (1.3)

$$
n N \geq \bar{a} \geq n \nu, \quad N \geq|a \widehat{x}| \geq \widehat{a} \geq \nu, \quad a_{\ell m} a_{\ell m} \geq n \nu^{2},
$$

so that

$$
S(x) \leq-\frac{n-1}{2} \nu \widehat{a} \frac{1}{R^{2}} \delta_{|x|=R} \quad \text { for }|x| \leq R .
$$

On the other hand, we have

$2|a(x)|_{H S}^{2}+\bar{a}^{2}-6 \bar{a}(x) \widehat{a}(x)+15 \widehat{a}^{2}(x)-12|a(x) \widehat{x}|^{2} \geq\left(2 n+n^{2}+15\right) \nu^{2}-6(n+2) N^{2} \geq 0$ 
by (4.2) (note that the second condition in (4.2) implies the first one when $n \geq 26$ ), thus we get

$$
S(x) \leq(n-1)\left[\frac{n+3}{2} N-n \nu\right] \widehat{a} \frac{R^{n-1}}{|x|^{n+2}} \quad \text { for }|x| \geq R .
$$

Now we can estimate from below the integral

$$
-\int_{\Omega} \int_{0}^{T} A^{2} \psi|u|^{2} d t d x=-\int_{\Omega} A^{2} \psi\|u(x)\|_{L_{T}^{2}}^{2} d x=I+I I
$$

where

$$
I=-\int_{\Omega} S(x)\|u(x)\|_{L_{T}^{2}}^{2} d x, \quad I I=-\int_{\Omega} R(x)\|u(x)\|_{L_{T}^{2}}^{2} d x .
$$

By (4.14) and (2.7) we have immediately for any $R>0$

$$
I I \geq-24 n \delta^{-1} C_{a}\left(N+C_{a}\right)\|u\|_{\dot{X}_{x} L_{T}^{2}}^{2} .
$$

Note that we must first integrate in time over $[0, T]$, then in space over $\Omega_{=R}$ and finally divide by $R^{2}$ and take the sup in $R>0$; this gives a reverse norm $\dot{X}_{x} L_{t}^{2}$ in the previous estimate. Concerning the $S(x)$ term $I$, we have by (4.18), (4.20)

$$
I \geq \frac{n-1}{2} \nu \frac{1}{R^{2}} \int_{\Omega_{=R}} \widehat{a}\|u\|_{L_{T}^{2}}^{2} d S-\left[\frac{n+3}{2} N-n \nu\right](n-1) \int_{\Omega_{\geq R}} \widehat{a} \frac{R^{n-1}}{|x|^{n+2}}\|u\|_{L_{T}^{2}}^{2} d x
$$

for all $R>0$.

It remains to consider the second term in (4.11); we have

$$
-a(\nabla \psi, \nabla c)|u|^{2}=-a(\widehat{x}, \nabla c) \psi^{\prime}|u|^{2} \geq-\frac{C_{c}}{|x|^{2}\langle x\rangle^{1+\delta}} \psi^{\prime}|u|^{2}
$$

thanks to assumption (1.9). Since $0<\psi^{\prime}<1 / 2$, by estimate (2.7) we obtain

$$
-\int_{\Omega} \int_{0}^{T} a(\nabla \psi, \nabla c)|u|^{2} d t d x \geq-C_{c} \delta^{-1}\|u\|_{\dot{X}_{x} L_{T}^{2}}^{2}
$$

Collecting (4.22), (4.21), and (4.24) we get (4.15).

4.2.2. Proof of Theorem 1.2. We prove that, assuming (1.3), (1.6), (1.16), (1.9), we have for all $R>1$

$$
\begin{aligned}
\int_{\Omega} \int_{0}^{T} I_{|v|^{2}} d t d x \geq & \left(1-C_{I}\right) \frac{1}{R^{2}} \int_{\Omega_{=R}}\|u\|_{L_{T}^{2}}^{2} d S \\
& -8 \delta^{-1}\left[C_{c}+9 C_{I}+41 C_{a}\left(N+C_{a}\right)\right]\|u\|_{X L_{T}^{2}}^{2} \\
& -13 \delta^{-1}\left[C_{c}+36 C_{a}\left(N+C_{a}\right)\right]\left\|\nabla^{b} u\right\|_{Y L_{T}^{2}}^{2} .
\end{aligned}
$$

Writing $a(x)=I+q(x)$ i.e. $q_{\ell m}:=a_{\ell m}-\delta_{\ell m}$ we have, with the notations $\widehat{q}=$ $q_{\ell m} \widehat{x}_{\ell} \widehat{x}_{m}$ and $\bar{q}=q_{\ell \ell}$,

$$
a_{\ell m} a_{\ell m}=\delta_{\ell m} \delta_{\ell m}+2 \delta_{\ell m} q_{\ell m}+q_{\ell m} q_{\ell m}=3+2 \bar{q}+q_{\ell m} q_{\ell m}
$$

and also

$$
\widehat{a}=1+\widehat{q}, \quad \bar{a}=3+\bar{q}, \quad|a \widehat{x}|^{2}=1+2 \widehat{q}+|q \widehat{x}|^{2} .
$$

Note that $|q|=|a(x)-I| \leq C_{I}\langle x\rangle^{-\delta}<1$ by assumption (1.16), which implies

$$
|\bar{q}| \leq 3 C_{I}\langle x\rangle^{-\delta}, \quad|\widehat{q}| \leq C_{I}\langle x\rangle^{-\delta}, \quad|q \widehat{x}| \leq C_{I}\langle x\rangle^{-\delta}
$$

so that

$$
\begin{aligned}
2 a_{\ell m} a_{\ell m}+\bar{a}^{2}-6 \bar{a} \widehat{a}+15 \widehat{a}^{2}-12|a \widehat{x}|^{2} & =4 \bar{q}-12 \widehat{q}+2 q_{\ell m} q_{\ell m}+\bar{q}^{2}-6 \bar{q} \widehat{q}+15 \widehat{q}^{2}-12|q \widehat{x}|^{2} \\
& \geq 4 \bar{q}-12 \widehat{q}-6 \bar{q} \widehat{q}-12|q \widehat{x}|^{2} \geq-46 C_{I}\langle x\rangle^{-\delta} .
\end{aligned}
$$

We have also $1-C_{I} \leq \widehat{a} \leq 1+C_{I}$ so that $(n=3)$

$$
-\frac{n-1}{2} \widehat{a}^{2} \leq-\left(1-C_{I}\right)^{2}, \quad\left(\frac{n+3}{2} \widehat{a}-\bar{a}\right) \widehat{a} \leq 6 C_{I}\left(1+C_{I}\right)<12 C_{I}
$$

Thus under the assumptions of Theorem 1.2 we obtain the estimates

$$
S(x) \leq-\left(1-C_{I}\right)^{2} \frac{1}{R^{2}} \delta_{|x|=R} \quad \text { for }|x| \leq R
$$


and

$$
S(x) \leq 24 C_{I}\left[\frac{R^{2}}{|x|^{5}}+\frac{1}{|x|^{3}\langle x\rangle^{\delta}}\right] \quad \text { for }|x|>R .
$$

Now we can estimate from below the integral

$$
-\int_{\Omega} \int_{0}^{T} A^{2} \psi|u|^{2} d t d x=-\int_{\Omega} A^{2} \psi\|u(x)\|_{L_{T}^{2}}^{2} d x=I+I I
$$

where

$$
I=-\int_{\Omega} S(x)\|u(x)\|_{L_{T}^{2}}^{2} d x, \quad I I=-\int_{\Omega} R(x)\|u(x)\|_{L_{T}^{2}}^{2} d x .
$$

Concerning the $S(x)$ term $I$, using (2.6) and (2.8) in (4.26), (4.27), we have for all $R>1$

$$
I \geq\left(1-C_{I}\right)^{2} \frac{1}{R^{2}} \int_{\Omega_{=R}}\|u\|_{L_{T}^{2}}^{2} d S-72 C_{I} \delta^{-1}\|u\|_{X_{x} L_{T}^{2}}^{2} .
$$

We estimate the now the $I I$-term: for all $R>1$, thanks to (4.14), we have

$$
\begin{aligned}
I I & \geq-36 C_{a}\left(N+C_{a}\right) \int_{0}^{T} \int_{\Omega}|x|^{-1}\langle x\rangle^{-1-\delta}(R \vee|x|)^{-1}|u(t, x)|^{2} d x d t \\
& \geq-36 C_{a}\left(N+C_{a}\right) \int_{0}^{T}\left[\int_{\Omega_{\leq 1}}+\int_{\Omega_{\geq 1}}\right]|x|^{-2}\langle x\rangle^{-1-\delta}|u(t, x)|^{2} d x d t
\end{aligned}
$$

We observe that, thanks to (2.8), we have

$$
\int_{0}^{T} \int_{\Omega_{\geq 1}} \frac{|u|^{2}}{|x|^{2}\langle x\rangle^{1+\delta}} d x d t=\int_{\Omega_{\geq 1}} \frac{\|u(x)\|_{L_{T}^{2}}}{|x|^{2}\langle x\rangle^{1+\delta}} \leq 2 \delta^{-1}\|u\|_{X L_{T}^{2}} .
$$

Moreover, thanks to (2.11) and (2.4), we estimate

$$
\begin{aligned}
\int_{0}^{T} \int_{\Omega_{\leq 1}} \frac{|u|^{2}}{|x|^{2}\langle x\rangle^{1+\delta}} d x d t & \leq \int_{0}^{T} \int_{\Omega_{\leq 1}}|x|^{-2}|u|^{2} d x d t \leq 4 \int_{0}^{T} \int_{\Omega_{\leq 1}}\left|\nabla^{b} u\right|^{2} d x d t \\
& =4\left\|\nabla^{b} u\right\|_{L^{2}\left(\Omega_{\leq 1}\right) L_{T}^{2}}^{2}
\end{aligned}
$$

Gathering (4.30) and (4.31), we have

$$
\int_{0}^{T} \int_{\Omega} \frac{|u|^{2}}{|x|^{2}\langle x\rangle^{1+\delta}} d x d t \leq 2 \delta^{-1}\|u\|_{X L_{T}^{2}}^{2}+4\left\|\nabla^{b} u\right\|_{L^{2}\left(\Omega_{\leq 1}\right) L_{T}^{2}}^{2} .
$$

We get immediately from (4.29) and (4.32) that

$$
I I \geq-324 \delta^{-1} C_{a}\left(N+C_{a}\right)\left[\|u\|_{X L_{T}^{2}}^{2}+\left\|\nabla^{b} u\right\|_{L^{2}\left(\Omega_{\leq 1}\right) L_{T}^{2}}^{2}\right] .
$$

We consider the second term in (4.11); thanks to (1.9) and (4.32) we have

$$
\begin{aligned}
-\int_{0}^{T} \int_{\Omega} a(\nabla \psi, \nabla c)|u|^{2} d x d t & \geq-\int_{0}^{T} \int_{\Omega} \frac{C_{c}}{2} \frac{|u|^{2}}{|x|^{2}\langle x\rangle^{1+\delta}} d x d t \\
& \geq-C_{c} \delta^{-1}\|u\|_{X L_{T}^{2}}^{2}-2 C_{c}\left\|\nabla^{b} u\right\|_{L^{2}\left(\Omega_{\leq 1}\right) L_{T}^{2}}^{2} .
\end{aligned}
$$

Recalling (4.33), (4.28) and (4.34) we finally get

$$
\begin{aligned}
\int_{\Omega} \int_{0}^{T} I_{|u|^{2}} d t d x \geq & \left(1-C_{I}\right)^{2} \frac{1}{R^{2}} \int_{\Omega=R}\|u\|_{L_{T}^{2}}^{2} d S \\
& -\left(72 C_{I} \delta^{-1}+\delta^{-1} C_{c}\right)\|u\|_{X L_{T}^{2}}^{2}-2 C_{c}\left\|\nabla^{b} u\right\|_{L^{2}\left(\Omega_{\leq 1}\right) L_{T}^{2}}^{2} \\
& -324 \delta^{-1} C_{a}\left(N+C_{a}\right)\left[\|u\|_{X L_{T}^{2}}^{2}+\left\|\nabla^{b} u\right\|_{L^{2}\left(\Omega_{\leq 1}\right) L_{T}^{2}}^{2}\right]
\end{aligned}
$$

whence, noticing that $\|w\|_{L^{2}\left(\Omega_{\leq 1}\right)} \leq \sqrt{2}\|w\|_{Y}$, we have (4.25) for all $R>1$. 
4.3. Estimate of the terms in $\left|\nabla^{b} u\right|^{2}$. For such terms, using assumption (1.6), we shall prove for all $R>0$ the estimate

$$
\int_{\Omega} \alpha_{l m} \Re\left(\partial_{l}^{b} u \overline{\partial_{m}^{b} u}\right) d x \geq \frac{n-1}{n R} \nu^{2} \int_{\Omega_{\leq R}}\left\|\nabla^{b} u(x)\right\|_{L_{T}^{2}}^{2} d x-24 N C_{a} \delta^{-1}\left\|\nabla^{b} u\right\|_{Y_{x} L_{T}^{2}}^{2},
$$

where $\alpha_{l m}$ are the quantities defined in (3.2). The computations here are very similar to those in Section 4 of [5]. We split the quantities $\alpha_{\ell m}$ as

$$
\alpha_{\ell m}(x)=s_{\ell m}(x)+r_{\ell m}(x)
$$

where the remainder $r_{\ell m}$ gathers all terms containing some derivative of the $a_{j k}$. Since the weight $\psi$ is radial, we have

$$
s_{\ell m}(x)=2 a_{j m} a_{\ell k} \widehat{x}_{j} \widehat{x}_{k}\left(\psi^{\prime \prime}-\frac{\psi^{\prime}}{|x|}\right)+2 a_{j m} a_{j \ell} \frac{\psi^{\prime}}{|x|}
$$

while

$$
r_{\ell m}(x)=\left[2 a_{j m} a_{\ell k ; j}-a_{j k} a_{\ell m ; j}\right] \widehat{x}_{k} \psi^{\prime}
$$

We estimate directly

$$
\left|r_{\ell m}(x) \Re\left(\partial_{\ell}^{b} u \overline{\partial_{m}^{b} u}\right)\right| \leq 3|a(x)|\left|a^{\prime}(x)\right|\left|\nabla^{b} u(x)\right|^{2}
$$

and by assumption (1.6) we obtain

$$
\left|r_{\ell m}(x) \Re\left(\partial_{\ell}^{b} u \overline{\partial_{m}^{b} u}\right)\right| \leq 3 N C_{a}\langle x\rangle^{-1-\delta}\left|\nabla^{b} u\right|^{2} .
$$

Integrating in $t \in[0, T]$ first and then in $x \in \Omega$, we get

$$
\begin{aligned}
\int_{\Omega} \int_{0}^{T}\left|r_{\ell m}(x) \Re\left(\partial_{\ell}^{b} u \overline{\partial_{m}^{b} u}\right)\right| d t d x & \leq 3 N C_{a} \int_{\Omega}\langle x\rangle^{-1-\delta} \int_{0}^{T}\left|\nabla^{b} u\right|^{2} d t d x \\
& =3 N C_{a} \int_{\Omega}\langle x\rangle^{-1-\delta}\left\|\nabla^{b} u(x)\right\|_{L_{T}^{2}}^{2} d x .
\end{aligned}
$$

Thus, using (2.9), we obtain the estimate

$$
\int_{\Omega} \int_{0}^{T}\left|r_{\ell m} \Re\left(\partial_{\ell}^{b} u \overline{\partial_{m}^{b} u}\right)\right| d t d x \leq 24 N C_{a} \delta^{-1}\left\|\nabla^{b} u\right\|_{Y_{x} L_{T}^{2}}^{2} .
$$

Concerning the terms $s_{\ell m}$, in the region $|x|>R$ we have

$$
s_{\ell m}(x)=\left[a_{j m} a_{j \ell}-a_{j m} a_{\ell k} \widehat{x}_{j} \widehat{x}_{k}\right] \frac{1}{|x|}+\frac{R^{n-1}}{|x|^{n}} a_{j m} a_{\ell k} \widehat{x}_{j} \widehat{x}_{k}-a_{j m} a_{j \ell} \frac{R^{n-1}}{n|x|^{n}}
$$

so that, in the sense of positivity of matrices,

$$
s_{\ell m}(x) \geq\left[a_{j m} a_{j \ell}-a_{j m} a_{\ell k} \widehat{x}_{j} \widehat{x}_{k}\right] \frac{n-1}{n|x|} \geq 0 \quad \text { for }|x|>R
$$

(indeed, one has $a_{j m} a_{j \ell} \geq a_{j m} a_{\ell k} \widehat{x}_{j} \widehat{x}_{k}$ as matrices); on the other hand, in the region $|x| \leq R$ we have

$$
s_{\ell m}(x)=a_{j m} a_{j \ell} \frac{n-1}{n R} \quad \text { for }|x| \leq R .
$$

Thus, by the assumption $a(x) \geq \nu I$, one has for all $x$

$$
s_{\ell m}(x) \Re\left(\partial_{\ell}^{b} u \overline{\partial_{m}^{b} u}\right) \geq \frac{n-1}{n R} \nu^{2} \mathbf{1}_{|x| \leq R}(x)\left|\nabla^{b} u\right|^{2} .
$$

Integrating (4.37) with respect to $t \in[0, T]$ and $x \in \Omega$, and recalling (4.36), we conclude the proof of (4.35).

4.4. Estimate of the magnetic terms. We now consider the terms

$$
I_{b}:=2 \Im\left[a_{j k} \partial_{k}^{b} u\left(\partial_{j} b_{\ell}-\partial_{\ell} b_{j}\right) a_{\ell m} \partial_{m} \psi \bar{u}\right]=2 \Im\left[(d b \cdot a \widehat{x}) \cdot\left(a \nabla^{b} u\right) \bar{u} \psi^{\prime}\right]
$$

where the identity holds for any radial $\psi$, while $d b$ is the matrix

$$
d b=\left[\partial_{j} b_{\ell}-\partial_{\ell} b_{j}\right]_{j, \ell=1}^{n} .
$$


4.4.1. Proof of Theorem 1.1. We shall prove the estimate

$$
\int_{\Omega} \int_{0}^{T}\left|I_{b}\right| d x \leq 5 \delta^{-1} N^{2} C_{b}\left(\left\|\nabla^{b} u\right\|_{\dot{Y}_{x} L_{T}^{2}}^{2}+\|u\|_{\dot{X}_{x} L_{T}^{2}}^{2}\right) .
$$

Indeed, since $0 \leq \psi^{\prime} \leq 1 / 2$ and $|a(x)| \leq N$, by (1.7) we have

$$
\left|I_{b}(x)\right| \leq 2 N^{2}|d b(x)| \cdot\left|\nabla^{b} u\right||u| \psi^{\prime} \leq N^{2} \frac{\left|\nabla^{b} u\right||u|}{|x|^{2+\delta}+|x|^{2-\delta}} .
$$

We integrate in $t \in[0, T]$, then in $x \in \Omega$, and we use the Hölder inequality in time:

$$
\int_{\Omega} \int_{0}^{T}\left|I_{b}(x)\right| d t d x \leq N^{2} \int_{\Omega} \int_{0}^{T} \frac{\left|\nabla^{b} u\right||u|}{|x|^{2+\delta}+|x|^{2-\delta}} d t d x \leq N^{2} \int_{\Omega} \frac{\left\|\nabla^{b} u\right\|_{L_{T}^{2}}\|u\|_{L_{T}^{2}}}{|x|^{2+\delta}+|x|^{2-\delta}} d x
$$

and by estimate (2.10) we obtain (4.38).

4.4.2. Proof of Theorem 1.2. In this case we prove the estimate

$$
\int_{\Omega} \int_{0}^{T}\left|I_{b}\right| d t d x \leq 9 \delta^{-1} N^{2} C_{b}\left(\left\|\nabla^{b} u\right\|_{Y_{x} L_{T}^{2}}^{2}+\|u\|_{X_{x} L_{T}^{2}}^{2}\right) .
$$

The proof is completely analogous to the previous one, using (1.17) and (2.13).

4.5. Estimate of the terms containing $f(u)$. We prove here that there exists a $\gamma_{0}>0$ such that

$$
A \psi[f(u) \bar{u}-2 F(u)] \geq \frac{\gamma_{0}}{R \vee|x|}[f(u) \bar{u}-2 F(u)]
$$

Thanks to (1.13), it is sufficient to check the pointwise inequality

$$
A \psi(x) \geq \frac{\gamma_{0}}{R \vee|x|} .
$$

Indeed, for $|x| \leq R$,

$$
\widehat{a} \psi^{\prime \prime}+\frac{\bar{a}-\widehat{a}}{|x|} \psi^{\prime}=\frac{n-1}{2 n}\left[\frac{\widehat{a}}{R}+\frac{\bar{a}-\widehat{a}}{R}\right]=\frac{n-1}{2 n} \frac{\bar{a}}{R}
$$

while for $|x|>R$

$$
\widehat{a} \psi^{\prime \prime}+\frac{\bar{a}-\widehat{a}}{|x|} \psi^{\prime}=\frac{\widehat{a}}{|x|} \frac{n-1}{2 n} \frac{R^{n-1}}{|x|^{n-1}}+\frac{\bar{a}-\widehat{a}}{|x|}\left(\frac{1}{2}-\frac{1}{2 n} \frac{R^{n-1}}{|x|^{n-1}}\right) \geq \frac{\bar{a}-\widehat{a}}{|x|} \frac{n-1}{2 n} .
$$

Moreover, by (1.6),

$$
a_{l m ; l} \widehat{x}_{m} \psi^{\prime} \geq-\frac{C_{a}}{\langle x\rangle^{1+\delta}}\left|\psi^{\prime}\right| \geq-\frac{C_{a}}{|x|}\left|\psi^{\prime}\right|
$$

Summing up we get

$$
\begin{aligned}
A \psi & \geq \begin{cases}\frac{n-1}{2 n R}\left(\bar{a}-C_{a}\right) & |x| \leq R \\
\frac{1}{2|x|}\left[\frac{n-1}{n}(\bar{a}-\widehat{a})-C_{a}\right] & |x|>R\end{cases} \\
& \geq \frac{\gamma_{0}}{R \vee|x|},
\end{aligned}
$$

for any $\gamma_{0}>0$ such that

$$
\gamma_{0}< \begin{cases}\frac{n-1}{2 n}\left(\bar{a}-C_{a}\right) & |x| \leq R \\ \frac{1}{2}\left[\frac{n-1}{n}(\bar{a}-\widehat{a})-C_{a}\right] & |x|>R\end{cases}
$$

which is possible provided $C_{a}$ is so small that $C_{a}<\frac{n-1}{n}(\bar{a}(x)-\widehat{a}(x))$ (see Remark 4.1). 
4.6. Estimate of the boundary terms. We now prove that

$$
\int_{\Omega} \partial_{j}\left\{-\Re Q_{j}+2 F(u) a_{j k} \partial_{k} \psi+\Im\left[u_{t} \bar{u} a_{j k} \partial_{k} \psi\right]\right\} d x \geq 0 .
$$

Indeed, proceeding exactly as in [5], we see that assumption (1.14) implies

$$
\int_{\Omega} \partial_{j} \Re Q_{j} d x \leq 0 .
$$

Moreover, at any fixed $t \in[0, T]$ we have

$$
\int_{\Omega} \nabla \cdot\left\{2 F(u) a \nabla \psi+\Im\left[u_{t} \bar{u} a \nabla \psi\right]\right\}=0 .
$$

To see this, we integrate $\nabla \cdot\left\{2 F(u) a \nabla \psi+\Im\left[u_{t} \bar{u} a \nabla \psi\right]\right\}$ over the set $\Omega \cap\{|x| \leq R\}$ and let $R \rightarrow+\infty$ : the integral over $|x|=R$ tends to 0 since $a \nabla \psi \in L^{\infty}(\Omega)$ and thanks to (1.10)

$$
|F(u)| \leq\left|\int_{0}^{|u|} f(s) d s\right| \lesssim|u|^{\gamma+1} \in L^{1}(\Omega)
$$

(recall that $u \in H_{0}^{1}(\Omega)$ ), while the integral over $\partial \Omega$ vanishes by the Diriclet boundary condition since $F(0)=0$.

4.7. Estimate of the derivative term. We finally estimate the term at the left hand side of (3.1). We need the following Lemma:

Lemma 4.1. Let $v \in H_{0}^{1}(\Omega)$ and $\psi: \mathbb{R}^{n} \rightarrow \mathbb{R}$ be such that $\nabla \psi$ and $|x| A \psi$ are bounded. Then there exist $C=C\left(\|a\|_{L^{\infty}},\|\nabla \psi\|_{L^{\infty}},\||x| A \psi\|_{L^{\infty}}\right)>0$ such that

$$
\left|\int_{\Omega} a\left(\nabla \psi, \nabla^{b} v\right) v d x\right| \leq C\|v\|_{\dot{H}^{\frac{1}{2}}}^{2},
$$

Proof. Define for $f, g \in C_{c}^{\infty}(\Omega)$

$$
T(f, g):=\int_{\Omega} \nabla \psi(x) \cdot a(x) \overline{\nabla^{b} f(x)} g(x) d x=\int_{\Omega}[a(x) \nabla \psi(x)] \cdot \overline{\nabla^{b} f(x)} g(x) d x .
$$

We have trivially

$$
|T(f, g)| \leq \int_{\Omega}\left|[a(x) \nabla \psi(x)] \cdot \overline{\nabla^{b} f(x)} g(x)\right| d x \leq C\left\|\nabla^{b} f\right\|_{L^{2}(\Omega)}\|g\|_{L^{2}(\Omega)}
$$

with $C=\|a \nabla \psi\|_{L^{\infty}}$. On the other hand, integration by parts gives

$$
\begin{aligned}
|T(f, g)|=\left|\int_{\mathbb{R}^{n}}[a(x) \nabla \psi(x)] \overline{\nabla^{b} f(x)} g(x) d x\right|= \\
=\mid \int_{\mathbb{R}^{n}}[a(x) \nabla \psi(x)] \nabla^{b} g(x) \overline{f(x)} d x+\int_{\mathbb{R}^{n}} \nabla \cdot[a(x) \nabla \psi(x)] g(x) \overline{f(x)} d x+ \\
\quad-\int_{\mathbb{R}^{n}} \nabla \cdot\{[a(x) \nabla \psi(x)] g(x) \overline{f(x)}\} d x \mid .
\end{aligned}
$$

Discarding the divergence term and using the boundedness of $|x| A \psi$ we have, for some $C=C\left(\|a\|_{L^{\infty}},\|\nabla \psi\|_{L^{\infty}},\||x| A \psi\|_{L^{\infty}}\right)>0$,

$$
|T(f, g)| \leq C\left[\|f\|_{L^{2}(\Omega)}\left\|\nabla^{b} g\right\|_{L^{2}(\Omega)}+\|f\|_{L^{2}(\Omega)}\left\||x|^{-1} g\right\|_{L^{2}(\Omega)}\right]
$$

which implies, using the magnetic Hardy inequality (2.11),

$$
|T(f, g)| \leq C\|f\|_{L^{2}(\Omega)}\left\|\nabla^{b} g\right\|_{L^{2}(\Omega)}
$$

for a different $C=C\left(\|a\|_{L^{\infty}},\|\nabla \psi\|_{L^{\infty}},\||x| A \psi\|_{L^{\infty}}\right)>0$. The claim then follows by the equivalence $\left\|\nabla^{b} v\right\|_{L^{2}} \simeq\|\nabla v\|_{L^{2}}$ proved in Lemma 2.5, by complex interpolation and by density. 
Applying Lemma 4.1 we get

$$
\Im \int_{\Omega} a\left(\nabla \psi, \nabla^{b} u\right) u d x \leq\left|\int_{\Omega} a\left(\nabla \psi, \nabla^{b} u\right) u d x\right| \leq \widetilde{C}\|u\|_{\dot{H}^{\frac{1}{2}}}^{2}
$$

for some $\widetilde{C}$ depending on $\|a\|_{L^{\infty}},\|\nabla \psi\|_{L^{\infty}},\||x| A \psi\|_{L^{\infty}}$. Note that even if $\psi$ depends on $R>0$, the constant $\widetilde{C}$ does not, since by (4.5), (1.6),

$$
\|a \nabla \psi\|_{L^{\infty}} \leq \frac{1}{2}\|a\|_{L^{\infty}}, \quad\||x| A \psi\|_{L^{\infty}} \leq C\left(C_{a},\|a\|_{L^{\infty}}\right)
$$

4.8. Conclusion of the proof. From (3.1), using (4.40), we have

$$
\begin{aligned}
\partial_{t}\left[\Im\left(a\left(\nabla \psi, \nabla^{b} u\right) u\right)\right] \geq & -\frac{1}{2} A^{2} \psi|u|^{2}-\Re a(\nabla \psi, \nabla c)|u|^{2}+\Re\left(\alpha_{\ell m} \partial_{m}^{b} u \overline{\partial_{\ell}^{b} u}\right) \\
& +2 \Im\left(a_{j k} \partial_{k}^{b} u\left(\partial_{j} b_{\ell}-\partial_{\ell} b_{j}\right) a_{\ell m} \partial_{m} \psi \bar{u}\right) \\
& +\gamma_{0}[f(u) \bar{u}-2 F(u)](R \vee|x|)^{-1} \\
& +\partial_{j}\left\{-\Re Q_{j}+2 F(u) a_{j k} \partial_{k} \psi+\Im\left[u_{t} \bar{u} a_{j k} \partial_{k} \psi\right]\right\} .
\end{aligned}
$$

Integrating with respect to $t \in[0, T]$ and then $x \in \Omega$ we obtain

$$
\begin{aligned}
\int_{\Omega} \int_{0}^{T} \partial_{t} \Im & {\left[a\left(\nabla \psi, \nabla^{b} u\right) u\right] d t d x \geq } \\
& -\int_{\Omega} \int_{0}^{T}\left[\frac{1}{2} A^{2} \psi+\Re a(\nabla \psi, \nabla c)\right]|u|^{2} d t d x \\
& +\int_{\Omega} \int_{0}^{T} \Re\left[\alpha_{l m} \partial_{m}^{b} u \overline{\partial_{l}^{b} u}\right] d t d x \\
& +2 \int_{\Omega} \int_{0}^{T} \Im\left[a_{j k} \partial_{k}^{b} u\left(\partial_{j} b_{l}-\partial_{l} b_{j}\right) a_{l m} \partial_{m} \psi \bar{u}\right] d t d x \\
& +\gamma_{0} \int_{\Omega} \int_{0}^{T} \frac{f(u) \bar{u}-2 F(u)}{R \vee|x|} d t d x \\
& +\int_{\Omega} \int_{0}^{T} \partial_{j}\left\{-\Re Q_{j}+2 F(u) a_{j k} \partial_{k} \psi+\Im\left[u_{t} \bar{u} a_{j k} \partial_{k} \psi\right]\right\} d t d x
\end{aligned}
$$

We now use the estimates from the previous sections.

For the term (4.45), we use (4.44):

$$
\begin{aligned}
\int_{\Omega} \int_{0}^{T} & \partial_{t} \Im\left[a\left(\nabla \psi, \nabla^{b} u\right) u\right] d t d x \\
& \leq \int_{\Omega} \Im a\left(\nabla \psi, \nabla^{b} u(0)\right) u(0) d x+\int_{\Omega} \Im a\left(\nabla \psi, \nabla^{b} u(T)\right) u(T) d x \leq \\
& \leq C\left(\|u(0)\|_{\dot{H}^{\frac{1}{2}}}^{2}+\|u(T)\|_{\dot{H}^{\frac{1}{2}}}^{2}\right),
\end{aligned}
$$

where $C$ depends on $\|a\|_{L^{\infty}},\|\nabla \psi\|_{\infty},\||x| A \psi\|_{L^{\infty}}$, but not on $R>0$.

For (4.50) we swap the integrals, then using (4.42) we see that this term can be discarded.

4.8.1. Proof of Theorem 1.1. We estimate (4.47) using (4.35) and recalling that $\|\cdot\|_{Y} \leq\|\cdot\|_{\dot{Y}}$, while (4.48) is estimated using (4.38). Summing up, we have obtained

$$
\begin{aligned}
& C\left(\|u(0)\|_{\dot{H}^{\frac{1}{2}}}^{2}+\|u(T)\|_{\dot{H}^{\frac{1}{2}}}^{2}\right) \geq \\
& \quad \frac{1}{2}\left(\frac{n-1}{2} \nu \frac{1}{R^{2}} \int_{\Omega_{=R}} \widehat{a}\|u\|_{L_{T}^{2}} d S-\left[\frac{n+3}{2} N-n \nu\right](n-1) \int_{\Omega_{\geq R}} \widehat{a} \frac{R^{n-1}}{|x|^{n+2}}\|u\|_{L_{T}^{2}} d x\right) \\
& \quad-\left(12 n C_{a}\left(N+C_{a}\right)+C_{c}\right) \delta^{-1}\|u\|_{\dot{X}_{x} L_{T}^{2}}^{2} \\
& \quad+\frac{n-1}{n R} \nu^{2} \int_{\Omega_{\leq R}}\left\|\nabla^{b} u(x)\right\|_{L_{T}^{2}}^{2} d x-24 N C_{a} \delta^{-1}\left\|\nabla^{b} u\right\|_{\dot{Y}_{x} L_{T}^{2}}^{2} \\
& \quad-5 \delta^{-1} N^{2} C_{b}\left(\left\|\nabla^{b} u\right\|_{\dot{Y}_{x} L_{T}^{2}}^{2}+\|u\|_{\dot{X}_{x} L_{T}^{2}}^{2}\right) \\
& \quad+\gamma_{0} \int_{\Omega} \int_{0}^{T} \frac{f(u) \bar{u}-2 F(u)}{R \vee|x|} d t d x .
\end{aligned}
$$


We now take the sup over $R>0$ at the right hand side. Denote with $\Sigma(R)$ all the terms which depend on $R$ :

$$
\begin{aligned}
\Sigma(R):= & \frac{1}{2}\left(\frac{n-1}{2} \nu \frac{1}{R^{2}} \int_{\Omega_{=R}} \widehat{a}\|u\|_{L_{T}^{2}} d S\left[\frac{n+3}{2} N-n \nu\right](n-1) \int_{\Omega_{\geq R}} \widehat{a} \frac{R^{n-1}}{|x|^{n+2}}\|u\|_{L_{T}^{2}} d x\right) \\
& +\frac{n-1}{n R} \nu^{2} \int_{\Omega_{\leq R}}\left\|\nabla^{b} u(x)\right\|_{L_{T}^{2}}^{2} d x+\gamma_{0} \int_{\Omega} \int_{0}^{T} \frac{f(u) \bar{u}-2 F(u)}{R \vee|x|} d t d x
\end{aligned}
$$

We shall use the simple remark that if three nonnegative quantities $f, g, h$ depend on $R$, then

$$
\sup _{R>0}[f(R)+g(R)+h(R)] \geq \frac{1}{3}\left(\sup _{R>0} f(R)+\sup _{R>0} g(R)+\sup _{R>0} h(R)\right) .
$$

We now distinguish two cases.

First case: $\frac{n+3}{2} N \geq n \nu$. Then by (2.6) we get

$$
\Sigma(R) \geq Z(R)-\frac{1}{2}\left[\frac{n+3}{2} N-n \nu\right]\left\|\widehat{a}^{1 / 2} u\right\|_{\dot{X}_{x} L_{T}^{2}}^{2},
$$

where

$Z(R):=\frac{n-1}{4} \nu \frac{1}{R^{2}} \int_{\Omega_{=R}} \widehat{a}\|u\|_{L_{T}^{2}}^{2} d S+\frac{n-1}{n R} \nu^{2} \int_{\Omega_{\leq R}}\left\|\nabla^{b} u(x)\right\|_{L_{T}^{2}}^{2} d x+\gamma_{0} \int_{\Omega} \int_{0}^{T} \frac{f(u) \bar{u}-2 F(u)}{R \vee|x|} d t d x$.

Thanks to (2.6), (4.52), and recalling that $\widehat{a} \geq \nu$, we obtain

$\sup _{R>0} Z(R) \geq \frac{n-1}{12} \nu^{2}\|u\|_{\dot{X}_{x} L_{T}^{2}}^{2}+\frac{n-1}{3 n} \nu^{2}\left\|\nabla^{b} u\right\|_{\dot{Y}_{x} L_{T}^{2}}^{2}+\frac{\gamma_{0}}{3} \int_{\Omega} \int_{0}^{T} \frac{f(u(x)) \bar{u}(x)-2 F(u)}{|x|} d t d x$ and consequently, again by $\widehat{a} \geq \nu$,

$\sup _{R>0} \Sigma(R) \geq \frac{K_{0}}{2} \nu^{2}\|u\|_{\dot{X}_{x} L_{T}^{2}}^{2}+\frac{n-1}{3 n} \nu^{2}\left\|\nabla^{b} u\right\|_{\dot{Y}_{x} L_{T}^{2}}^{2}+\frac{\gamma_{0}}{3} \int_{\Omega} \int_{0}^{T} \frac{f(u(x)) \bar{u}(x)-2 F(u)}{|x|} d t d x$

provided we define

$$
K_{0}:=\frac{n-1}{6}-\frac{n+3}{2} \frac{N}{\nu}+n
$$

which is a strictly positive quantity provided we assume $N / \nu$ is small enough (like in (4.2)).

Second case: $\frac{n+3}{2} N \leq n \nu$. Then we have

$\Sigma(R) \geq \frac{n-1}{4} \nu \frac{1}{R^{2}} \int_{\Omega_{=R}} \widehat{a}\|u\|_{L_{T}^{2}}^{2} d S+\frac{n-1}{n R} \nu^{2} \int_{\Omega_{\leq R}}\left\|\nabla^{b} u(x)\right\|_{L_{T}^{2}}^{2} d x+\gamma \int_{\Omega} \int_{0}^{T} \frac{f(u) \bar{u}-2 F(u)}{R \vee|x|} d t d x$.

Thanks to (4.52), recalling that $\widehat{a} \geq \nu$, and observing that in this case $K_{0} \leq \frac{n-1}{6}$, we obtain again (4.53).

By (4.51), (4.53) we conclude that

$$
M_{1}\|u\|_{\dot{X}_{x} L_{T}^{2}}^{2}+M_{2}\left\|\nabla^{b} u\right\|_{\dot{Y}_{x} L_{T}^{2}}^{2}+M_{3} \int_{\Omega} \int_{0}^{T} \frac{f(u(x)) \bar{u}(x)-2 F(u)}{|x|} d t d x \leq C\left(\|u(0)\|_{\dot{H}^{\frac{1}{2}}}^{2}+\|u(T)\|_{\dot{H}^{\frac{1}{2}}}^{2}\right)
$$

for some $C>0$, where $\gamma_{0}$ is defined in (4.41) and

$$
\begin{gathered}
M_{1}:=\frac{K_{0}}{2} \nu^{2}-\frac{5 N^{2} C_{b}+12 n C_{a}\left(N+C_{a}\right)+C_{c}}{\delta}, \\
M_{2}:=\frac{n-1}{3 n} \nu^{2}-\frac{5 N^{2} C_{b}+24 N C_{a}}{\delta}, \quad M_{3}:=\frac{\gamma_{0}}{3} .
\end{gathered}
$$

If the constants $C_{a}, C_{b}$ and $C_{c}$ are sufficiently small, these quantities are positive, and the estimate (4.56) is effective. 
4.8.2. Proof of Theorem 1.2. We estimate (4.47) using (4.35) and (4.48) thanks to (4.39). Summing up, we have obtained

$$
\begin{aligned}
C\left(\|u(0)\|_{\dot{H}^{\frac{1}{2}}}^{2}\right. & \left.+\|u(T)\|_{\dot{H}^{\frac{1}{2}}}^{2}\right) \geq \\
& \left(1-C_{I}\right)^{2} \frac{1}{R^{2}} \int_{\Omega_{=R}}\|u\|_{L_{T}^{2}}^{2} d S \\
& -8 \delta^{-1}\left[C_{c}+9 C_{I}+41 C_{a}\left(N+C_{a}\right)\right]\|u\|_{X L_{T}^{2}}^{2} \\
& -13 \delta^{-1}\left[C_{c}+36 C_{a}\left(N+C_{a}\right)\right]\left\|\nabla^{b} u\right\|_{Y L_{T}^{2}}^{2} \\
& +\frac{n-1}{n R} \nu^{2} \int_{\Omega_{\leq R}}\left\|\nabla^{b} u(x)\right\|_{L_{T}^{2}}^{2} d x-24 N C_{a} \delta^{-1}\left\|\nabla^{b} u\right\|_{Y_{x} L_{T}^{2}}^{2} \\
& -9 \delta^{-1} N^{2} C_{b}\left(\left\|\nabla^{b} u\right\|_{Y_{x} L_{T}^{2}}^{2}+\|u\|_{X_{x} L_{T}^{2}}^{2}\right) \\
& +\gamma_{0} \int_{\Omega} \int_{0}^{T} \frac{f(u) \bar{u}-2 F(u)}{R \vee|x|} d t d x .
\end{aligned}
$$

We now take the sup over $R>1$ at the right hand side. We denote with $\Sigma(R)$ all the terms which depend on $R$ :

$$
\begin{aligned}
\Sigma(R):= & \left(1-C_{I}\right)^{2} \frac{1}{R^{2}} \int_{\Omega_{=R}}\|u\|_{L_{T}^{2}}^{2} d S+\frac{n-1}{n R} \nu^{2} \int_{\Omega_{\leq R}}\left\|\nabla^{b} u(x)\right\|_{L_{T}^{2}}^{2} d x \\
& +\gamma \int_{\Omega} \int_{0}^{T} \frac{f(u) \bar{u}-2 F(u)}{R \vee|x|} d t d x
\end{aligned}
$$

Thanks to (2.14), we have, for $0<\theta<1$,

$$
\begin{aligned}
\left(1-C_{I}\right)^{2} \sup _{R>1} \frac{1}{R^{2}} \int_{\Omega_{=R}}\|u\|_{L_{T}^{2}}^{2} d S \geq & (1-\theta)\left(1-C_{I}\right)^{2} \sup _{R>1} \frac{1}{R^{2}} \int_{\Omega_{=R}}\|u\|_{L_{T}^{2}}^{2} d S \\
& +\theta\left(1-C_{I}\right)^{2}\left(\frac{1}{4}\|u\|_{X L_{T}^{2}}^{2}-\frac{13}{4}\left\|\nabla^{b} u\right\|_{Y L_{T}^{2}}^{2}\right) .
\end{aligned}
$$

Note also that we can take $\nu=1-C_{I}$ and $N=1+C_{I}$ by assumption (1.16), while $n=3$. We obtain

$$
\sup _{R>1} \frac{n-1}{n R} \nu^{2} \int_{\Omega_{\leq R}}\left\|\nabla^{b} u(x)\right\|_{L_{T}^{2}}^{2} d x \geq \frac{2}{3}\left(1-C_{I}\right)^{2}\left\|\nabla^{b} u\right\|_{Y L_{T}^{2}}^{2}
$$

Finally

$$
\gamma_{0} \sup _{R>1} \int_{\Omega} \int_{0}^{T} \frac{f(u) \bar{u}-2 F(u)}{R \vee|x|} d t d x \geq \gamma_{0} \int_{\Omega} \int_{0}^{T} \frac{f(u) \bar{u}-2 F(u)}{\langle x\rangle} d t d x .
$$

We take $\theta:=2 / 13$ (it is enough to choose $\theta$ such that $2 / 3 \geq(13 \theta) / 4$ ). Thanks to (4.52), (4.61), (4.62), (4.63), we get

$$
\begin{aligned}
\sup _{R>1} \Sigma(R) \geq & \frac{\left(1-C_{I}\right)^{2}}{78}\|u\|_{X L_{T}^{2}}^{2}+\frac{\left(1-C_{I}\right)^{2}}{6}\left\|\nabla^{b} u\right\|_{Y L_{T}^{2}}^{2} \\
& +\frac{\gamma_{0}}{3} \int_{\Omega} \int_{0}^{T} \frac{f(u) \bar{u}-2 F(u)}{\langle x\rangle} d t d x .
\end{aligned}
$$

By (4.60), (4.64) we conclude that

$$
\begin{aligned}
M_{1}\|u\|_{X_{x} L_{T}^{2}}^{2}+M_{2}\left\|\nabla^{b} u\right\|_{Y_{x} L_{T}^{2}}^{2}+M_{3} \int_{\Omega} \int_{0}^{T} \frac{f(u(x)) \bar{u}(x)-2 F(u)}{\langle x\rangle} d t d x \\
\leq C\left(\|u(0)\|_{\dot{H}^{\frac{1}{2}}}^{2}+\|u(T)\|_{\dot{H}^{\frac{1}{2}}}^{2}\right)
\end{aligned}
$$


for some $C>0$, where

$$
\begin{aligned}
& M_{1}^{\prime}:=\frac{\left(1-C_{I}\right)^{2}}{78}-8 \delta^{-1}\left[C_{c}+9 C_{I}+41 C_{a}\left(N+C_{a}\right)\right]-9 \delta^{-1} N^{2} C_{b}, \\
& M_{2}^{\prime}:=\frac{\left(1-C_{I}\right)^{2}}{6}-13 \delta^{-1}\left[C_{c}+38 C_{a}\left(N+C_{a}\right)\right]-9 \delta^{-1} N^{2} C_{b}, \\
& M_{3}=\frac{\gamma_{0}}{3},
\end{aligned}
$$

and $\gamma_{0}$ is defined in (4.41). If the constants $C_{a}, C_{b}, C_{c}$ and $C_{I}$ are sufficiently small, these quantities are positive and the estimate (4.65) is effective.

4.9. Proof of Corollary 1.3. Since $u=e^{i t L} u_{0}$ satisfies equation (1.1) with the choice $f \equiv 0$, we see that $u$ satisfies the smoothing estimate (1.20). By complex interpolation, we deduce from (1.20) the estimate

$$
\left\|\langle x\rangle^{-1-}(-\Delta)^{\frac{1}{4}} u\right\|_{L_{T}^{2} L^{2}} \lesssim\left\|u_{0}\right\|_{\dot{H}^{\frac{1}{2}}}+\|u(T)\|_{\dot{H}^{\frac{1}{2}}}
$$

for all $T>0$. Proceeding exactly as in the proof of Corollary 1.4 in [4], from the gaussian bound for $e^{t L}$ in Proposition 6.2 we deduce the weighted estimate

$$
\left\|w(x)(-L)^{\frac{1}{4}} v\right\|_{L^{2}} \lesssim\left\|w(x)(-\Delta)^{\frac{1}{4}} v\right\|_{L^{2}}
$$

for any $A_{2}$ weight $w$, and in particular for $w(x)=\langle x\rangle^{-s}$ for any $s>0$. Thus we have

$$
\left\|\langle x\rangle^{-1-}(-L)^{\frac{1}{4}} u\right\|_{L_{T}^{2} L^{2}} \lesssim\left\|\langle x\rangle^{-1-}(-\Delta)^{\frac{1}{4}} u\right\|_{L_{T}^{2} L^{2}} \lesssim\left\|u_{0}\right\|_{\dot{H}^{\frac{1}{2}}}+\|u(T)\|_{\dot{H}^{\frac{1}{2}}}
$$

and commuting $(-L)^{\frac{1}{4}}$ with $e^{i t L}$, and recalling the equivalence (6.2), we obtain

$$
\left\|\langle x\rangle^{-1-} u\right\|_{L_{T}^{2} L^{2}} \lesssim\left\|u_{0}\right\|_{L^{2}}+\|u(T)\|_{L^{2}} \simeq\left\|u_{0}\right\|_{L^{2}}
$$

by the conservation of the $L^{2}$ norm.

\section{Proof of Theorems 1.4, 1.5: the Bilinear Smoothing estimate}

Since the arguments for Theorems 1.4 and 1.5 largely overlap, we shall again proceed with both proofs in parallel.

Let $u$ be a solution of (1.1), and write identity (3.1) with a weight of the form $\psi=\psi(x-y)$, for $x, y \in \Omega$. In the following formulas, to make notations lighter, we shall write simply $u(x), u(y)$ instead of $u(t, x), u(t, y)$. We have

$$
\begin{aligned}
& M(x, y)=-\frac{1}{2} A_{x}^{2} \psi(x-y)|u(x)|^{2}+\Re\left(\alpha_{\ell m}(x) \partial_{x_{m}}^{b(x)} u(x) \overline{\partial_{x_{\ell}}^{b(x)} u(x)}\right) \\
& \quad-\Re a(x)\left(\nabla_{x} \psi(x-y), \nabla_{x} c(x)\right)|u(x)|^{2} \\
& \quad+2 \Im\left(a_{j k}(x) \partial_{x_{k}}^{b(x)} u(x)\left(\partial_{x_{j}} b(x)_{\ell}-\partial_{x_{\ell}} b(x){ }_{j}\right) a_{\ell m}(x) \partial_{x_{m}} \psi(x-y) \overline{u(x)}\right) \\
& \quad+A_{x} \psi(x-y)[f(u(x)) \bar{u}(x)-2 F(u(x))] \\
& \quad+\partial_{x_{j}}\left\{-\Re Q_{j}(x)+2 F(u(x)) a_{j k}(x) \partial_{x_{k}} \psi(x-y)+\Im\left[u_{t}(x) \bar{u}(x) a_{j k}(x) \partial_{x_{k}} \psi(x-y)\right]\right\} .
\end{aligned}
$$

where $M(x, y)$ is defined by

$$
M(x, y):=\partial_{t}\left\{\Im\left(a_{x}\left(\nabla_{x} \psi(x-y), \nabla_{x}^{b(x)} u(x)\right) u(x)\right)\right\} .
$$

Note that in order to distinguish between the two groups of variables $x$ and $y$, here and in the following we used the notations

$a(x)(z, w)=a_{j k}(x) z_{j} \overline{w_{k}}, \quad \partial_{x_{j}}^{b(x)}=\partial_{x_{j}}+i b_{j}(x), \quad A_{x}^{b(x)} v=\partial_{x_{j}}^{b(x)}\left(a_{j k}(x) \partial_{x_{k}}^{b(x)} v(x, y)\right)$ 
and similarly $A_{x}, \nabla_{x}^{b(x)}$; we shall however stick to simpler notations whenever possible. The starting point for the proof is the identity

$$
\begin{aligned}
& \partial_{t}\left\{\Im\left[a(x)\left(\nabla_{x} \psi(x-y), \nabla^{b} u(x)\right) u(x)\right]|u(y)|^{2}\right\}= \\
& \quad=M(x, y)|u(y)|^{2}+\Im\left[a(x)\left(\nabla \psi(x-y), \nabla^{b} u(x)\right) u(x)\right] \partial_{t}\left\{|u(y)|^{2}\right\} .
\end{aligned}
$$

Since $u$ is a solution of (1.1) and $c, f(u) \bar{u}$ are real valued, we have

$$
\begin{aligned}
\partial_{t}|u|^{2} & =2 \Re\left[u_{t} \bar{u}\right]=2 \Re\left[\bar{u}\left(-i A^{b} u+i c u+i f(u)\right)\right]= \\
& =2 \Re\left[-i A^{b} u \bar{u}+i c|u|^{2}+i f(u) \bar{u}\right]=2 \Im\left[A^{b} u \bar{u}\right]
\end{aligned}
$$

and using the identity

$$
A^{b} u \bar{u}=-a\left(\nabla^{b} u, \nabla^{b} u\right)+\nabla \cdot\left\{a \nabla^{b} u \bar{u}\right\},
$$

by the reality of $a\left(\nabla^{b} u, \nabla^{b} u\right)$ we have

$$
\partial_{t}|u(y)|^{2}=2 \Im\left[A^{b(y)} u(y) \overline{u(y)}\right]=2 \nabla_{y} \cdot\left\{\Im\left[a(y) \nabla_{y}^{b(y)} u(y) \bar{u}(y)\right]\right\} .
$$

Thus the last term in (5.1) can be manipulated as follows:

$$
\begin{aligned}
\Im[a(x) & \left.\left(\nabla \psi(x-y), \nabla^{b} u(x)\right) u(x)\right] \partial_{t}\left[|u(y)|^{2}\right]= \\
= & 2 \Im\left[a(x)\left(\nabla \psi(x-y), \nabla^{b} u(x)\right) u(x)\right] \nabla_{y} \cdot\left\{\Im\left[a(y) \nabla^{b(y)} u(y) \bar{u}(y)\right]\right\}= \\
= & 2 \Im\left[\left(a(x) \overline{\nabla_{x}^{b} u(x)}\right)^{t} u(x)\right] D^{2} \psi(x-y) \Im\left[\left(a(y) \nabla_{y}^{b} u(y)\right) \overline{u(y)}\right] \\
& +\nabla_{y} \cdot\left\{2 \Im\left[a(x)\left(\nabla \psi(x-y), \nabla^{b} u(x)\right) u(x)\right] \Im\left[a(y) \nabla^{b(y)} u(y) \bar{u}(y)\right]\right\} .
\end{aligned}
$$

Moreover, we rewrite the quantities $\alpha_{\ell m}$ in the form

$$
\alpha_{\ell m}=2\left(a(x) D_{x}^{2} \psi(x-y) a(x)\right)_{\ell m}+r_{\ell m}
$$

where the first term is the $\ell m$ entry of the matrix $a \cdot D^{2} \psi \cdot a$ and

$$
r_{l m}=\partial_{k} \psi_{y}\left(2 a_{j m} a_{l k ; j}-a_{j k} a_{l m ; j}\right) .
$$

We choose different weights for the proofs of Theorem 1.4 and Theorem 1.5: in the proof of Theorem 1.5 we set

$$
\psi(x-y):=\langle x-y\rangle_{\sigma},
$$

for $\sigma>0$, where we use the following notation:

$$
\langle x-y\rangle_{\sigma}:=\left(\sigma^{2}+|x-y|^{2}\right)^{1 / 2},
$$

while in the proof of Theorem 1.4 we take $\sigma=0$ in (5.3), that is to say, we choose

$$
\psi(x-y):=|x-y|,
$$

Note that in the following, with a small abuse, we shall use the same notation for the radial weight function $\psi(x)$ and for $\psi(r)=\psi(|x|)$. We gather here some identities satisfied by $\psi(r)=\langle r\rangle_{\sigma}$ for $\sigma \geq 0$ :

$$
\begin{gathered}
\psi^{\prime}=\frac{r}{\langle r\rangle_{\sigma}}, \quad \psi^{\prime \prime}=\frac{\sigma^{2}}{\langle r\rangle_{\sigma}^{3}}, \quad \psi^{\prime \prime \prime}=-3 \sigma^{2} \frac{r}{\langle r\rangle_{\sigma}^{5}}, \quad \psi^{I V}=12 \frac{\sigma^{2}}{\langle r\rangle_{\sigma}^{5}}-15 \frac{\sigma^{4}}{\langle r\rangle_{\sigma}^{7}}, \\
\frac{1}{r^{2}}\left(\psi^{\prime \prime}-\frac{\psi^{\prime}}{r}\right)=-\frac{1}{\langle r\rangle_{\sigma}^{3}}, \quad \psi^{\prime \prime \prime}-\frac{\psi^{\prime \prime}}{r}=-\sigma^{2}\left(\frac{r}{\langle r\rangle_{\sigma}^{5}}+\frac{1}{\langle r\rangle_{\sigma}^{3} r}\right) \leq \frac{4 \sigma^{2}}{\langle r\rangle_{\sigma}^{3} r} .
\end{gathered}
$$

Moreover, for $\sigma \geq 0$, we introduce the notation

$$
\widehat{(x-y)_{m}^{\sigma}}{ }^{\sigma}=\frac{x_{m}-y_{m}}{\langle x-y\rangle_{\sigma}} .
$$


We have (see (4.10))

$$
\begin{aligned}
A_{x}\langle x-y\rangle_{\sigma}= & a_{\ell m ; \ell}(x)(\widehat{x-y})_{m}^{\sigma}+ \\
& +\frac{\sigma^{2}}{\langle x-y\rangle_{\sigma}} a_{\ell m}(x)(\widehat{x-y})_{\ell}^{\sigma}(\widehat{x-y})_{m}^{\sigma}+\frac{\bar{a}(x)-(\widehat{x-y})_{\ell}^{\sigma} a_{\ell m}(x)(\widehat{x-y})_{m}^{\sigma}}{\langle x-y\rangle_{\sigma}}
\end{aligned}
$$

which implies, since the last two terms are non negative,

$$
A_{x}\langle x-y\rangle_{\sigma} \geq a_{\ell m ; \ell}(x) \widehat{(x-y)_{m}^{\sigma}} \geq-\left|a^{\prime}(x)\right| \geq-\frac{C_{a}}{\langle x\rangle^{1+\delta}},
$$

and, using assumption (1.13),

$A_{x}\langle x-y\rangle_{\sigma}[f(u(x)) \overline{u(x)}-2 F(u(x))]|u(y)|^{2} \geq-\frac{C_{a}}{\langle x\rangle^{1+\delta}}[f(u(x)) \overline{u(x)}-2 F(u(x))]|u(y)|^{2}$.

Now we integrate (5.1) on $\Omega^{2}=\Omega_{x} \times \Omega_{y}$. The divergence terms in $\nabla_{x}$ can be neglected exactly as in the proof of Theorems 1.1 and 1.2, while the divergence terms in $\nabla_{y}$ vanish on $\partial \Omega_{y}$ and at infinity. Taking into account the previous computations we obtain the inequality

$$
\begin{aligned}
& 2 \int_{\Omega^{2}} \Re\left[\left(a(x) \overline{\nabla^{b} u(x)}\right)^{t} D^{2} \psi(x-y)\left(a(x) \nabla^{b} u(x)\right)\right]|u(y)|^{2} d x d y+ \\
& \quad+2 \int_{\Omega^{2}} \Im\left[\left(a(x) \overline{\nabla^{b} u(x)}\right)^{t} u(x)\right] D^{2} \psi(x-y) \Im\left[\left(a(y) \nabla^{b} u(y)\right) \overline{u(y)}\right] d x d y+ \\
& \quad-\frac{1}{2} \int_{\Omega^{2}} A^{2} \psi(x-y)|u(x)|^{2}|u(y)|^{2} d x d y \leq \\
& \leq \partial_{t} \int_{\Omega^{2}} \Im\left[a(x)\left(\nabla \psi(x-y), \nabla^{b} u(x)\right) u(x)\right]|u(y)|^{2} d x d y+\int_{\Omega^{2}} R(x, y)|u(y)|^{2} d x d y,
\end{aligned}
$$

where

$$
\begin{aligned}
R(x, y)= & -r_{l m}(x) \partial_{m}^{b} u(x) \overline{\partial_{l}^{b} u(x)} \\
& +\Re[a(x)(\nabla \psi(x-y), \nabla c(x))]|u(x)|^{2} \\
& -2 \Im\left[a_{j k}(x) \partial_{k}^{b(x)} u(x)\left(\partial_{j} b_{\ell}(x)-\partial_{\ell} b_{j}(x)\right) a_{\ell m}(x) \partial_{m} \psi(x-y) \overline{u(x)}\right] \\
& -C_{a}\langle x\rangle^{-1-\delta}[f(u(x)) \overline{u(x)}-2 F(u(x))]|u(y)|^{2} .
\end{aligned}
$$

We remark that $R(x, y)$ depends on $y$ only through $\psi$. In the following sections we integrate (5.5) in time on an interval $[0, T]$ and we estimate each term individually.

5.1. Positivity of quadratic terms in the derivative. The first two terms in (5.5) can be dropped from the inequality since their sum is nonnegative. To check this fact, we rewrite them as the sum

$$
\begin{aligned}
& \int_{\Omega^{2}}\left(a(x) \overline{\nabla^{b} u(x)}\right)^{t} D^{2} \psi(x-y)\left(a(x) \nabla^{b} u(x)\right)|u(y)|^{2} d x d y \\
& \quad+\int_{\Omega^{2}}\left(a(y) \overline{\nabla^{b} u(y)}\right)^{t} D^{2} \psi(x-y)\left(a(y) \nabla^{b} u(y)\right)|u(x)|^{2} d x d y \\
& \quad+2 \int_{\Omega^{2}} \Im\left[\left(a(x) \overline{\nabla^{b} u(x)}\right)^{t} u(x)\right] D^{2} \psi(x-y) \Im\left[\left(a(y) \nabla^{b} u(y)\right) \overline{u(y)}\right] d x d y
\end{aligned}
$$

and then positivity follows from the the following algebraic lemma with the choice $T(x, y)=D_{x}^{2} \psi(x-y)$ :

Lemma 5.1. Let $T(x, y)$ be a real, symmetric, nonnegative matrix depending on $x, y \in \mathbb{R}^{n}$. Then the following quantity is nonnegative a.e.:

$$
\begin{gathered}
\left(a(x){\overline{\nabla^{b} u(x)}}^{t} T(x, y)\left(a(x) \nabla^{b} u(x)\right)|u(y)|^{2}+\left(a(y) \overline{\nabla^{b} u(y)}\right)^{t} T(x, y)\left(a(y) \nabla^{b} u(y)\right)|u(x)|^{2}\right. \\
+2 \Im\left[\left(a(x) \overline{\nabla^{b} u(x)}\right)^{t} u(x)\right] T(x, y) \Im\left[\left(a(y) \nabla^{b} u(y)\right) \overline{u(y)}\right] \geq 0 .
\end{gathered}
$$


Proof. Let $\Sigma(x, y)$ be the quantity in the statement. Assume first $T=\operatorname{diag}\left(\lambda_{1}, \ldots, \lambda_{n}\right)$ is diagonal at a point $(x, y)$, with $\lambda_{j} \geq 0$. We have then

$$
\begin{gathered}
\Sigma(x, y)=\sum_{j=1}^{n} \lambda_{j}\left\{\left|\left(a(x) \nabla^{b} u(x)\right)_{j}\right|^{2}|u(y)|^{2}+\left|\left(a(y) \nabla^{b} u(y)\right)_{j}\right|^{2}|u(x)|^{2}\right. \\
\left.+2 \Im\left[\left(a(x) \overline{\nabla^{b} u(x)}\right)_{j} u(x)\right] \Im\left[\left(a(y) \nabla^{b} u(y)\right)_{j} \overline{u(y)}\right]\right\} \\
\geq \sum_{j=1}^{n} \lambda_{j}\left\{\left|\left(a(x) \nabla^{b} u(x)\right)_{j}\right|^{2}|u(y)|^{2}+\left|\left(a(y) \nabla^{b} u(y)\right)_{j}\right|^{2}|u(x)|^{2}\right. \\
\left.-2\left|\left(a(x) \overline{\nabla^{b} u(x)}\right)_{j}\right||u(x)|\left|\left(a(y) \nabla^{b} u(y)\right)_{j}\right||\overline{u(y)}|\right\} \geq 0 .
\end{gathered}
$$

If $T(x, y)$ is non diagonal, we can find an orthonormal matrix $S=S(x, y)$ with real entries such that $T=S^{t} \Lambda S$, with $\Lambda \geq 0$ real and diagonal. This implies

$$
\begin{aligned}
\Sigma(x, y)= & \left(S a(x) \overline{\nabla^{b} u(x)}\right)^{t} \Lambda\left(S a(x) \nabla^{b} u(x)\right)|u(y)|^{2} \\
& +\left(S a(y) \overline{\nabla^{b} u(y)}\right)^{t} \Lambda\left(S a(y) \nabla^{b} u(y)\right)|u(x)|^{2} \\
& +2 \Im\left[\left(S a(x) \overline{\nabla^{b} u(x)}\right)^{t} u(x)\right] \Lambda \Im\left[\left(S a(y) \nabla^{b} u(y)\right) \overline{u(y)}\right],
\end{aligned}
$$

and the claim follows from the previous step.

5.2. The $\partial_{t}$ term. We now consider the first term at the right hand side of $(5.5)$, which is differentiated in time. We need a Lemma:

Lemma 5.2. Let $\psi(x-y)=\langle x-y\rangle_{\sigma}$, for $\sigma \geq 0$. Then the following estimate holds:

$$
\left|\int_{\Omega^{2}} a(x)\left(\nabla \psi(x-y), \nabla^{b} u(x)\right) u(x) \varphi(y) d x d y\right| \lesssim\|\varphi\|_{L^{1}}\|u\|_{\dot{H}^{\frac{1}{2}}}^{2},
$$

for an implicit constant independent on $\sigma$.

Proof. For $f, g \in C_{c}^{\infty}(\Omega)$, set

$$
T(f, g):=\int_{\Omega^{2}} a(x)\left(\nabla \psi(x-y), \nabla^{b} f(x)\right) g(x) \varphi(y) d x d y .
$$

We have immediately

$$
|T(f, g)| \leq\|a\|_{L^{\infty}}\|\varphi\|_{L^{1}}\left\|\nabla^{b} f\right\|_{L^{2}}\|g\|_{L^{2}} .
$$

On the other hand, integrating by parts we get

$$
\begin{aligned}
|T(f, g)| \leq & \left|\int_{\Omega^{2}} a(x)\left(\nabla \psi(x-y), \nabla^{b} g(x)\right) f(x) \overline{\varphi(y)} d x d y\right| \\
& +\left|\int_{\Omega^{2}} \partial_{x_{m}} a_{\ell m}(x) \partial_{x_{\ell}} \psi(x-y) \overline{f(x)} g(x) \varphi(y) d x d y\right| \\
& +\left|\int_{\Omega^{2}} a_{\ell m}(x) \partial_{x_{\ell} x_{m}} \psi(x-y) \overline{f(x)} g(x) \varphi(y) d y d x\right| .
\end{aligned}
$$

By assumption (1.6), we have

$$
\begin{aligned}
& \left|\int_{\Omega^{2}} \partial_{x_{m}} a_{\ell m}(x) \partial_{x_{\ell}} \psi(x-y) \overline{f(x)} g(x) \varphi(y) d x d y\right| \leq \\
& \leq C_{a}\|\varphi\|_{L^{1}}\|f\|_{L^{2}}\left\|\langle x\rangle^{-1-\delta} g\right\|_{L^{2}} \lesssim\|\varphi\|_{L^{1}}\|f\|_{L^{2}}\left\|\nabla^{b} g\right\|_{L^{2}}
\end{aligned}
$$

where in the last step we used (2.11). By direct computation

$$
\begin{aligned}
\mid \int_{\Omega^{2}} a_{\ell m}(x) & \partial_{x_{\ell} x_{m}} \psi(x-y) \overline{f(x)} g(x) \varphi(y) d y d x \mid \\
& \leq \int_{\mathbb{R}^{2 n}}\left|\bar{a}(x)-a(x)\left(\widehat{(x-y)^{\sigma}},(\widehat{(x-y)})^{\sigma}\right)\right| \cdot\langle x-y\rangle_{\sigma}^{-1} \cdot|\overline{f(x)} g(x) \varphi(y)| d x d y \\
& \leq N n\|\varphi\|_{L^{1}}\|f\|_{L^{2}} \sup _{y}\left(\int_{\mathbb{R}^{n}} \frac{|g(x)|^{2}}{|x-y|^{2}} d x\right)^{\frac{1}{2}} \\
& \lesssim\|\varphi\|_{L^{1}}\|f\|_{L^{2}}\left\|\nabla^{b} g\right\|_{L^{2}},
\end{aligned}
$$

again using (2.11) in the last inequality. By (5.9) and (5.10), we deduce from (5.8)

$$
|T(f, g)| \lesssim\|\varphi\|_{L^{1}}\|f\|_{L^{2}}\left\|\nabla^{b} g\right\|_{L^{2}} .
$$


Recalling now the equivalence (2.15), by complex interpolation beetwen this estimate and (5.7) we obtain

$$
\left|\int_{\mathbb{R}^{n}} a(x)\left(\nabla \psi(x-y), \nabla^{b} f(x)\right) g(x) \varphi(y) d x d y\right| \lesssim\|\varphi\|_{L^{1}}\|f\|_{\dot{H}^{\frac{1}{2}}}\|g\|_{\dot{H}^{\frac{1}{2}}}
$$

and taking $f=g=u$ we conclude the proof.

If we choose $\varphi=|u|^{2}$ in the previous Lemma, we obtain

$$
\begin{gathered}
\left.\left|\int_{0}^{T} \partial_{t} \int_{\Omega^{2}} \Im\left[a(x)\left(\nabla \psi(x-y), \nabla^{b} u(x)\right) u(x)\right]\right| u(y)\right|^{2} d x d y d t \mid \\
\lesssim\|u(0)\|_{L^{2}}^{2}\left[\|u(0)\|_{\dot{H}^{\frac{1}{2}}}^{2}+\|u(T)\|_{\dot{H}^{\frac{1}{2}}}^{2}\right]
\end{gathered}
$$

since the $L^{2}$-norm of the solution is constant in time.

5.3. The $R(x, y)$ term. We now focus on the last term in (5.5). Our goal is to prove

$$
\left.\left|\int_{0}^{T} \int_{\Omega^{2}} R(x, y)\right| u(y)\right|^{2} d x d y d t \mid \lesssim\|u(0)\|_{L^{2}}^{2}\left[\|u(0)\|_{\dot{H}^{\frac{1}{2}}}^{2}+\|u(T)\|_{\dot{H}^{\frac{1}{2}}}^{2}\right] .
$$

The quantity $R(x, y)$, defined by (5.6), gives rise to four terms.

For the term containing $r_{\ell m}$ (see (5.2)) we notice that for all $\sigma \geq 0$ we have $|\nabla \psi| \leq 1$, hence both in the proof of Theorem 1.4 and Theorem 1.5 we have

$$
\left|r_{\ell m}(x)\right| \leq 2 N\left|a^{\prime}(x)\right| \leq 2 N C_{a}\langle x\rangle^{-1-\delta}
$$

using (1.6). This implies

$$
\left.\left|\int_{0}^{T} \int_{\Omega^{2}} r_{\ell m}(x) \partial_{m}^{b} u(x) \overline{\partial_{\ell}^{b} u(x)}\right| u(y)\right|^{2} d x d y d t \mid \lesssim\|u(0)\|_{L^{2}}^{2} \int_{\mathbb{R}^{n}}\langle x\rangle^{-1-\delta}\left\|\nabla^{b} u(x)\right\|_{L_{t}^{2}}^{2} d x
$$

by the conservation of the $L^{2}$ norm. In the proof of Theorem 1.4, by estimate (2.9) and (1.15) we obtain

$$
\left.\left|\int_{0}^{T} \int_{\Omega^{2}} r_{\ell m}(x) \partial_{m}^{b} u(x) \overline{\partial_{\ell}^{b} u(x)}\right| u(y)\right|^{2} d x d y d t \mid \lesssim\|u(0)\|_{L^{2}}^{2}\left[\|u(0)\|_{\dot{H}^{\frac{1}{2}}}^{2}+\|u(T)\|_{\dot{H}^{\frac{1}{2}}}^{2}\right],
$$

and in the proof of Theorem 1.5 we get the same result thanks to (2.9) and (1.18).

We estimate differently the term containing $c$ in the two proofs. In the proof of Theorem 1.4, recalling assumption (1.22), we have

$$
\begin{aligned}
\mid \int_{0}^{T} \int_{\Omega^{2}} a(x) & (\nabla \psi(x-y), \nabla c(x))|u(x)|^{2}|u(y)|^{2} d x d y d t \mid \\
& \lesssim\|u(0)\|_{L^{2}} \int_{\Omega}\|u(x)\|_{L_{t}^{2}}^{2}|x|^{-2}\langle x\rangle^{-1-\delta} d x \lesssim\|u(0)\|_{L_{x}^{2}}^{2}\|u\|_{\dot{X} L_{t}^{2}}^{2}
\end{aligned}
$$

using the inequality (2.7), and, thanks to (1.15),

$\left.\left|\int_{0}^{T} \int_{\Omega^{2}} a(x)(\nabla \psi(x-y), \nabla c)\right| u(x)\right|^{2}|u(y)|^{2} d x d y d t \mid \lesssim\|u(0)\|_{L^{2}}^{2}\left[\|u(0)\|_{\dot{H}^{\frac{1}{2}}}^{2}+\|u(T)\|_{\dot{H}^{\frac{1}{2}}}^{2}\right]$.

In the proof of Theorem 1.5, recalling assumption (1.22) and thanks to (4.32), we have

$$
\begin{aligned}
\mid \int_{0}^{T} \int_{\Omega^{2}} a(x) & (\nabla \psi(x-y), \nabla c(x))|u(x)|^{2}|u(y)|^{2} d x d y d t \mid \\
& \lesssim\|u(0)\|_{L^{2}} \int_{0}^{T} \int_{\Omega}|x|^{-2}\langle x\rangle^{-1-\delta}|u(x)|^{2} d x d t \\
& \lesssim\|u(0)\|_{L^{2}}\left[\|u\|_{X L_{T}^{2}}^{2}+\left\|\nabla^{b} u\right\|_{Y L_{T}^{2}}^{2}\right] \\
& \lesssim\|u(0)\|_{L^{2}}\left[\|u(0)\|_{\dot{H}^{\frac{1}{2}}}^{2}+\|u(T)\|_{\dot{H}^{\frac{1}{2}}}^{2}\right] .
\end{aligned}
$$

We turn to the estimate of the term containing $b(x)$. In the proof of Theorem 1.4, $b$ satisfies (1.7), and we proceed exactly as in Section 4.4.1 above, and then use 
(1.15). In the proof of Theorem 1.5, $b$ satisfies (1.17) and we proceed exactly as in Section 4.4 .2 above, and then use (1.18). In both cases we get

$$
\int_{0}^{T} \int_{\Omega^{2}}\left|I_{b}(x)\right||u(y)|^{2} d x d y d t \lesssim\|u(0)\|_{L^{2}}^{2}\left[\|u(0)\|_{\dot{H}^{\frac{1}{2}}}^{2}+\|u(T)\|_{\dot{H}^{\frac{1}{2}}}^{2}\right] .
$$

For the term containing $f(u)$ we write

$$
\begin{gathered}
\left.C_{a} \int_{0}^{T}\left|\int_{\Omega^{2}}\langle x\rangle^{-1-\delta}[f(u(x)) \overline{u(x)}-2 F(u(x))]\right| u(y)\right|^{2} d x d y d t \\
\lesssim\|u(0)\|_{L^{2}}\left[\|u(0)\|_{\dot{H}^{\frac{1}{2}}}^{2}+\|u(T)\|_{\dot{H}^{\frac{1}{2}}}^{2}\right]
\end{gathered}
$$

by (1.15) in the proof of Theorem 1.4 and (1.18) in the proof of Theorem 1.5, and this concludes the proof of (5.12).

5.4. The main term. Integrating in time the inequality $(5.5)$ on $[0, T]$ and collecting estimates $(5.11),(5.12)$ and the results of Section 5.1, we have proved that

$$
-\int_{0}^{T} \int_{\Omega^{2}} A_{x}^{2} \psi(x-y)|u(x)|^{2}|u(y)|^{2} d x d y d t \lesssim\|u(0)\|_{L^{2}}^{2}\left[\|u(0)\|_{\dot{H}^{\frac{1}{2}}}^{2}+\|u(T)\|_{\dot{H}^{\frac{1}{2}}}^{2}\right] .
$$

We now compute explicitly the quantity $A_{x}^{2} \psi$ : we have

$$
A_{x}^{2} \psi(x-y)=S(x, y)+E(x, y)
$$

where, using the notations

$$
\widetilde{a}=\widetilde{a}(x, y)=a(x)(\widehat{x-y}) \cdot(\widehat{x-y}), \quad \widehat{x}=\frac{x}{|x|},
$$

$S(x, y)$ and $E(x, y)$ are given by

$$
\begin{aligned}
S(x, y)= & \widetilde{a}^{2} \psi^{I V}(x-y)+\left[2 \bar{a}(x) \widetilde{a}-6 \widetilde{a}^{2}+4|a(x) \widehat{(x-y)}|^{2}\right] \frac{\psi^{\prime \prime \prime}(x-y)}{|x-y|}+ \\
& +\left[2 a_{\ell m}(x) a_{\ell m}(x)+\bar{a}^{2}(x)-6 \bar{a}(x) \widetilde{a}+15 \widetilde{a}^{2}-12|a(x)(\widehat{x-y})|^{2}\right] \times \\
& \times\left(\frac{\psi^{\prime \prime}(x-y)}{|x-y|^{2}}-\frac{\psi^{\prime}(x-y)}{|x-y|^{3}}\right)
\end{aligned}
$$

and

$$
\begin{aligned}
& E(x, y)=\widetilde{a} a_{\ell m ; \ell}(x)(\widehat{x-y})_{m} \psi^{\prime \prime \prime}(x-y)+(\bar{a}(x)-\widetilde{a}) a_{j k ; j}(x)(\widehat{x-y})_{k}\left(\frac{\psi^{\prime \prime}(x-y)}{|x-y|}-\frac{\psi^{\prime}(x-y)}{|x-y|^{2}}\right)+ \\
& \left.+\left[\partial_{j}\left(a_{j k}(x) a_{\ell m ; k}(x)(\widehat{x-y})_{\ell}(\widehat{x-y})_{m}\right)+\partial_{j}\left(a_{j k}(x) a_{\ell m}(x)\right) \partial_{k}(\widehat{(x-y})_{\ell}(\widehat{x-y})_{m}\right)\right] \times \\
& \times\left(\psi^{\prime \prime}(x-y)-\frac{\psi^{\prime}(x-y)}{|x-y|}\right) \\
& +\left(A_{x} \bar{a}(x)\right) \frac{\psi^{\prime}(x-y)}{|x-y|} \\
& +2 a_{j k}(x) a_{\ell m ; k}(x)(\widehat{x-y})_{\ell}(\widehat{x-y})_{m}(\widehat{x-y})_{j}\left(\psi^{\prime \prime \prime}(x-y)-\frac{\psi^{\prime \prime}(x-y)}{|x-y|}\right) \\
& +2 a(x)\left(\nabla \bar{a}(x), \nabla \frac{\psi^{\prime}(x-y)}{|x-y|}\right)+A_{x}\left(a_{\ell m ; \ell}(x)(\widehat{x-y})_{m} \psi^{\prime}(x-y)\right) .
\end{aligned}
$$

With long but elementary computations, for $n \geq 3$ and $\sigma \geq 0$ we have that

$|E(x, y)| \leq 5 n C_{a}\left(N+C_{a}\right)\left[\frac{1}{\langle x\rangle^{1+\delta}|x-y|\langle x-y\rangle_{\sigma}}+\frac{1}{\langle x\rangle^{1+\delta}|x|\langle x-y\rangle_{\sigma}}+\frac{1}{\langle x\rangle^{1+\delta}|x|^{2}}\right]$,

whence

$$
\int_{\Omega^{2}} E(x, y)|u(x)|^{2}|u(y)|^{2} d x d y \lesssim C_{a}[I+I I+I I I]
$$

with an implicit constant depending on $N$ and $n$, where

$$
I=\int_{\Omega^{2}} \frac{|u(x)|^{2}|u(y)|^{2}}{\langle x\rangle^{1+\delta}|x-y|^{2}} d x d y, \quad I I=\int_{\Omega^{2}} \frac{|u(x)|^{2}|u(y)|^{2}}{\langle x\rangle^{1+\delta}|x||x-y|} d x d y
$$


and

$$
I I I=\int_{\Omega^{2}} \frac{|u(x)|^{2}|u(y)|^{2}}{\langle x\rangle^{1+\delta}|x|^{2}} d x d y .
$$

We now extend $u(t, x)$ as zero outside $\Omega$, i.e. we define the function $U(t, x)$ as

$$
U(t, x)=u(t, x) \text { for } x \in \Omega, \quad U(t, x)=0 \text { for } x \notin \Omega .
$$

Before proceeding further, we need the following Lemma:

Lemma 5.3. Let $n \geq 3, \delta \in(0,1]$. There exist $\eta=\eta(n, \delta)>0$ such that for all $f \in \mathscr{S}$

$$
\begin{aligned}
\left\||D|^{\frac{3-n}{2}-1} \frac{f}{\langle\cdot\rangle^{1+\delta}}\right\|_{L^{2}\left(\mathbb{R}^{n}\right)} & \leq \eta\left\||D|^{\frac{3-n}{2}} f\right\|_{L^{2}\left(\mathbb{R}^{n}\right)}, \\
\left\||D|^{\frac{3-n}{2}-1} \frac{f}{|\cdot|^{\frac{1}{2}}\langle\cdot\rangle^{\frac{1}{2}+\delta}}\right\|_{L^{2}\left(\mathbb{R}^{n}\right)} & \leq \eta\left\||D|^{\frac{3-n}{2}} f\right\|_{L^{2}\left(\mathbb{R}^{n}\right)} .
\end{aligned}
$$

Proof. We prove the first inequality. By duality, it is equivalent to prove that

$$
\left\||D|^{\frac{n-3}{2}} \frac{f}{\langle x\rangle^{1+\delta}}\right\|_{L^{2}\left(\mathbb{R}^{n}\right)} \lesssim\left\||D|^{\frac{n-3}{2}+1} f\right\|_{L^{2}\left(\mathbb{R}^{n}\right)} .
$$

If $n=3,(5.16)$ is a simple consequence of Hardy inequality (2.11), in the case $y=0, b \equiv 0$. If $n \geq 4$, by the Kato-Ponce inequality (see e.g. [18]) and Sobolev embedding, we have

$$
\begin{aligned}
\||D|^{\frac{n-3}{2}} & \frac{f}{\langle x\rangle^{1+\delta}} \|_{L^{2}\left(\mathbb{R}^{n}\right)} \\
& \lesssim\left\||D|^{\frac{n-3}{2}} f\right\|_{L^{\frac{2 n}{n-2}}\left(\mathbb{R}^{n}\right)}\left\|\langle x\rangle^{-1-\delta}\right\|_{L^{n}}+\left\||D|^{\frac{n-3}{2}}\langle x\rangle^{-1}\right\|_{L^{\frac{2 n}{n-1}}}\|f\|_{L^{2 n}} \\
& \lesssim\left\||D|^{\frac{n-3}{2}+1} f\right\|_{L^{2}}
\end{aligned}
$$

where the implicit constants clearly depend only on $n$ and $\delta$. The proof of the second inequality is analogous.

Now, to estimate $I$ we write

$$
\begin{aligned}
I & =\int_{\mathbb{R}^{n}} \frac{|U(x)|^{2}}{\langle x\rangle^{1+\delta}} \int_{\mathbb{R}^{n}} \frac{|U(y)|^{2}}{|x-y|^{2}} d y d x \simeq \int_{\mathbb{R}^{n}} \frac{|U(x)|^{2}}{\langle x\rangle^{1+\delta}}|D|^{2-n}|U(x)|^{2} d x \\
& =\int_{\mathbb{R}^{n}}|D|^{\frac{3-n}{2}-1}\left(\langle x\rangle^{-1-\delta}|U(x)|^{2}\right)|D|^{\frac{3-n}{2}}|U(x)|^{2} d x \\
& \leq\left\||D|^{\frac{3-n}{2}-1} \frac{|U|^{2}}{\langle x\rangle^{1+\delta}}\right\|_{L^{2}}\left\||D|^{\frac{3-n}{2}}|U|^{2}\right\|_{L^{2}}
\end{aligned}
$$

and applying Lemma 5.3 we obtain

$$
I \leq C(n, \delta)\left\||D|^{\frac{3-n}{2}}|U|^{2}\right\|_{L^{2}}^{2} .
$$

Next we split the integral $I I$

$$
I I=\int_{\mathbb{R}^{2 n}} \frac{|U(x)|^{2}|U(y)|^{2}}{\langle x\rangle^{1+\delta}|x||x-y|} d x d y=\int_{A}+\int_{B}
$$


in the regions $A=\{(x, y): 2|x| \geq|y|\}$ and $B=\{(x, y): 2|x|<|y|\}$. On $A$ we have

$$
\begin{aligned}
\int_{A} \frac{|U(x)|^{2}|U(y)|^{2}}{\langle x\rangle^{1+\delta}|x||x-y|} d x d y & \lesssim \int_{A} \frac{|U(x)|^{2}|U(y)|^{2}}{\langle x\rangle^{\frac{1}{2}+\frac{\delta}{2}}|x|^{\frac{1}{2}}\langle y\rangle^{\frac{1}{2}+\frac{\delta}{2}}|y|^{\frac{1}{2}}|x-y|} d x d y \\
& \leq \int_{\mathbb{R}^{2 n}} \frac{|U(x)|^{2}}{\langle x\rangle^{\frac{1}{2}+\frac{\delta}{2}}|x|^{\frac{1}{2}}} \frac{1}{|x-y|} \frac{|U(y)|^{2}}{\langle y\rangle^{\frac{1}{2}+\frac{\delta}{2}}|y|^{\frac{1}{2}}} d x d y \\
& =\int_{\mathbb{R}^{n}} \frac{|U(x)|^{2}}{\langle x\rangle^{\frac{1}{2}+\frac{\delta}{2}}|x|^{\frac{1}{2}}}|D|^{1-n} \frac{|U(x)|^{2}}{\langle x\rangle^{\frac{1}{2}+\frac{\delta}{2}}|x|^{\frac{1}{2}}} d x \\
& =\left\||D|^{\frac{1-n}{2}} \frac{|U|^{2}}{|\cdot|^{\frac{1}{2}}\langle\cdot\rangle^{\frac{1}{2}+\frac{\delta}{2}}}\right\|_{L^{2}\left(\mathbb{R}^{n}\right)}^{2} \leq C(n, \delta)\left\||D|^{\frac{3-n}{2}}|U|^{2}\right\|_{L^{2}\left(\mathbb{R}^{n}\right)}^{2},
\end{aligned}
$$

where in the last step we used Lemma 5.3. On the region $B$ we have $|x| \lesssim|x-y|$, hence

$$
\int_{B} \frac{|U(x)|^{2}|U(y)|^{2}}{\langle x\rangle^{1+\delta}|x||x-y|} d x d y \lesssim \int_{B} \frac{|U(x)|^{2}|U(y)|^{2}}{\langle x\rangle^{1+\delta}|x|^{2}} d x d y \leq I I I
$$

Summing up, we have proved the estimate

$$
\begin{aligned}
-\int_{\Omega^{2}} A_{x}^{2} \psi(x-y)|u(x)|^{2}|u(y)|^{2} d x d y & \\
& \gtrsim-\int_{\Omega^{2}} S(x, y) d x d y-I I I-C(n, N, \delta) C_{a}\left\||D|^{\frac{3-n}{2}}|U|^{2}\right\|_{L^{2}\left(\mathbb{R}^{n}\right)}^{2}
\end{aligned}
$$

with an implicit constant depending on $N, n$ only.

5.4.1. Proof of Theorem 1.4. In this case, the expression for $S$ simplifies:

$S(x, y)=-|x-y|^{-3}\left[2 a_{l m}(x) a_{l m}(x)+\bar{a}^{2}(x)-6 \bar{a}(x) \widetilde{a}(x)+15 \widetilde{a}^{2}-12|a(x)(\widehat{x-y})|^{2}\right]$.

Now recalling (4.19), we see that if $N / \nu-1$ is small enough we have

$$
-S(x, y) \geq \epsilon_{0}|x-y|^{-3}
$$

for some strictly positive constant $\epsilon_{0}$. This implies

$$
\begin{aligned}
\int_{\Omega^{2}}-S(x, y)|u(x)|^{2}|u(y)|^{2} d x d y & \geq \epsilon_{0} \int_{\Omega^{2}} \frac{|u(x)|^{2}|u(y)|^{2}}{|x-y|^{3}} d x d y \\
& =\epsilon_{0}\left\||D|^{\frac{3-n}{2}}|U|^{2}\right\|_{L^{2}\left(\mathbb{R}^{n}\right)}^{2} .
\end{aligned}
$$

and, from (5.18), we get

$-\int_{\Omega^{2}} A_{x}^{2} \psi(x-y)|u(x)|^{2}|u(y)|^{2} d x d y \gtrsim-I I I+\left(\epsilon_{0}-C(n, N, \delta) C_{a}\right)\left\||D|^{\frac{3-n}{2}}|U|^{2}\right\|_{L^{2}\left(\mathbb{R}^{n}\right)}^{2}$ with an implicit constant depending on $N, n$ only. If $C_{a}$ is sufficiently small (with respect to $N, n, \nu$ and $\delta$ ), we obtain

$$
\gtrsim-I I I+|||D|^{\frac{3-n}{2}}|U|^{2} \|_{L^{2}\left(\mathbb{R}^{n}\right)}^{2}
$$

and integrating in time on $[0, T]$ and recalling (5.14), we arrive at the estimate

$$
\left\||D|^{\frac{3-n}{2}}|U|^{2}\right\|_{L_{T}^{2} L_{x}^{2}}^{2} \lesssim \int_{0}^{T} I I I d t+\|u(0)\|_{L^{2}}^{2}\left[\|u(0)\|_{\dot{H}^{\frac{1}{2}}}^{2}+\|u(T)\|_{\dot{H}^{\frac{1}{2}}}^{2}\right] .
$$

Note that by (2.7) we can write

$$
\int_{0}^{T} \operatorname{III} d t \leq\|u(0)\|_{L^{2}}^{2}\left\||x|^{-1}\langle x\rangle^{-\frac{1}{2}-\frac{\delta}{2}} u\right\|_{L_{x}^{2} L_{T}^{2}}^{2} \lesssim\|u(0)\|_{L^{2}}^{2}\|u\|_{\dot{X} L_{T}^{2}}^{2}
$$

and recalling (1.15) this gives

$$
\int_{0}^{T} I I I d t \leq\|u\|_{L_{x}^{2}}^{2}\|u\|_{L_{T}^{2} \dot{X}}^{2} \leq\|u(0)\|_{L^{2}}^{2}\left[\|u(0)\|_{\dot{H}^{\frac{1}{2}}}^{2}+\|u(T)\|_{\dot{H}^{\frac{1}{2}}}^{2}\right] .
$$


In conclusion we have

$$
\left\||D|^{\frac{3-n}{2}}|U|^{2}\right\|_{L_{T}^{2} L_{x}^{2}}^{2} \lesssim\|u(0)\|_{L^{2}}^{2}\left[\|u(0)\|_{\dot{H}^{\frac{1}{2}}}^{2}+\|u(T)\|_{\dot{H}^{\frac{1}{2}}}^{2}\right] .
$$

Note that

$$
\left\||D|^{\frac{3-n}{2}}|U|^{2}\right\|_{L_{x}^{2}}^{2}=\int_{\mathbb{R}^{n}}|D|^{\frac{3-n}{2}}|U|^{2} \cdot|D|^{\frac{3-n}{2}}|U|^{2} d x=\int_{\mathbb{R}^{n}}|U|^{2} \cdot|D|^{3-n}|U|^{2} d x
$$

and this can be written, apart from a constant,

$$
=\int_{\mathbb{R}^{2 n}} \frac{|U(x)|^{2}|U(y)|^{2}}{|x-y|^{3}} d x d y=\int_{\Omega^{2}} \frac{|u(x)|^{2}|u(y)|^{2}}{|x-y|^{3}} d x d y
$$

which concludes the proof of the Theorem.

5.4.2. Proof of Theorem 1.5. We recall the following identities for $a$ :

$$
\begin{gathered}
a=I+q, \quad a_{l m}=\delta_{l m}+q_{l m}, \\
\bar{a}=3+\bar{q}, \quad a_{l m} a_{l m}=3+2 \bar{q}+q_{l m} q_{l m}, \\
\widetilde{a}=1+\widetilde{q}, \quad|a(\widehat{x-y})|^{2}=1+2 \widetilde{q}+|q(\widehat{x-y})|^{2} .
\end{gathered}
$$

Starting from (5.15) and using formulas (5.4) and the previous identities, we obtain

$$
\begin{aligned}
-S(x, y) \geq & 15 \frac{\sigma^{4}}{\langle x-y\rangle_{\sigma}^{7}}+30 \widetilde{q} \frac{\sigma^{4}}{\langle x-y\rangle_{\sigma}^{7}}+(2 \bar{q}-6 \widetilde{q}+2 \bar{q} \widetilde{q}) \frac{3 \sigma^{2}}{\langle x-y\rangle_{\sigma}^{5}} \\
& +\left(4 \bar{q}-12 \widetilde{q}-6 \bar{q} \widetilde{q}-3 \widetilde{q}^{2}-12|q(\widehat{x-y})|^{2}\right) \frac{1}{\langle x-y\rangle_{\sigma}^{3}} .
\end{aligned}
$$

Since we have by assumption

$$
|\bar{q}| \leq 3 C_{I}\langle x\rangle^{-\delta}, \quad|\widetilde{q}| \leq C_{I}\langle x\rangle^{-\delta}, \quad|\widehat{q(x-y)}| \leq C_{I}\langle x\rangle^{-\delta} .
$$

this implies

$$
-S(x, y) \geq 15 \sigma^{4}\langle x-y\rangle_{\sigma}^{-7}-46 C_{I}\langle x\rangle^{-\delta}\langle x-y\rangle_{\sigma}^{-3} .
$$

From (5.18) and (5.20) we have

$$
\begin{aligned}
-\int_{\Omega^{2}} & A_{x}^{2} \psi(x-y)|u(x)|^{2}|u(y)|^{2} d x d y \\
\gtrsim & \int_{\Omega^{2}}\left(15 \frac{\sigma^{4}}{\langle x-y\rangle_{\sigma}^{7}}-\frac{46 C_{I}}{\langle x-y\rangle_{\sigma}^{3}}\right)|u(x)|^{2}|u(y)|^{2} d x d y \\
& \quad-I I I-C(n, N, \delta) C_{a}\|u\|_{L^{4}}^{4}
\end{aligned}
$$

with an implicit constant depending on $N, n$ only. We let $\sigma \rightarrow 0$ and integrate in $t$ on $[0, T]$ : recalling $(5.14)$, we get

$$
\left(15-46 C_{I}-C(n, N, \delta) C_{a}\right)\|u\|_{L_{T}^{4} L^{4}}^{4} \lesssim\|u(0)\|_{L^{2}}^{2}\left[\|u(0)\|_{\dot{H}^{\frac{1}{2}}}^{2}+\|u(T)\|_{\dot{H}^{\frac{1}{2}}}^{2}\right]+\int_{0}^{T} I I I d t .
$$

Note that by (4.32), (2.4), and (1.18) we have

$$
\begin{aligned}
\int_{0}^{T} I I I d t & \leq\|u(0)\|_{L^{2}}^{2}\left\||x|^{-1}\langle x\rangle^{-(1+\delta) / 2} u\right\|_{L_{x}^{2} L_{T}^{2}}^{2} \\
& \leq\|u(0)\|_{L^{2}}^{2} \delta^{-1}\left[\|u\|_{X L_{T}^{2}}^{2}+\left\|\nabla^{b} u\right\|_{Y L_{T}^{2}}^{2}\right] \\
& \lesssim\|u(0)\|_{L^{2}}^{2}\left[\|u(0)\|_{\dot{H}^{\frac{1}{2}}}^{2}+\|u(T)\|_{\dot{H}^{\frac{1}{2}}}^{2}\right] .
\end{aligned}
$$

If $C_{I}$ and $C_{a}$ are small enough, we get immediately the claim from (5.21) and (5.22). 


\section{Gaussian bounds And applications}

Let $L$ be the operator (1.2), (1.3) defined on an open set $\Omega \subseteq \mathbb{R}^{n}$. For the results in this section it is not necessary to assume any condition on $\Omega$ which may be an arbitrary open set; we shall anyway assume $\partial \Omega \in C^{1}$ for the sake of simplicity. First of all, we chack that $L$ can be realized as a selfadjoint operator, with Dirichlet b.c., under very weak assumptions on the coefficients:

Proposition 6.1. Let $n \geq 3$ and $\Omega \subseteq \mathbb{R}^{n}$ an open set with $C^{1}$ boundary. Consider the operator $L$ defined on $C_{c}^{\infty}(\Omega)$ by (1.2), (1.3), under the assumptions

$$
a \in L^{\infty}, \quad b \in L^{n, \infty}, \quad c \in L^{\frac{n}{2}, \infty}, \quad\left\|c_{-}\right\|_{L^{\frac{n}{2}, \infty}}<\epsilon .
$$

Then, if $\epsilon$ sufficiently small, $-L$ extends to a selfadjoint nonnegative operator in the sense of forms, and $D(-L)=H_{0}^{1}(\Omega) \cap H^{2}(\Omega)$ is a form core. Moreover we have

$$
(-L v, v)_{L^{2}}=\left\|(-L)^{\frac{1}{2}} v\right\|_{L^{2}}^{2} \simeq\|\nabla v\|_{L^{2}}^{2}, \quad\left\|(-L)^{\frac{1}{4}} v\right\|_{L^{2}} \simeq\|v\|_{\dot{H}^{\frac{1}{2}}} .
$$

Proof. We sketch the proof which is mostly standard, apart from the use of Lorentz spaces. The form

$$
q(v)=(-L v, v)_{L^{2}}=\int_{\Omega} a\left(\nabla^{b} v, \nabla^{b} v\right) d x+\int_{\Omega} c|v|^{2} d x
$$

is bounded on $H_{0}^{1}(\Omega)$ : indeed, by Hölder and Sobolev inequalities in Lorentz spaces,

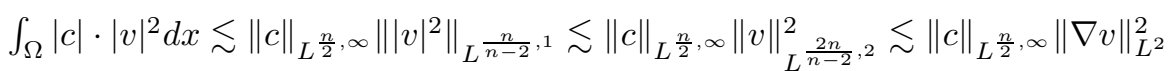

while by (2.15) we have $\left\|\nabla^{b} v\right\|_{L^{2}} \simeq\|\nabla v\|_{L^{2}}$. Thus if $\epsilon$ is sufficiently small we have $q(v) \simeq\|\nabla v\|_{L^{2}}$; in particular $q(v)$ is a symmetric, closed, nonnegative form on $H_{0}^{1}(\Omega)$, and defines a selfadjoint operator with $D(-L)=H^{2}(\Omega) \cap H_{0}^{1}(\Omega)$ which is also a core for $q$. The last property in (6.2) follows by complex interpolation, since $D\left((-L)^{s}\right)$ for $0 \leq s \leq 1$ is an interpolation family.

Under slightly stronger assumptions, we can see that the heat flow $e^{t L}$ satisfies an upper gaussian bound; this will be a crucial tool in the following. Compare with [13] and [12] for similar results in the case $a=I, \Omega=\mathbb{R}^{n}$. Note that for $a, b, c \in L^{\infty}$ with $c \geq 0$ the bound is proved in Corollary 6.14 of [26]. The following result is sufficient for our purposes, although the assumptions on the coefficients could be further relaxed.

Proposition 6.2. Let $n \geq 3$. Assume the operator $L$ is defined as in (1.2), (1.3) on the open set $\Omega \subseteq \mathbb{R}^{n}$ with $C^{1}$ boundary, and that $a, b, c$ satisfy

$$
a \in L^{\infty}, \quad b \in L_{l o c}^{4} \cap L^{n, \infty}, \quad \nabla \cdot b \in L_{l o c}^{2}, \quad c \in L^{\frac{n}{2}, 1}, \quad\left\|c_{-}\right\|_{L^{\frac{n}{2}, 1}}<\epsilon .
$$

Then, if $\epsilon$ is sufficiently small, the heat kernel $e^{t L}$ satisfies, for some $C, C^{\prime}>0$,

$$
\left|e^{t L}(x, y)\right| \leq C^{\prime} t^{-\frac{n}{2}} e^{-\frac{|x-y|^{2}}{C t}}, \quad t>0, x, y \in \Omega .
$$

Proof. We can apply Proposition 6.1 since the assumptions are stronger. When $b=c=0$, the gaussian bound follows directly from Corollary 6.14 in [27]; note that in this case the kernel of $e^{t L}$ is $\geq 0$.

Next, in order to handle the case $b \neq 0, c=0$, we adapt the proof of Lemma 10 in [21]. Let $\phi \in C_{c}^{\infty}(\Omega)$ and write $\phi_{\delta}=\sqrt{|\phi|^{2}+\delta^{2}}$ for $\delta>0$. It is easy to prove the pointwise inequality (recall notations $(2.1)$ )

$$
A \phi_{\delta} \geq \Re\left(\frac{\bar{\phi}}{\phi_{\delta}} A^{b} \phi\right)
$$

which implies, for all $\lambda>0$,

$$
(-A+\lambda) \phi_{\delta} \leq \Re\left(\frac{\bar{\phi}}{\phi_{\delta}}\left(-A^{b}+\lambda\right) \phi\right)+\lambda\left(\phi_{\delta}-\frac{|\phi|^{2}}{\phi_{\delta}}\right) .
$$


Proceeding as in [21], we obtain

$$
\left|\left(-A^{b}+\lambda\right)^{-1} f\right| \leq(-A+\lambda)^{-1}|f|
$$

and iterating we have for all $k \geq 0$

$$
\left|\left(-A^{b}+\lambda\right)^{-k} f\right| \leq(-A+\lambda)^{-k}|f|
$$

since $(-A+\lambda)^{-1}$ is positivity preserving (see Remark 1 in [21]). Then we deduce

$$
\left|e^{t A^{b}} \phi\right| \leq e^{t A}|\phi|
$$

via $e^{t A^{b}}=\lim _{k \rightarrow \infty}\left(I-t A^{b} / n\right)^{-n}$, and applying the last formula to a delta sequence $\phi=\phi_{j}$ made of nonnegative functions, we conclude that the gaussian bound (6.4) is valid for $e^{t A^{b}}$.

It remains to consider the case $c \neq 0$. To this end we apply the theory of [22]. Let $U(t, s)$ be the propagator defined as $U(t, s) f=e^{(t-s) A^{b}} f$, for $t \geq s \geq 0$. By the gaussian bound just proved we have that $U(t, s)$ extends to a uniformly bounded operator $L^{1} \rightarrow L^{1}$ and $L^{\infty} \rightarrow L^{\infty}$, moreover $\|U(t, s)\|_{L^{1} \rightarrow L^{\infty}} \lesssim|t-s|^{-\frac{n}{2}}$; finally, the adjoint propagator $U_{*}(t, s):=(U(s, t))^{*}$ for $s \geq t \geq 0$ coincides with $U(s, t)$ since $A^{b}$ is selfadjoint, so that $U_{*}$ is strongly continuous on $L^{1}$ (notice that this last assumption is not actually necessary, as mentioned in the paper). Then by applying Theorem 3.10 from [22] we conclude that the gaussian bound, with possibly different constants, is satisfied also by the perturbed propagator $U_{c}=e^{t\left(A^{b}-c\right)}$, provided the potential $c$ is a Miyadera perturbation of both $U$ and $U_{*}$ such that $c_{-}$is Miyadera small with constants $(\infty, \gamma), \gamma<1$. The verification of this last condition, in the special case considered here, reduces to the following inequality, for all $s \geq 0$

$$
I:=\int_{s}^{+\infty}\left\|c(x) e^{(t-s) A^{b}} f\right\|_{L^{1}} d t \leq \gamma\|f\|_{L 1}
$$

(we are using formula (2.5) in [22] with the choices $\alpha=\infty, J=\mathbb{R}^{+}$and $p=1$ ) and the same inequality with $\gamma<1$ for $c_{-}$. The gaussian bound already proved for $e^{t A^{b}}$ implies

$$
I \lesssim \int_{\Omega} \int_{\Omega}|c(x)||f(y)| \int_{0}^{+\infty} t^{-\frac{n}{2}} e^{-\frac{|x-y|^{2}}{C t}} d t d y d x \lesssim\|f\|_{L^{1}} \sup _{y \in \Omega} \int_{\Omega} \frac{|c(x)|}{|x-y|^{n-2}} d x
$$

and by the Young inequality in Lorentz spaces we get

$$
I \lesssim\|c\|_{L^{\frac{n}{2}, 1}}\|f\|_{L^{1}}
$$

which concludes the proof (compare with the proof of Lemma 5.1 in [34]).

Proposition 6.3. Let $n \geq 3$. Assume the operator $L$ is defined as in (1.2), (1.3) on the open set $\Omega \subseteq \mathbb{R}^{n}$ with $C^{1}$ boundary, and that $a, b, c$ satisfy

$b^{2}+|\nabla \cdot b| \in L_{l o c}^{2}, \quad c \in L^{\frac{n}{2}, 1}, \quad\|a-I\|_{L^{\infty}}+\left\||b|+\left|a^{\prime}\right|\right\|_{L^{n, \infty}}+\left\|b^{\prime}\right\|_{L^{\frac{n}{2}, \infty}}+\left\|c_{-}\right\|_{L^{\frac{n}{2}, 1}}<\epsilon$.

If $\epsilon$ sufficiently small then for all $0 \leq \sigma \leq 1$ we have

$$
\left\|(-L)^{\sigma} v\right\|_{L^{p}} \simeq\left\|(-\Delta)^{\sigma} v\right\|_{L^{p}}, \quad 1<p<\frac{n}{2 \sigma} .
$$

Proof. The assumptions of the two previous Propositions are satisfied, thus $-L$ is selfadjoint, nonnegative, and the gaussian bound (6.4) is valid.

Consider first the case $\sigma=1$. Write the operator $L$ in the form

$$
L v=\sum_{j k} a_{j k} \partial_{j} \partial_{k} v+\sum_{j} \beta_{j} \partial_{j} v+\gamma_{0} v-c_{+} v
$$

where

$$
\beta_{k}=\sum_{j}\left(\partial_{j} a_{j k}+2 i a_{j k} b_{k}\right), \quad \gamma_{0}=\sum_{j, k} i \partial_{j}\left(a_{j k} b_{k}\right)-a(b, b)+c_{-} .
$$

Then by Hölder and Sobolev inequalities in Lorentz spaces we have for $1<p<\frac{n}{2}$ $\|L v\|_{L^{p}} \leq\|a\|_{L^{\infty}}\left\|D^{2} v\right\|_{L^{p}}+\|\beta\|_{L^{n, \infty}}\|D v\|_{L^{\frac{n p}{n-p}, p}}+\left\|\gamma_{0}-c_{+}\right\|_{L^{\frac{n}{2}, \infty}}\|v\|_{L^{\frac{n p}{n-2 p}, p}} \lesssim\|\Delta v\|_{L^{p}}$. 
To prove the converse inequality, we first represent the operator $\left(-\Delta+c_{+}\right)^{-1}$ in the form

$$
\left(-\Delta+c_{+}\right)^{-1}=c(n) \int_{0}^{+\infty} e^{t\left(\Delta-c_{+}\right)} d t
$$

and we apply the gaussian bound to obtain

$$
\left|\left(-\Delta+c_{+}\right)^{-1}\right| \lesssim \int_{0}^{+\infty} e^{-\frac{|x-y|^{2}}{C t}} t^{-\frac{n}{2}} d t \lesssim|x-y|^{2-n} .
$$

As a consequence, using the Hardy-Sobolev inequality we get

$$
\left\|\left(-\Delta+c_{+}\right)^{-1} v\right\|_{L^{\frac{n p}{n-2 p}}} \lesssim\|v\|_{L^{p}} \quad \text { i.e. } \quad\|v\|_{L^{\frac{n p}{n-2 p}}} \lesssim\left\|\left(-\Delta+c_{+}\right) v\right\|_{L^{p}}
$$

for all

$$
1<p<\frac{n}{2}
$$

In particular this gives (since $\left\|c_{+}\right\|_{L^{\frac{n}{2}, \infty}} \lesssim\left\|c_{+}\right\|_{L^{\frac{n}{2}, 1}}$ )

$$
\|\Delta v\|_{L^{p}} \leq\left\|\left(\Delta-c_{+}\right) v\right\|_{L^{p}}+\left\|c_{+}\right\|_{L^{\frac{n}{2}, \infty}}\|v\|_{L^{\frac{n p}{n-2 p}}} \lesssim\left\|\left(\Delta-c_{+}\right) v\right\|_{L^{p}}, \quad 1<p<\frac{n}{2} .
$$

Adding and subtracting the remaining terms in $L$ in the last term, we obtain

$$
\left\|\left(\Delta-c_{+}\right) v\right\|_{L^{p}} \leq\|L v\|_{L^{p}}+\left\|\sum\left(a_{j k}-\delta_{j k}\right) \partial_{j} \partial_{k} v\right\|_{L^{p}}+\left\|\sum \beta_{k} \partial_{k} v\right\|_{L^{p}}+\left\|\gamma_{0} v\right\|_{L^{p}}
$$

and a last application of Hölder and Sobolev inequalities gives

$$
\|\Delta v\|_{L^{p}} \lesssim\left\|\left(\Delta-c_{+}\right) v\right\|_{L^{p}} \lesssim\|L v\|_{L^{p}}+\epsilon\|\Delta v\|_{L^{p}} .
$$

If $\epsilon$ is sufficiently small we can subtract the last term from the left hand side, and the proof of the case $\sigma=1$ is concluded. The case $\sigma=0$ is trivial, and the remaining cases will be handled by Stein-Weiss complex interpolation.

Indeed, consider the family of operators $T_{z}=(-L)^{z}(-\Delta)^{-z}$ for $0 \leq \Re z \leq 1$; our first goal is to prove that $T_{z}: L^{p} \rightarrow L^{p}$ is bounded provided $1<p<n /(2 \Re z)$, which implies the inequality $\lesssim$ in (6.8). Note that the following arguments work with trivial modifications also for $-1 \leq \Re z \leq 0$ and give then the converse inequality $\gtrsim$.

$T_{z}$ is obviously an analytic family of operators, and $T^{i y}$ for real $y$ is bounded on all $L^{p}$ with $1<p<\infty$, with a norm growing at most polynomially as $|y| \rightarrow \infty$. This property is well known for $(-\Delta)^{i y}$, while for $L^{i y}$ it follows from the theory developed in [14] (see also [4] for the case $\Omega=\mathbb{R}^{n}$ ), which requires the sole assumption that $L$ satisfies a gaussian bound like (6.4). A standard application of the Stein-Weiss theorem then gives the claim.

To conclude this section we construct a family of regularizing operators which will be needed later in the proof of $H^{1}$ well posedness; what follows is an adaptation of Section 1.5 in [7]. Assume that $\Omega$ and $L$ satisfy the assumptions of the previous Proposition. We define for $0<\epsilon \leq 1$ the operators

$$
J_{\epsilon}:=(I-\epsilon L)^{-1} \equiv \epsilon^{-1} R\left(-\epsilon^{-1}\right)
$$

where $R(z)=(-L-z)^{-1}$ is the resolvent operator of $-L$. Then for every $f \in$ $H^{-1}(\Omega)$ the function $u=J_{\epsilon} f \in H_{0}^{1}(\Omega)$ is well defined as the unique weak solution of the elliptic equation

$$
-L u+\epsilon^{-1} u=\epsilon^{-1} f \text {. }
$$

Thus $J_{\epsilon}: H^{-1}(\Omega) \rightarrow H_{0}^{1}(\Omega)$ is a bounded operator, $L: H_{0}^{1}(\Omega) \rightarrow H^{-1}(\Omega)$ is bounded, we have the equivalence $\|(I-L) v\|_{H^{-1}(\Omega)} \simeq\|v\|_{H_{0}^{1}(\Omega)}$ and the estimates

$$
\left\|J_{\epsilon} v\right\|_{H_{0}^{1}(\Omega)} \leq C \epsilon^{-1}\|v\|_{H^{-1}(\Omega)}, \quad\left\|J_{\epsilon} v\right\|_{H^{2}(\Omega)} \leq C \epsilon^{-1}\|v\|_{L^{2}(\Omega)}
$$

by standard elliptic theory, with a $C$ independent of $\epsilon$. Further we have

$$
\left\|J_{\epsilon} v\right\|_{H_{0}^{1}(\Omega)} \leq C\|v\|_{H_{0}^{1}(\Omega)}, \quad\left\|J_{\epsilon} v\right\|_{L^{2}(\Omega)} \leq C\|v\|_{L^{2}(\Omega)}, \quad\left\|J_{\epsilon} v\right\|_{H^{-1}(\Omega)} \leq C\|v\|_{H^{-1}(\Omega)}
$$

and by complex interpolation

$$
\left\|J_{\epsilon} v\right\|_{H_{0}^{1}(\Omega)} \leq C \epsilon^{-\frac{1}{2}}\|v\|_{L^{2}(\Omega)}, \quad\left\|J_{\epsilon} v\right\|_{L^{2}(\Omega)} \leq C \epsilon^{-\frac{1}{2}}\|v\|_{H^{-1}(\Omega)} .
$$


Then, using the identity $J_{\epsilon}-I=J_{\epsilon}(I-I+\epsilon L)=\epsilon J_{\epsilon} L$, we deduce

$$
\left\|\left(J_{\epsilon}-I\right) v\right\|_{H^{-1}(\Omega)} \leq C \epsilon\|L v\|_{H^{-1}(\Omega)} \leq C^{\prime} \epsilon\|v\|_{H_{0}^{1}(\Omega)} .
$$

Note that if $v \in H^{-1}(\Omega)$ only, we can still approximate it with $\phi \in C_{c}^{\infty}(\Omega)$ to get

$$
\left\|\left(J_{\epsilon}-I\right) v\right\|_{H^{-1}(\Omega)} \leq C\|v-\phi\|_{H^{-1}(\Omega)}+C \epsilon\|\phi\|_{H_{0}^{1}(\Omega)}
$$

and this implies

$$
\forall v \in H^{-1}(\Omega) \quad J_{\epsilon} v \rightarrow v \text { in } H^{-1}(\Omega) \text { as } \epsilon \rightarrow 0 .
$$

We also obtain

$$
\left\|\left(J_{\epsilon}-I\right) v\right\|_{L^{2}(\Omega)} \leq C\left\|\left(J_{\epsilon}-I\right) v\right\|_{H_{0}^{1}(\Omega)}^{\frac{1}{2}}\left\|\left(J_{\epsilon}-I\right) v\right\|_{H^{-1}(\Omega)}^{\frac{1}{2}} \leq C^{\prime} \epsilon^{\frac{1}{2}}\|v\|_{H_{0}^{1}(\Omega)}
$$

and an argument similar to the previous one gives

$$
\forall v \in L^{2}(\Omega) \quad J_{\epsilon} v \rightarrow v \text { in } L^{2}(\Omega) \text { as } \epsilon \rightarrow 0 .
$$

Finally, by the equivalence $\left\|\left(J_{\epsilon}-I\right) v\right\|_{H_{0}^{1}(\Omega)} \simeq\left\|\left(J_{\epsilon}-I\right)(I-L) v\right\|_{H^{-1}(\Omega)}$ we get

$$
\forall v \in H_{0}^{1}(\Omega) \quad J_{\epsilon} v \rightarrow v \text { in } H_{0}^{1}(\Omega) \text { as } \epsilon \rightarrow 0 .
$$

Concerning the convergence in $L^{p}(\Omega)$ we have:

Proposition 6.4. Let $p \in[1, \infty)$ and let $\Omega$ and $L$ satisfy the assumptions of Proposition 6.3. Then $J_{\epsilon}$ extends to a bounded operator on $L^{p}(\Omega)$ and the following estimate holds for $0<\epsilon \leq 1$

$$
\left\|J_{\epsilon} v\right\|_{L^{p}(\Omega)} \leq C\|v\|_{L^{p}(\Omega)}
$$

with a constant depending on $p$ but not of $\epsilon$. Moreover, for $1<p<\infty$ we have

$$
\forall v \in L^{p}(\Omega) \quad J_{\epsilon} v \rightarrow v \text { in } L^{p}(\Omega) \text { as } \epsilon \rightarrow 0 .
$$

Proof. Let $\phi:(0, \infty) \rightarrow[0, \infty)$ be a smooth nondecreasing function with $\phi(s), s \phi^{\prime}(s)$ bounded. Starting from the identity

$\Re(-L v \cdot \phi(|v|) \bar{v})+\nabla \cdot\left\{\Re\left(\bar{v} \phi(|v|) a \nabla^{b} v\right)\right\}=\phi(|v|) a\left(\nabla^{b} v, \nabla^{b} v\right)+\frac{\phi^{\prime}(|v|)}{|v|}\left|\Re\left(\bar{v} \cdot a \nabla^{b} v\right)\right|^{2}+c \phi(|v|)|v|^{2}$, and proceeding exactly as in the proof of Proposition 1.5.1 in [7], we obtain (6.17). In order to prove (6.18), we can assume $v \in C_{c}^{\infty}(\Omega)$ (as above). Then by the interpolation inequality in $L^{p}$ we can write for all $0<\theta<1$

$$
\left\|\left(J_{\epsilon}-I\right) v\right\|_{L^{\frac{2}{1-\theta}}} \leq\left\|\left(J_{\epsilon}-I\right) v\right\|_{L^{1}}^{\theta}\left\|\left(J_{\epsilon}-I\right) v\right\|_{L^{2}}^{1-\theta} \leq C\|v\|_{L^{1}}^{\theta} \cdot\left\|\left(J_{\epsilon}-I\right) v\right\|_{L^{2}}^{\theta}
$$

where we used (6.17), and by (6.15) we conclude that $J_{\epsilon} v \rightarrow v$ in $L^{p}(\Omega)$ for all $p=\frac{2}{1-\theta} \in(1,2)$. A similar argument gives the result for $p \in(2, \infty)$, and the case $p=2$ we already know.

\section{Global existence and Scattering: Proof of Theorem 1.7}

Throughout this section $\Omega \subseteq \mathbb{R}^{n}$ is an open set with $C^{1}$ boundary, $n \geq 3$, while $L$ is the unbounded operator on $L^{2}(\Omega)$ with Dirichlet boundary conditions under the assumptions of Proposition 6.1. As explained in the Introduction, we shall work under the black box Assumption (S) which ensures that the necessary Strichartz estimates are available. Notice that we are restricting the range of admissible indices at the left hand side for the derivative of the flow $\nabla e^{i t L}$.

Our goal is to extend the usual local and global $H^{1}$ theory to the NLS with variable coefficients

$$
i u_{t}-L u+f(u)=0, \quad u(0, x)=u_{0}(x) .
$$


We shall sketch only the essential results which will be needed in the proof of scattering, and not aim at the greatest possible generality. In the following we use the notations

$$
L_{T}^{p} L^{q}=L^{p}\left(0, T ; L^{q}(\Omega)\right), \quad C_{T} H_{0}^{1}=C\left([0, T], H_{0}^{1}(\Omega)\right) .
$$

Proposition 7.1 (Local existence in $H_{0}^{1}(\Omega)$ ). Let $n \geq 3$ and assume $(S)$ holds, while $f \in C^{1}(\mathbb{C}, \mathbb{C})$ satisfies

$$
|f(z)| \lesssim|z|^{\gamma}, \quad|f(z)-f(w)| \lesssim(|z|+|w|)^{\gamma-1}|z-w| \text { for some } 1 \leq \gamma<1+\frac{4}{n-2} \text {. }
$$

Then for all $u_{0} \in H_{0}^{1}(\Omega)$ there exists $T=T\left(\left\|u_{0}\right\|_{H^{1}}\right)$ and a unique solution $u \in$ $C\left([0, T] ; H_{0}^{1}(\Omega)\right)$.

Proof. The proof is standard; we sketch the main steps in order to check that the restriction $q_{1}<n$ imposed in $(\mathrm{S})$ is harmless. We apply a fixed point argument to the map $\Phi: v \mapsto u$ defined as the solution of $i u_{t}-L u+f(v)=0, u(0, x)=$ $u_{0}$, working in a suitable bounded subset of the space $X_{T}=C\left([0, T] ; H_{0}^{1}(\Omega)\right) \cap$ $L^{p}\left(0, T ; W^{1, q}(\Omega)\right)$ for an appropriate choice of $(p, q)$, endowed with the distance $d(u, v)=\|u-v\|_{C_{T} L^{2} \cap L_{T}^{p} L^{q}}$; note that bounded subsets of $X_{T}$ are complete with this distance.

In order to choose the indices we pick a real number $k$ such that

$$
n<2 k n<n+2, \quad \gamma(n-4)+2<2 k n<\gamma(n-2)+2 .
$$

Note that for all $n \geq 3$ and all $1<\gamma<\frac{n+2}{n-2}$ the two intervals in (7.3) have a nonempty intersection. Moreover, the couples $\left(p_{j}, q_{j}\right)$ defined by

$$
p_{1}=\frac{4 \gamma}{2+\gamma(n-2)-2 k n}, \quad q_{1}=\frac{\gamma n}{k n+\gamma-1}, \quad p_{2}=\frac{4}{2 k n-n}, \quad q_{2}=\frac{1}{1-k}
$$

are admissible and we can use the estimates in $(\mathrm{S})$, provided $q_{1}<n$ which will be checked at the end. We choose then $(p, q)=\left(p_{1}, q_{1}\right)$ in the definition of $X_{T}$. Applying Strichartz estimates on a time interval $[0, T]$ with $T$ to be chosen, we have for $u=\Phi(v)$

$$
\|\nabla u\|_{L_{T}^{p_{1}} L^{q_{1}}}+\|\nabla u\|_{L_{T}^{\infty} L^{2}} \lesssim\left\|u_{0}\right\|_{\dot{H}^{1}}+\|\nabla f(v)\|_{L_{T}^{p_{2}^{\prime}} L^{q_{2}^{\prime}}} .
$$

By Hölder and Sobolev inequalities, using the assumptions on $f$, we have

$$
\|\nabla f(v)\|_{L_{T}^{p_{2}^{\prime} L_{2}^{q_{2}^{\prime}}}} \lesssim\|\| v\left\|_{L_{\frac{\gamma n}{k n-1}}^{\gamma-1}}\right\| \nabla v\left\|_{L^{q_{1}}}\right\|_{L_{T}^{p_{2}^{\prime}}} \lesssim\|\nabla v\|_{L_{T}^{\gamma p_{2}^{\prime} L^{q_{1}}}}^{\gamma} .
$$

Now we note that the condition $\gamma<\frac{n+2}{n-2}$ is equivalent to $\gamma p_{2}^{\prime}<p_{1}$, thus Hölder inequality on $[0, T]$ gives

$$
\|\nabla u\|_{L_{T}^{p_{1}} L^{q_{1}}}+\|\nabla u\|_{L_{T}^{\infty} L^{2}} \lesssim\left\|u_{0}\right\|_{\dot{H}^{1}}+T^{\frac{1}{p_{2}^{\prime}}-\frac{\gamma}{p_{1}}}\|\nabla v\|_{L_{T}^{p_{1}} L^{q_{1}}}^{\gamma}
$$

with a strictly positive power of $T$. An analogous computation gives

$$
\|u\|_{L_{T}^{p_{1}} L^{q_{1}}}+\|u\|_{L_{T}^{\infty} L^{2}} \lesssim\left\|u_{0}\right\|_{L^{2}}+T^{\frac{1}{p_{2}^{\prime}}-\frac{\gamma}{p_{1}}}\|\nabla v\|_{L_{T}^{p_{1}} L^{q_{1}}}^{\gamma-1}\|v\|_{L_{T}^{p_{1}} L^{q_{1}}}
$$

and summing up we have proved

$$
\|\Phi(v)\|_{X_{T}} \lesssim\left\|u_{0}\right\|_{H^{1}}+T^{\sigma}\|v\|_{X_{T}}^{\gamma}, \quad \sigma=\frac{1}{p_{2}^{\prime}}-\frac{\gamma}{p_{1}}>0 .
$$

Similar computations give

$$
d\left(\Phi\left(v_{1}\right), \Phi\left(v_{2}\right)\right) \lesssim T^{\sigma}\left(1+\left\|v_{1}\right\|_{X_{T}}+\left\|v_{2}\right\|_{X_{T}}\right)^{\gamma-1}\left\|v_{1}-v_{2}\right\|_{L_{T}^{p_{1}} L^{q_{1}}}
$$

and by a standard contraction argument on a suitable ball of $X_{T}$ we obtain the existence of a fixed point i.e. a solution of (7.1) provided $T$ is smaller than a quantity $T\left(\left\|u_{0}\right\|_{H^{1}}\right)$ which depends only on the $H^{1}$ norm of the initial data. 
It remains to check the claim $q_{1}<n$. Since $2 k n>n$ and $\gamma<\frac{n+2}{n-2}$ we have

$$
q_{1}=\frac{2 \gamma n}{2 k n+2 \gamma-2}<\frac{2 \gamma n}{n+2 \gamma-2}<\frac{2 n(n+2)}{n^{2}-2 n+8}
$$

and the last fraction is $\leq 3$ for all integers $n \geq 5$, while it is equal to $70 / 33<4$ for $n=4$ and to $30 / 11<3$ when $n=3$.

To prove uniqueness, if $u, v$ are two solutions in $C_{T} H^{1}$ for some $T>0$, we can write

$$
\|u-v\|_{L_{T}^{p} L^{\gamma+1}} \lesssim\|f(u)-f(v)\|_{L_{T}^{p^{\prime}} L^{(\gamma+1)^{\prime}}} \lesssim\|u-v\|_{L_{T}^{b} L^{\gamma+1}}\||u|+|v|\|_{L_{T}^{p_{0}} L^{\gamma+1}}^{\gamma-1}
$$

where

$$
p=\frac{4}{n} \frac{\gamma+1}{\gamma-1}, \quad \frac{1}{p_{0}}=\frac{1}{p}-\frac{1}{2}, \quad \frac{1}{b}=\frac{\gamma}{2}-\frac{\gamma}{p}+\frac{1}{2} .
$$

(note that we are not using Strichartz estimates of $\nabla u$ ), hence by Sobolev embedding

$$
\|u-v\|_{L_{T}^{p} L^{\gamma+1}} \lesssim\left(\|u\|_{L_{T}^{p_{0} H^{1}}}+\|v\|_{L_{T}^{p_{0} H^{1}}}\right)^{\gamma-1}\|u-v\|_{L_{T}^{b} L^{\gamma+1}}
$$

It is easy to check that $b<p$, thus we get

$$
\lesssim T^{\epsilon}\left(\|u\|_{L_{T}^{\infty} H^{1}}+\|v\|_{L_{T}^{\infty} H^{1}}\right)\|u-v\|_{L_{T}^{p} L^{\gamma+1}}
$$

for some $\epsilon>0$ and this implies $u-v \equiv 0$ if $T$ is small enough.

Define the energy of a solution $u \in C\left([0, T] ; H_{0}^{1}(\Omega)\right)$ as

$$
E(t)=\frac{1}{2} \int_{\Omega} a\left(\nabla^{b} u, \nabla^{b} u\right) d x+\frac{1}{2} \int_{\Omega} c(x)|u|^{2} d x+\int_{\Omega} F(u) d x
$$

Theorem 7.2 (Global existence in $H^{1}$ ). Let $n \geq 3$ and assume the coefficients of $L$ satisfy

$b^{2}+|\nabla \cdot b| \in L_{l o c}^{2}, \quad c \in L^{\frac{n}{2}, 1}, \quad\|a-I\|_{L^{\infty}}+\left\||b|+\left|a^{\prime}\right|\right\|_{L^{n, \infty}}+\left\|b^{\prime}\right\|_{L^{\frac{n}{2}, \infty}}+\left\|c_{-}\right\|_{L^{\frac{n}{2}, 1}}<\epsilon$.

Assume $f(u)$ satisfies the conditions (7.2) of the previous result, and in addition it is gauge invariant (1.11) with $F(r)=\int_{0}^{r} f(s) d s \geq 0$ for $s \in \mathbb{R}$. Moreover, assume condition $(S)$ holds.

Then, if $\epsilon$ is sufficiently small, for all initial data $u_{0} \in H_{0}^{1}(\Omega)$ problem (7.1) has a unique global solution $u \in C \cap L^{\infty}\left(\mathbb{R} ; H_{0}^{1}(\Omega)\right)$. In addition the solution has constant energy $E(t) \equiv E(0)$ for all $t \in \mathbb{R}$.

Proof. Since the lifespan of the local solution only depends on the $H^{1}$ norm of the data, in order to prove the claim it is sufficient to prove that the energy $E(t)$ of the solution is conserved. Indeed, $E(t)$ controls the $H^{1}$ norm of $u$, and then global existence follows from a standard continuation argument.

Let $e(u)$ be the energy density

$$
e(u)(t, x)=\frac{1}{2} a(x) \nabla^{b} u \cdot \overline{\nabla^{b} u}+\frac{1}{2} c(x)|u|^{2}+F(u)
$$

so that $E(t)=\int_{\Omega} e(u) d x$. By gauge invariance and the definition of $F$ we have $\partial_{t} F(u)=\partial_{t} \int_{0}^{|u|} f(s) d s=\Re\left(f(|u|) \frac{u}{|u|} \bar{u}_{t}\right)=\Re\left(f(u) \bar{u}_{t}\right)$. If the function $u$ satisfies $u(t) \in H^{2}(\Omega)$, we can write

$$
\partial_{t} e(u)+\nabla \cdot\left\{\Re \overline{u_{t}} a(x) \nabla^{b} u\right\}=\Re \overline{u_{t}}\left(i u_{t}-L u+f(u)\right) \equiv 0
$$

and integrating on $\Omega$, since $\left.u_{t}\right|_{\partial \Omega}=0$ by the Dirichlet boundary conditions, we obtain that $E(u)(t) \equiv E(u)(0)$ is constant in time.

Since we know only $u(t) \in H_{0}^{1}(\Omega)$, in order to use (7.6) we need a regularization procedure; we use the operators $J_{\epsilon}$ constructed at the end of Section 6 . Thus we define $u_{\epsilon}=J_{\epsilon} u$ and note that $u_{\epsilon}$ belongs to $C_{T} H^{2}(\Omega)$ and satisfies

$$
i \partial_{t} u_{\epsilon}-L u_{\epsilon}+J_{\epsilon} f(u)=0 .
$$


Using (7.6) we obtain, after an integration on $\left[t_{1}, t_{2}\right] \times \Omega$, with $0 \leq t_{1}<t_{2} \leq T$,

$$
\left.\int_{\Omega} e\left(u_{\epsilon}\right)\right|_{t_{1}} ^{t_{2}} d x=\Re \int_{t_{1}}^{t_{2}} \int_{\Omega} \partial_{t} \overline{u_{\epsilon}} \cdot\left(f\left(u_{\epsilon}\right)-J_{\epsilon} f(u)\right) d x d t .
$$

Substituting $\partial_{t} u_{\epsilon}$ from the equation and using the Cauchy-Schwartz inequality and the assumption $a_{j k} \in L^{\infty}$ we get

$$
\left|\int_{\Omega} e\left(u_{\epsilon}\right)\right|_{t_{1}}^{t_{2}} d x \mid \lesssim \int_{t_{1}}^{t_{2}}\left[\phi_{\epsilon}(t)+\psi_{\epsilon}(t)+\chi_{\epsilon}(t)\right] d t
$$

where

$$
\begin{gathered}
\phi_{\epsilon}=\int_{\Omega}\left|\nabla^{b} u_{\epsilon}\right| \cdot\left|\nabla^{b}\left(f\left(u_{\epsilon}\right)-J_{\epsilon} f(u)\right)\right| d x, \quad \psi_{\epsilon}(t)=\int_{\Omega}\left|J_{\epsilon} f(u)\right| \cdot\left|f\left(u_{\epsilon}\right)-J_{\epsilon} f(u)\right| d x . \\
\chi_{\epsilon}(t)=\int_{\Omega}|c|\left|u_{\epsilon}\right| \cdot\left|f\left(u_{\epsilon}\right)-J_{\epsilon} f(u)\right| d x
\end{gathered}
$$

Since $u_{\epsilon} \rightarrow u$ in $H_{0}^{1}$ and hence by Sobolev embedding in $L^{\gamma+1}$, we see that $E\left(u_{\epsilon}\right) \rightarrow$ $E(u)$. Thus to conclude the proof it is sufficient to show that the right hand side of (7.7) tends to 0 as $\epsilon \rightarrow 0$, possibly through a subsequence; to this end we shall apply dominated convergence on the interval $[0, T]$.

Consider first the case $n \geq 4$, so that $\gamma+1<n$. We prepare a few additional inequalities:

$$
\left\|\nabla u_{\epsilon}\right\|_{L^{\gamma+1}} \simeq\left\|(-L)^{\frac{1}{2}} J_{\epsilon} u\right\|_{L^{\gamma+1}}=\left\|J_{\epsilon}(-L)^{\frac{1}{2}} u\right\|_{L^{\gamma+1}} \lesssim\left\|(-L)^{\frac{1}{2}} u\right\|_{L^{\gamma+1}} \simeq\|\nabla u\|_{L^{\gamma+1}}
$$

by the $L^{p}$ boundedness of $J_{\epsilon}$ and (6.8) for $\sigma=1 / 2$. By Hölder and Sobolev inequalities in Lorentz spaces, using $b \in L^{n, \infty}$, we have also

$$
\left\|b u_{\epsilon}\right\|_{L^{\gamma+1}} \lesssim\left\|u_{\epsilon}\right\|_{L^{q, \gamma+1}} \lesssim\left\|\nabla u_{\epsilon}\right\|_{L^{\gamma+1}} \lesssim\|\nabla u\|_{L^{\gamma+1}}, \quad \frac{1}{\gamma+1}=\frac{1}{n}+\frac{1}{q}
$$

and summing the two

$$
\left\|\nabla^{b} u_{\epsilon}\right\|_{L^{\gamma+1}} \lesssim\|\nabla u\|_{L^{\gamma+1}}
$$

Thus we have

$$
\left.\phi_{\epsilon}(t) \lesssim\|\nabla u\|_{L^{\gamma+1}} \| \nabla\left(f\left(u_{\epsilon}\right)-J_{\epsilon} f(u)\right)\right)\left\|_{L^{\frac{\gamma+1}{\gamma}}} \lesssim\right\| \nabla u\left\|_{L^{\gamma+1}}^{2}\right\| u \|_{L^{\gamma+1}}^{\gamma-1}=: \phi(t) .
$$

Note that $\phi \in L^{1}(0, T)$ since

$$
\int_{0}^{T} \phi d t \leq\|\nabla u\|_{L_{T}^{2} L^{\gamma+1}}^{2}\|u\|_{L^{\infty} L^{\gamma+1}}^{\gamma-1}
$$

and $\nabla u \in L_{T}^{p} L^{\gamma+1}$ for some $p>2$ by Strichartz estimates, while $u \in C_{T} H_{0}^{1} \hookrightarrow$ $L_{T}^{\infty} L^{\gamma+1}$ by Sobolev embedding. For $\psi_{\epsilon}$ we have easily

$$
\psi_{\epsilon}(t) \lesssim\|u\|_{L^{2 \gamma}}^{2 \gamma}=: \psi(t),
$$

and by the interpolation and Sobolev inequalities

$$
\|u\|_{L^{2 \gamma}}^{2 \gamma} \leq\|u\|_{L^{\gamma+1}}^{2 \gamma-\sigma}\|u\|_{L^{\frac{n(\gamma+1)}{n-(\gamma+1)}}}^{\sigma} \lesssim\|u\|_{L^{\gamma+1}}^{2 \gamma-\sigma}\|\nabla u\|_{L^{\gamma+1}}^{\sigma}, \quad \sigma=\frac{\gamma-1}{\gamma+1} n
$$

so that

$$
\int_{0}^{T} \psi d t \lesssim\|u\|_{L_{T}^{\infty} L^{\gamma+1}}^{2 \gamma-\sigma}\|\nabla u\|_{L_{T}^{\sigma} L^{\gamma+1}}^{\sigma}
$$

and again we obtain $\psi \in L^{1}(0, T)$ since $0<\sigma<2$ for $1<\gamma<\frac{n+2}{n-2}$. As to $\chi_{\epsilon}$, recalling that $|c|^{\frac{1}{2}} \in L^{n, \infty}$, we can write

$\left\|c u_{\epsilon} J_{\epsilon} f(u)\right\|_{L^{1}} \leq\left\||c|^{\frac{1}{2}} u_{\epsilon}\right\|_{L^{\gamma+1}}\left\||c|^{\frac{1}{2}} J_{\epsilon} f(u)\right\|_{L^{\frac{\gamma+1}{\gamma}}} \lesssim\|\nabla u\|_{L^{\gamma+1}}\left\|\nabla J_{\epsilon} f(u)\right\|_{L^{\frac{\gamma+1}{\gamma}}} \lesssim \phi(t)$ proceeding as in the estimate of $b u_{\epsilon}$; the term $c u_{\epsilon} f\left(u_{\epsilon}\right)$ is similar. Thus the sequences $\phi_{\epsilon}, \psi_{\epsilon}, \chi_{\epsilon}$ are dominated. Moreover, it is easy to check, using exactly the previous estimates and properties (6.11), (6.16), (6.17) and (6.18), that for a.e. $t \in[0, T]$ one has $\phi_{\epsilon}(t), \psi_{\epsilon}(t), \chi_{\epsilon}(t) \rightarrow 0$ as $\epsilon \rightarrow 0$.

In the case $n=3$, the quantity $\gamma+1$ is in the range $2 \leq \gamma+1<6$ and can be larger than $n$. The previous computations work fine for $1 \leq \gamma<2$; when $2 \leq \gamma<5$ 
it is not difficult to modify the choice of indices so to use only the allowed Strichartz norms. For the estimate of $\phi_{\epsilon}(t)$ we can write for $\frac{1}{4}<\epsilon<\frac{1}{2}$

$$
\phi_{\epsilon}(t) \lesssim\|\nabla u\|_{L^{\frac{3}{1+\epsilon}}}^{2}\|u\|_{L^{\frac{3(\gamma-1)}{1-2 \epsilon}}}^{\gamma-1} \lesssim\|\nabla u\|_{L^{\frac{3}{1+\epsilon}}}^{2}\|\nabla u\|_{L^{\frac{3(\gamma-1)}{\gamma-2 \epsilon}}}^{\gamma-1}=: \phi(t)
$$

by Hölder and Sobolev inequalitites, and hence

$$
\int_{0}^{T} \phi(t) d t \leq\|\nabla u\|_{L_{T}^{2} \frac{4}{1-2 \epsilon}}^{\frac{3}{1+\epsilon}}\|\nabla u\|_{L_{T}^{\frac{2(\gamma-1)}{1+2 \epsilon}}}^{\gamma} L^{\frac{3(\gamma-1)}{\gamma-2 \epsilon}} .
$$

Notice that the first factor is an (allowed) Strichartz norm, while the second factor can be estimated by Hölder inequality in time with the Strichartz norm

$$
\|\nabla u\|_{L_{T}^{\gamma-3+4 \epsilon}}^{\frac{4(\gamma-1)}{\gamma-1}} L^{\frac{3(\gamma-1)}{\gamma-2 \epsilon}},
$$

(which is allowed and meaningful for $\frac{1}{4}<\epsilon<\frac{1}{2}$ ) since the condition $\frac{4(\gamma-1)}{\gamma-3+4 \epsilon}>$ $\frac{2(\gamma-1)}{1+2 \epsilon}$ is equivalent to $\gamma<5$. The reamining estimates can be modified in a similar way; we omit the details.

The next Proposition is the crucial step in the proof of scattering. We follow the simpler approach to scattering developed in [33] and [6]. We prefer this to the more technical method of [31], which could also be used here.

Proposition 7.3. Let $n \geq 3$, and consider Problem (7.1) under the assumptions of Theorem 1.4 if $n \geq 4$ or of Theorem 1.5 if $n=3$. Then any solution $u \in$ $C \cap L^{\infty}\left(\mathbb{R} ; H_{0}^{1}(\Omega)\right)$ satisfies

$$
\lim _{t \rightarrow \pm \infty}\|u(t, \cdot)\|_{L^{r}}=0 \quad \text { for all } \quad 2<r<\frac{2 n}{n-2} .
$$

Proof. We consider only the case $t \rightarrow+\infty$; the proof in the case $t \rightarrow-\infty$ is identical. It is enough to prove (7.8) for $r=2+\frac{4}{n}$, i.e.,

$$
\lim _{t \rightarrow+\infty}\|u(t, \cdot)\|_{L^{2+\frac{4}{n}}}=0 .
$$

Indeed, the $H^{1}$ norm of $u$ is bounded for $t \in \mathbb{R}$, so that by Sobolev inequality we have

$$
\|u(t, \cdot)\|_{L^{\frac{2 n}{n-2}}}+\|u(t, \cdot)\|_{L^{2}} \lesssim\|u(t, \cdot)\|_{H^{1}}+\|u(t, \cdot)\|_{L^{2}} \leq C
$$

with $C$ independent of $t$, and interpolating with (7.9) we obtain the full claim (7.8).

Assume by contradiction that there exist an $\epsilon_{0}>0$ and a sequence of times $t_{k} \uparrow+\infty$ such that for all $k$

$$
\left\|u\left(t_{k}, \cdot\right)\right\|_{L^{2+\frac{4}{n}}} \geq \epsilon_{0} .
$$

Denote with $Q_{R}(x)$ the intersection with $\Omega$ of the cube of side $R$ and center $x$ (with sides parallel to the axes). By interpolation in $L^{p}$ spaces and Sobolev embedding, we have for all $v \in H_{0}^{1}(\Omega)$ and $x \in \Omega$

$$
\|v\|_{L^{2+\frac{4}{n}}\left(Q_{1}(x)\right)}^{2+\frac{4}{2}} \leq\|v\|_{L^{\frac{2 n}{n-2}}\left(Q_{1}(x)\right)}^{2} \cdot\|v\|_{L^{2}\left(Q_{1}(x)\right)}^{\frac{4}{n}} \lesssim\|v\|_{H^{1}\left(Q_{1}(x)\right)}^{2} \cdot\|v\|_{L^{2}\left(Q_{1}(x)\right)}^{\frac{4}{n}}
$$

which implies, for all $x \in \Omega$,

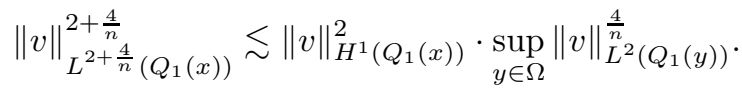

Choosing a sequence of centers $x \in \Omega$ such that the cubes $Q_{1}(x)$ cover $\Omega$ and are almost disjoint, and summing over all cubes, we obtain the inequality

$$
\|v\|_{L^{2+\frac{4}{n}(\Omega)}}^{2+\frac{4}{n}} \lesssim\|v\|_{H^{1}(\Omega)}^{2} \cdot \sup _{x \in \Omega}\|v\|_{L^{2}\left(Q_{1}(x)\right)}^{\frac{4}{2}} .
$$


Combining (7.12) with the energy bound (7.10) and recalling (7.11), we obtain that there exists a sequence of points $x_{k} \in \Omega$ such that

$$
\left\|u\left(t_{k}, \cdot\right)\right\|_{L^{2}\left(Q_{1}\left(x_{k}\right)\right)} \geq \epsilon_{1}>0 .
$$

We claim that we can find $\bar{t}>0$ such that

$$
\|u(t, \cdot)\|_{L^{2}\left(Q_{2}\left(x_{k}\right)\right)} \geq \epsilon_{1} / 2 \text { for all } t \in\left(t_{k}, t_{k}+\bar{t}\right) .
$$

Indeed, consider a cut-off function $\chi \in C_{c}^{\infty}\left(\mathbb{R}^{n}\right)$ such that $\chi(x)=1$ on the cube of side 1 with center $x_{k}$, and $\chi(x)=0$ outside the cube of side 2 with center $x_{k}$. We integrate the elementary identity

$$
\frac{d}{d t}\left[\chi(x)|u(t, x)|^{2}\right]=2 \chi(x) \nabla \cdot\left\{\Im\left[a(x) \nabla^{b} u(t, x) \bar{u}(t, x)\right]\right\}
$$

on $\Omega$ and we obtain, for all $t \in \mathbb{R}$,

$$
\begin{aligned}
\left.\left|\frac{d}{d t} \int_{\Omega} \chi(x)\right| u(t, x)\right|^{2} d x \mid & \lesssim\left|\int_{\Omega} \nabla \chi(x) \cdot \Im\left[a(x) \nabla^{b} u(t, x) \bar{u}(t, x)\right]\right| \\
& \lesssim\|u(t, \cdot)\|_{L^{2}(\Omega)}\left\|\nabla^{b} u(t, \cdot)\right\|_{L^{2}(\Omega)} \\
& \leq\|u(0, \cdot)\|_{L^{2}(\Omega)} \sup _{t \in \mathbb{R}}\|\nabla u(t, \cdot)\|_{L^{2}(\Omega)}=: \bar{C}<+\infty
\end{aligned}
$$

where we used (2.15). This implies

$$
\int_{Q_{2}\left(x_{k}\right)}|u(t, x)|^{2} d x \geq \int_{Q_{1}\left(x_{k}\right)}\left|u\left(t_{k}, x\right)\right|^{2} d x-\bar{C}\left|t-t_{k}\right|,
$$

whence (7.13) follows provided that we choose $\bar{t}>0$ such that $\epsilon_{1}^{2}-\bar{C} \bar{t}>\epsilon_{1}^{2} / 4$. Note, by passing to a subsequence, we can assume the intervals $\left(t_{k}, t_{k}+\bar{t}\right)$ to be disjoint.

If $n \geq 4$, we get

$$
\iint_{\Omega \times \Omega} \frac{|u(t, x)|^{2}|u(t, y)|^{2}}{|x-y|^{3}} d x d y d t \gtrsim \sum_{k} \int_{t_{k}}^{t_{k}+\bar{t}} \int_{Q_{2}\left(x_{k}\right) \times Q_{2}\left(x_{k}\right)}|u(t, x)|^{2}|u(t, y)|^{2} d x d y d t=\infty .
$$

but this is in contradiction with (1.23), since $u \in L^{\infty}\left(\mathbb{R}, H_{0}^{1}(\Omega)\right)$, and this concludes the proof in this case. On the other hand, if $n=3$, from (7.13) we get that

$$
\|u\|_{L^{4}\left(\left(t_{k}, t_{k}+\bar{t}\right) \times Q_{2}\left(x_{k}\right)\right)}^{4} \geq C \epsilon_{1}^{4} \bar{t},
$$

which is in contradiction with (1.24).

By fairly standard arguments, property (7.8) implies that the Strichartz norms of a global $H^{1}$ solutions are bounded, and scattering follows. The only limitation here is the requirement $q_{1}<n$ in Assumption (S), which is effective only in dimension $n=3,4$. We sketch the arguments for the sake of completeness:

Proposition 7.4. Let $u \in C \cap L^{\infty}\left(\mathbb{R} ; H_{0}^{1}(\Omega)\right)$ be a solution to Problem (7.1) under the assumptions of Theorem 1.4 if $n \geq 4$ and under the assumptions of Theorem 1.5 if $n=3$. Moreover, assume that $(\bar{S})$ holds and that $\gamma>1+\frac{4}{n}$. Then for every admissible pair $(p, q)$ we have $u \in L^{p} L^{q}$, and for every admissible pair $(p, q)$ with $q<n$ we have $\nabla u \in L^{p} L^{q}$.

Proof. We consider in detail the case $n \geq 4$, where $\gamma+1<n$. For the case $n=3$ in the range $2 \leq \gamma<6$, the following arguments can be easily modified as in the last part of the proof in Theorem 7.2. Note that we know that the Strichartz norms are finite on bounded time intervals, and we only need to prove an uniform bound as the time interval invades $\mathbb{R}$.

We use the notation $L_{T, t}^{p} L^{q}:=L^{p}\left(T, t ; L^{q}(\Omega)\right)$ for $t>T$. By Strichartz estimates on the time interval $[T, t]$ for the admissible couple $(p, \gamma+1)$ where $p=\frac{4}{n} \frac{\gamma+1}{\gamma-1}$ we 
have

$$
\begin{aligned}
\|u\|_{L_{T, t}^{p} L^{\gamma+1}} & \lesssim\|u(T)\|_{L^{2}}+\|f(u)\|_{L_{T, t}^{p^{\prime}} L(\gamma+1)^{\prime}} \\
& \lesssim\|u(T)\|_{L^{2}}+\|\| u\left\|_{L^{\gamma+1}}^{\gamma}\right\|_{L_{T, t}^{p^{\prime}}}
\end{aligned}
$$

since $|f(u)| \lesssim|u|^{\gamma}$ and $(\gamma+1)^{\prime} \gamma=\gamma+1$. The condition $\gamma>1+\frac{4}{n}$ is equivalent to $\gamma>\frac{p}{p^{\prime}}$, thus we can continue the estimate as follows:

$$
\begin{aligned}
& \lesssim\|u(T)\|_{L^{2}}+\|u\|_{L_{T, L^{\prime}}^{\infty}}^{\gamma-\frac{p}{p^{\prime}}}\|\| u\left\|_{L^{\gamma+1}}^{\frac{p}{p}}\right\|_{L_{T, t}^{p^{\prime}}} \\
& \leq\|u(T)\|_{L^{2}}+\|u\|_{L_{T, \infty}^{\infty}}^{\gamma-\frac{p}{p^{\prime}}} L^{\frac{p}{\gamma+1}}\|u\|_{L_{T, t}^{p^{\prime}} L^{\gamma+1}}^{\frac{p}{p^{\prime}}} .
\end{aligned}
$$

By Proposition 7.3 we know that $o(T)=\|u\|_{L_{T, \infty}^{\infty} L^{\gamma+1}} \rightarrow 0$ as $T \rightarrow \infty$. Thus the function $\phi(t):=\|u\|_{L_{T, t}^{p} L^{\gamma+1}}$ satisfies an inequality of the form $\phi(t) \leq C+$ $o(T) \phi(t)^{\frac{p}{p^{\prime}}}$. Taking $T$ large enough, an easy continuity argument shows that $\phi(t)$ is bounded for all $t>T$. This proves that $u \in L^{p} L^{\gamma+1}$. Now we notice that in the previous computations we have also proved that $f(u) \in L^{p^{\prime}} L^{(\gamma+1)^{\prime}}$, and using again Strichartz estimates we conclude that $u \in L^{r} L^{q}$ for all admissible $(r, q)$.

The estimate of $\nabla u$ is similar:

$$
\begin{aligned}
\|\nabla u\|_{L_{T, t}^{p} L^{\gamma+1}} & \lesssim\|\nabla u(T)\|_{L^{2}}+\|\nabla f(u)\|_{L_{T, t}^{p^{\prime}}(\gamma+1)^{\prime}} \\
& \lesssim\|\nabla u(T)\|_{L^{2}}+\|\| u\left\|_{L^{\gamma+1}}^{\gamma-1}\right\| \nabla u\left\|_{L^{\gamma+1}}\right\|_{L_{T, t}^{p^{\prime}}}
\end{aligned}
$$

since $\left|f^{\prime}(u)\right| \lesssim|u|^{\gamma-1}$, and as before, using Hölder inequality,

$$
\begin{aligned}
& \lesssim\|\nabla u(T)\|_{L^{2}}+\|u\|_{L_{T, \infty}^{\infty} L^{\gamma+1}}^{\gamma-\frac{p}{p^{\prime}}}\|\| u\left\|_{L^{\gamma+1}}^{\frac{p}{p^{\gamma}}-1}\right\| \nabla u\left\|_{L^{\gamma+1}}\right\|_{L_{T, t}^{p^{\prime}}} \\
& \lesssim\|\nabla u(T)\|_{L^{2}}+\|u\|_{L_{T, \infty}^{\infty} \frac{p}{p^{\prime}}}^{\frac{p}{\gamma+1}}\|u\|_{L_{T, t}^{p} L^{\gamma+1}}^{\frac{p}{p^{\prime}}-1}\|\nabla u\|_{L_{T, t}^{p} L^{\gamma+1}} .
\end{aligned}
$$

By the bound already proved, this implies

$$
\|\nabla u\|_{L_{T, t}^{p} L^{\gamma+1}} \lesssim\|\nabla u(T)\|_{L^{2}}+o(T)\|\nabla u\|_{L_{T, t}^{p} L^{\gamma+1}}
$$

and taking $T$ large enough we obtain the claim.

We can now conclude the proof of Theorem 1.7. Part (i) is Theorem 7.2. Scattering is an immediate consequence of the a priori bounds of the Strichartz norms proved in Proposition 7.4. We briefly sketch the main steps of the proof which are completely standard, in the case $t \rightarrow+\infty$; the case $t \rightarrow-\infty$ is identical.

To construct the wave operator (claim (ii) of the Theorem), given $u_{+} \in H_{0}^{1}(\Omega)$, we consider the integral equation

$$
u(t):=e^{-i t L} u_{+}+i \int_{t}^{\infty} e^{-i(t-s)} f(u(s)) d s
$$

and we look for a solution defined on $[T, \infty)$, for $T$ sufficiently large. Using Strichartz estimates with the same choice of indices as in the proof of local existence, and noticing that the Strichartz norms of $e^{-i t L} u_{+}$are arbitrarily small for $T$ large, by a fixed point approach we construct a solution $u \in C \cap L^{\infty}\left([T,+\infty), H_{0}^{1}(\Omega)\right)$ to (7.15). This is also a solution to the Schrödinger equation in (7.1), and thanks to the global existence result, $u$ can be extended to a solution $u \in C \cap L^{\infty}\left(\mathbb{R}, H_{0}^{1}(\Omega)\right)$ defined for all $t \in \mathbb{R}$. We can then choose $u_{0}=u(0)$. Uniqueness follows by a similar argument: if two solutions $u_{1}, u_{2}$ of (7.1), with possibly different data, have the same asymptotic behaviour i.e. $\left\|u_{1}(t)-u_{2}(t)\right\|_{H^{1}} \rightarrow 0$ as $t \rightarrow+\infty$, then they both solve (7.15), and the previous fixed point argument implies $u_{1}(t)=u_{2}(t)$ for $t$ large. Then $u_{1} \equiv u_{2}$ by global uniqueness. 
To prove asymptotic completeness (claim (iii) of the Theorem), we fix a $u_{0} \in$ $H_{0}^{1}(\Omega)$ and let $u(t)$ be the corresponding global solution to Problem (7.1). Then we define $v(t)=e^{i t L} u(t)$ and note that

$$
v(t)=u_{0}+i \int_{0}^{t} e^{i s L} f(u(s)) d s .
$$

Note that $\left\|e^{i t L} \phi\right\|_{L 2}=\|\phi\|_{L^{2}}$ by the unitarity of $e^{i t L}$; moreover, since $(-L \phi, \phi)_{L^{2}} \simeq$ $\|\phi\|_{\dot{H}^{1}}^{2}$, we have $\left\|e^{i t L} \phi\right\|_{\dot{H}^{1}}^{2} \simeq\left(-L e^{i t L} \phi, e^{i t L} \phi\right)_{L^{2}} \simeq\|\phi\|_{\dot{H}^{1}}$, and in conclusion we get

$$
\left\|e^{i t L} \phi\right\|_{H^{1}} \simeq\|\phi\|_{H^{1}} \quad \forall \phi \in H_{0}^{1}(\Omega)
$$

with constants uniform in $t$. Thus for $0<\tau<t$ we can write

$$
\|v(t)-v(\tau)\|_{H^{1}} \simeq\left\|e^{-i t L}(v(t)-v(\tau))\right\|_{H^{1}}=\left\|\int_{\tau}^{t} e^{-i(t-s) L} f(u) d s\right\|_{L_{t}^{\infty} H^{1}}
$$

and by Strichartz estimates, Hölder inequality and interpolation, we get

$$
\|v(t)-v(\tau)\|_{H^{1}} \lesssim\|f(u)\|_{L_{\tau, t}^{p^{\prime} W^{1,(\gamma+1)^{\prime \prime}}}}
$$

where $p=\frac{4}{n} \frac{\gamma+1}{\gamma-1}$; this choice is always possible in dimension $n \geq 4$; in dimension $n=$ 3 for the range $2 \leq \gamma<6$ one needs to modify the choice as in the proof of Theorem 7.2. By Proposition 7.4 we know that the Strichartz norms of $u$ are bounded, and by the same argument used in that proof we see that $f(u) \in L^{p^{\prime}} W^{1,(\gamma+1)^{\prime}}$. As a consequence, the right hand side of the previous inequality can be made arbitrarily small provided $t, \tau$ are large enough. We deduce that $v(t)$ converges in $H_{0}^{1}(\Omega)$ as $t \rightarrow+\infty$ to a limit $u_{+}$, and finally

$$
\left\|u(t)-e^{-i t L} u_{+}\right\|_{H^{1}} \simeq\left\|v(t)-u_{+}\right\|_{H^{1}} \rightarrow 0
$$

as claimed.

\section{Strichartz estimates}

Throughout this section, $\Omega=\mathbb{R}^{n}$ and $L$ is the selfadjoint operator on $L^{2}\left(\mathbb{R}^{n}\right)$ defined in Proposition 6.1. We look for sufficient conditions on the coefficients $a, b, c$ in order to have Strichartz estimates on $\mathbb{R}^{n}$ for the flow $e^{i t L}$

$$
\begin{gathered}
\left\|e^{i t L} u_{0}\right\|_{L^{p_{1} L^{q_{1}}}} \lesssim\left\|u_{0}\right\|_{L^{2}}, \\
\left\|\int_{0}^{t} e^{i(t-s) L} F d s\right\|_{L^{p_{1} L^{q_{1}}}} \lesssim\|F\|_{L^{p_{2}^{\prime} L^{q_{2}^{\prime}}}}
\end{gathered}
$$

and for the derivative of the flow $\nabla e^{i t L}$

$$
\begin{gathered}
\left\|\nabla e^{i t L} u_{0}\right\|_{L^{p_{1} L^{q_{1}}}} \lesssim\left\|\nabla u_{0}\right\|_{L^{2}}, \\
\left\|\nabla \int_{0}^{t} e^{i(t-s) L} F d s\right\|_{L^{p_{1}} L^{q_{1}}} \lesssim\|\nabla F\|_{L^{p_{2}^{\prime}} L^{q_{2}^{\prime}}}
\end{gathered}
$$

for admissible couples of indices $\left(p_{j}, q_{j}\right)$. Recall that admissible couples $(p, q)$ satisfy $p \in[2, \infty], q \in\left[2, \frac{2 n}{n-2}\right]$ with $\frac{2}{p}+\frac{n}{q}=\frac{n}{2}$ and the endpoint is the couple $\left(2, \frac{2 n}{n-2}\right)$.

We shall derive the estimates of the first kind by combining Tataru's results in [32] with our smoothing estimates. On the other hand, in order to deduce (8.3), (8.4) we shall use the equivalence of Sobolev norms proved in Proposition 6.3. The following result is a direct application of [32]:

Theorem 8.1. Let $n \geq 3$. Assume the coefficients $a, b, c$ of L satisfy

$$
|a-I|+\langle x\rangle\left(\left|a^{\prime}\right|+|b|\right)+\langle x\rangle^{2}\left(\left|a^{\prime \prime}\right|+\left|b^{\prime}\right|+|c|\right) \leq \epsilon\langle x\rangle^{-\delta}
$$

for some $\epsilon, \delta>0$. If $\epsilon$ is sufficiently small, the flow $e^{i t L}$ satisfies the Strichartz estimates (8.1), (8.2) for all admissible couples $\left(p_{j}, q_{j}\right), j=1,2$, including the endpoint. 
Proof. We rewrite $L$ as the sum of $A u=\nabla \cdot(a \nabla u)$ plus lower order terms

$$
L u=A u+2 i a(\nabla u, b)+i \partial_{j}\left(a_{j k} b_{k}\right) u-a(b, b) u-c(x) u .
$$

Define the norm

$$
\|v\|_{Z}=\|v\|_{L^{\infty}(|x| \leq 1)}+\sum_{j \geq 1}\|v\|_{L^{\infty}\left(2^{j-1} \leq|x| \leq 2^{j}\right)} .
$$

By Theorem 4 and Remarks 6 and 7 in [32], if $a, b, c$ satisfy

$$
\begin{gathered}
\left\|\langle x\rangle^{2}\left|a^{\prime \prime}(x)\right|\right\|_{Z}+\left\|\langle x\rangle\left|a^{\prime}(x)\right|\right\|_{Z}+\||a(x)-I|\|_{Z} \leq \epsilon, \\
\left\|\langle x\rangle^{2} \partial_{m}\left(a_{j k} b_{k}\right)\right\|_{Z}+\left\|\langle x\rangle a_{j k} b_{k}\right\|_{Z} \leq \epsilon, \\
\left\|\langle x\rangle^{2}\left[\left|\partial_{j}\left(a_{j k} b_{k}\right)\right|+|a(b, b)|+|c(x)|\right]\right\|_{Z} \leq \epsilon
\end{gathered}
$$

for $\epsilon$ small enough, then the linear flow $e^{i t L}$ satisfies the full set of Strichartz estimates (8.1), (8.2). It is immediate to check that condition (8.5) implies (8.6)(8.8).

Combining the previous Theorem with our smoothing estimate (Corollary 1.3) we cover the case of repulsive electric potentials with a large positive part:

Theorem 8.2. Let $n \geq 3$. Assume the coefficients $a, b$ of $L$ satisfy

$$
|a-I|+\langle x\rangle\left(\left|a^{\prime}\right|+|b|\right)+\langle x\rangle^{2}\left(\left|a^{\prime \prime}\right|+\left|b^{\prime}\right|\right)+\langle x\rangle^{3}\left|a^{\prime \prime \prime}\right| \leq \epsilon\langle x\rangle^{-\delta}
$$

while the potential $c(x)$ satisfies

$$
-\epsilon\langle x\rangle^{-2} \leq c(x) \leq C_{+}^{2}\langle x\rangle^{-2}, \quad\langle x\rangle^{1+\delta} c \in L^{n}
$$

and the repulsivity condition

$$
a(x) x \cdot \nabla c(x) \leq \epsilon|x|^{-1}\langle x\rangle^{-1-\delta}
$$

for some $\epsilon, \delta, C_{+}>0$. If $\epsilon$ is sufficiently small, the flow $e^{i t L}$ satisfies the homogeneous Strichartz estimates (8.1) for all admissible couples, and the inhomogeneous estimates (8.2) for all couples with the exception of the endpoint-endpoint case.

Proof. By Theorem 8.1, Strichartz estimates are valid for the flow $e^{i t L_{0}}$ with $c=0$. The complete flow $u=e^{i t L} u_{0}$ satisfies the equation $i u_{t}+L_{0} u=c u$, hence it can be written

$$
u=e^{i t L} u_{0}=e^{i t L_{0}} u_{0}-i \int_{0}^{t} e^{i(t-s) L_{0}}(c u) d s
$$

so that, by the previous result,

$$
\|u\|_{L^{p} L^{q}} \lesssim\left\|u_{0}\right\|_{L^{2}}+\|c u\|_{L^{2} L^{\frac{2 n}{n+2}}}
$$

for all admissible couples $(p, q)$. By Hölder inequality we have

$$
\|c u\|_{L^{2} L^{\frac{2 n}{n+2}}} \lesssim\left\|\langle x\rangle^{1+\delta} c\right\|_{L^{n}}\left\|\langle x\rangle^{-1-\delta} u\right\|_{L^{2} L^{2}}
$$

and the homogeneous estimate will be proved if we can prove the estimate

$$
\left\|\langle x\rangle^{-1-\delta} u\right\|_{L^{2} L^{2}} \lesssim\left\|u_{0}\right\|_{L^{2}} .
$$

Indeed, the assumptions of Corollary 1.3 are satisfied by $L$; in particular, the gaussian upper bound for the heat flow $e^{i t L}$ is valid for general $L^{\infty}$ coefficients (see Theorem 5.4 in [26] or [27]). Thus (8.12) follows from inequality (1.21) and we obtain the full set of homogeneous Strichartz estimates for the flow $e^{i t L}$.

To prove inhomogeneous estimates it is sufficient to apply a standard $T T^{*}$ argument combined with the Christ-Kiselev lemma, and this gives (8.2) with the exception of the endpoint-endpoint case. 
We conclude the section by proving the estimates for the flow $\nabla e^{i t L}$, which are now a straightforward consequence of the previous results. Note that the application of Proposition 6.3 imposes an additional condition $q_{1}<n$, which is restrictive only in dimensions $n=3$ and 4 .

Corollary 8.3. Let $n \geq 3$. Estimates (8.3), (8.4) hold for the flow $\nabla e^{i t L}$, for all admissible couples $\left(p_{j}, q_{j}\right), j=1,2$, provided $q_{1}<n$ and the coefficients $a, b, c$ of $L$ satisfy either assumption (8.5), or assumptions (8.9), (8.10), (8.11), provided $\epsilon$ is small enough.

Proof. In both cases we see that the assumptions of Proposition 6.3 are satisfied. In particular, in the second case the smallness of the $L^{\frac{n}{2}, 1}$ norm of $c_{-}$follows from the fact that the $L^{n}$ norm of $\langle x\rangle^{1+\delta} c$ is arbitrarily small outside a sufficiently large ball, and inside the ball we have $\left|c_{-}\right| \leq \epsilon$ by condition (8.10).

Now in the first case the assumptions of Theorem 8.1 are satisfied and we can write

$$
\begin{aligned}
\left\|\nabla e^{i t L} u_{0}\right\|_{L^{p_{1}} L^{q_{1}}} & \simeq\left\|(-L)^{\frac{1}{2}} e^{i t L} u_{0}\right\|_{L^{p_{1}} L^{q_{1}}}=\left\|e^{i t L}(-L)^{\frac{1}{2}} u_{0}\right\|_{L^{p_{1}} L^{q_{1}}} \\
& \lesssim\left\|(-L)^{\frac{1}{2}} u_{0}\right\|_{L^{2}} \simeq\left\|\nabla u_{0}\right\|_{L^{2}}
\end{aligned}
$$

by a repeated application of (6.8) for $\sigma=\frac{1}{2}$. The proof of the remaining claims is identical.

\section{REFERENCES}

[1] Dean Baskin, Jeremy L. Marzuola, and Jared Wunsch. Strichartz estimates on exterior polygonal domains, 2012.

[2] Matthew D. Blair, Hart F. Smith, and Chris D. Sogge. Strichartz estimates and the nonlinear Schrödinger equation on manifolds with boundary. Mathematische Annalen, 354(4):13971430, 2012.

[3] Federico Cacciafesta. Smoothing estimates for the variable coefficients Schrödinger equation with electromagnetic potentials. Journal of Mathematical Analysis and Applications, 402(1):286-296, 2013.

[4] Federico Cacciafesta and Piero D'Ancona. Weighted $L^{p}$ estimates for powers of selfadjoint operators. Advances in Mathematics, 229(1):501-530, 2012.

[5] Federico Cacciafesta, Piero D'Ancona, and Renato Luca'. Helmholtz and dispersive equations with variable coefficients on exterior domains. (2014).

[6] Biagio Cassano and Mirko Tarulli. $h^{1}$-scattering for systems of $n$-defocusing weakly coupled nls equations in low dimensions. 2014.

[7] Thierry Cazenave. Semilinear Schrödinger equations, volume 10 of Courant Lecture Notes in Mathematics. New York University Courant Institute of Mathematical Sciences, 2003.

[8] J. Colliander, M. Keel, G. Staffilani, H. Takaoka, and Terry Tao. Global existence and scattering for rough solutions of a nonlinear Schrödinger equation on $\mathbb{R}^{3}$. Communications on Pure and Applied Mathematics, 57(8):987-1014, 2004.

[9] James Colliander, Magdalena Czubak, and Jeonghun J. Lee. Interaction Morawetz estimate for the magnetic Schrödinger equation and applications. Advances in Differential Equations, 19(9-10):805-832, 2014.

[10] Piero D'Ancona and Luca Fanelli. $L^{p}$-boundedness of the wave operator for the one dimensional Schrödinger operator. Communications in Mathematical Physics, 268(2):415-438, 2006.

[11] Piero D'Ancona and Luca Fanelli. Strichartz and smoothing estimates of dispersive equations with magnetic potentials. Communications in Partial Differential Equations, 33(4-6):10821112, 2008.

[12] Piero D'Ancona, Luca Fanelli, Luis Vega, and Nicola Visciglia. Endpoint Strichartz estimates for the magnetic Schrödinger equation. Journal of Functional Analysis, 258(10):3227-3240, 2010.

[13] Piero D'Ancona and Vittoria Pierfelice. On the wave equation with a large rough potential. Journal of Functional Analysis, 227(1):30-77, 2005.

[14] Xuan Thinh Duong, El Maati Ouhabaz, and Adam Sikora. Plancherel-type estimates and sharp spectral multipliers. Journal of Functional Analysis, 196(2):443-485, 2002. 
[15] M. Burak Erdoğan, Michael Goldberg, and Wilhelm Schlag. Strichartz and smoothing estimates for Schrödinger operators with almost critical magnetic potentials in three and higher dimensions. 21:687-722, 2009.

[16] Luca Fanelli and Luis Vega. Magnetic virial identities, weak dispersion and Strichartz inequalities. Mathematische Annalen, 344(2):249-278, 2009.

17] Jean Ginibre and Giorgio Velo. Scattering theory in the energy space for a class of nonlinear Schrödinger equations. Journal de Mathématiques Pures et Appliquées. Neuvième Série, 64(4):363-401, 1985.

[18] Loukas Grafakos and Seungly Oh. The kato-ponce inequality, 2013.

[19] Tosio Kato. Wave operators and unitary equivalence. Pacific Journal of Mathematics, 15:171$180,1965$.

[20] Tosio Kato. Wave operators and similarity for some non-selfadjoint operators. Mathematische Annalen, 162:258-279, 1965/1966.

[21] Herbert Leinfelder and Christian G. Simader. Schrödinger operators with singular magnetic vector potentials. Mathematische Zeitschrift, 176:1-19, 1981.

[22] Vitali Liskevich, Hendrik Vogt, and Jürgen Voigt. Gaussian bounds for propagators perturbed by potentials. Journal of Functional Analysis, 238(1):245-277, 2006.

[23] Cathleen S. Morawetz. Time decay for the nonlinear Klein-Gordon equations. 306:291-296, 1968.

[24] Cathleen S. Morawetz. Decay of sloutions of the exterior problem for the wave equation. 28:229-264, 1975.

[25] Richard O'Neil. Convolution operators and L(p, q) spaces. Duke Mathematical Journal, 30:129-142, 1963.

[26] El Maati Ouhabaz. Gaussian upper bounds for heat kernels of second-order elliptic operators with complex coefficients on arbitrary domains. Journal of Operator Theory, 51(2):335-360, 2004.

[27] El Maati Ouhabaz. Analysis of heat equations on domains, volume 31 of London Mathematical Society Monographs Series. Princeton University Press, 2005.

[28] Luc Robbiano and Claude Zuily. Strichartz estimates for Schrödinger equations with variable coefficients. Mémoires de la Société Mathématique de France. Nouvelle Série, (101102):vi+208, 2005.

[29] Igor Rodnianski and Wilhelm Schlag. Time decay for solutions of Schrödinger equations with rough and time-dependent potentials. Inventiones Mathematicae, 155(3):451-513, 2004.

[30] Gigliola Staffilani and Daniel Tataru. Strichartz estimates for a Schrödinger operator with nonsmooth coefficients. Communications in Partial Differential Equations, 27(7-8):1337$1372,2002$.

[31] Terence Tao, Monica Visan, and Xiaoyi Zhang. The nonlinear Schrödinger equation with combined power-type nonlinearities. Communications in Partial Differential Equations, 32(79):1281-1343, 2007.

[32] Daniel Tataru. Parametrices and dispersive estimates for Schrödinger operators with variable coefficients. American Journal of Mathematics, 130(3):571-634, 2008.

[33] Nicola Visciglia. On the decay of solutions to a class of defocusing NLS. Mathematical Research Letters, 16(5-6):919-926, 2009.

[34] Jürgen Voigt. Absorption semigroups, their generators, and Schrödinger semigroups. Journal of Functional Analysis, 67:167-205, 1986.

[35] Kenji Yajima. The $W^{k, p}$-continuity of wave operators for schrödinger operators. iii. evendimensional cases $m \geq 4$. 2(2):311-346, 1995.

[36] Kenji Yajima. $L^{p}$-boundedness of wave operators for two-dimensional Schrödinger operators. 208(1):125-152, 1999.

Biagio Cassano: SAPienZA - Università di Roma, Dipartimento di Matematica, PiAzZAle A. Moro 2, I-00185 Roma, ItAly

E-mail address: cassano@mat.uniroma1.it

Piero D'Ancona: SAPIEnZA - Università di Roma, Dipartimento di Matematica, Piazzale A. Moro 2, I-00185 Roma, Italy

E-mail address: dancona@mat.uniroma1.it 